Contagem incremental de padrões locais em árvores de componentes para cálculo de atributos

\author{
Dennis José da Silva
}

\author{
DisSERTAÇÃO APRESENTADA \\ $\mathrm{AO}$ \\ Instituto DE MATEMÁTiCA E Estatística \\ DA \\ Universidade DE SÃo PaUlo \\ PARA \\ OBTENÇÃO DO TÍTULO \\ $\mathrm{DE}$ \\ Mestre EM CiÊnCIAS \\ Programa: Ciência da Computação \\ Orientador: Prof. Dr. Ronaldo Fumio Hashimoto
}

São Paulo, dezembro de 2017 


\title{
Contagem incremental de padrões locais em árvores de componentes para cálculo de atributos
}

\author{
Esta versão da dissertação contém as correções e alterações sugeridas \\ pela Comissão Julgadora durante a defesa da versão original do trabalho, \\ realizada em 26/10/2017. Uma cópia da versão original está disponível no \\ Instituto de Matemática e Estatística da Universidade de São Paulo.
}

Comissão Julgadora:

- Prof. Dr. Ronaldo Fumio Hashimoto (orientador) - IME-USP

- Prof. Dr. Paulo André Vechiatto de Miranda - IME-USP

- Prof. Dr. Silvio Jamil Ferzoli Guimarães - PUC-MG 


\section{Agradecimentos}

Gostaria de agradecer ao meu pai Aristide, minha mãe Lina, meu irmão Diego e minha cunhada Raquel, por todo o apoio, paciência, incentivo e suporte durante meus estudos.

Aos professores Ismar Frango Silveira, Maria Amelia Eliseo e Luciano Silva da Universidade Presbiteriana Mackenzie, por orientações, conversas e incentivos ao inicio da minha vida acadêmica e pela ajuda em meu ingresso no programa de pós-graduação do IME.

Ao professor Ronaldo Fumio Hashimoto, por ter acreditado em mim e me dado a oportunidade de estudar o curso de pós-graduação do IME. Por ter me introduzido a área de processamento de imagens e pela excelente orientação durante todo curso em especial no desenvolvimento desse trabalho.

Aos meus colegas Wonder Alves e Alexandre Morimitsu, pelas discussões e estudos no desenvolvimento de diversos trabalhos e em especial pelas contribuições neste trabalho.

Aos professores: Paulo André Vechiatto de Miranda e Silvio Jamil Ferzoli Guimarães pelos comentários e sugestões realizados no exame de qualificação.

Aos meus colegas, amigos e familiares um muito obrigado. 


\section{Resumo}

SILVA, D. J. Contagem incremental de padrões locais em árvores de componentes para cálculo de atributos. Dissertação (Mestrado) - Instituto de Matemática e Estatística, Universidade de São Paulo, São Paulo, 2017.

Árvore de componentes é uma representação completa de imagens que utiliza componentes conexos dos conjuntos de níveis de uma imagem e a relação de inclusão entre esses componentes. Essas informações possibilitam diversas aplicações em processamento de imagens e visão computacional, e.g. filtros conexos, segmentação, extração de características entre outras. Aplicações que utilizam árvore de componentes geralmente computam atributos que descrevem os componentes conexos representados pelos nós da árvore. Entre esses atributos estão a área, o perímetro e o número de Euler, que podem ser utilizados diretamente ou indiretamente (para o cálculo de outros atributos). Os bit-quads são padrões de tamanho $2 \times 2$ binários que são agrupados em determinados conjuntos e contados em imagens binárias. Embora o uso de bit-quads resulte em um método rápido para calcular atributos em imagens binárias, o mesmo não ocorre para o cálculo de atributos dos nós de uma árvore de componentes, porque os padrões contados em um nó podem se repetir nos conjuntos de níveis da imagem e serem contados mais de uma vez. A literatura recente propõe uma adaptação dos bit-quads para o cálculo incremental e eficiente do número de buracos na árvore de componentes. Essa adaptação utiliza o fato de cada nó da árvore de componentes representar um único componente conexo e uma das definições do número de Euler para o cálculo do número de buracos. Embora essa adaptação possa calcular o número de Euler, os outros atributos (área e perímetro) não podem ser computados. Neste trabalho é apresentada uma extensão dessa adaptação de bit-quads que permite a contagem de todos os agrupamentos de bit-quads de maneira incremental e eficiente na árvore de componentes. De forma que o método proposto possa calcular todos os atributos que podem ser obtidos pelos bit-quads (além do número de buracos) em imagens binárias na árvore de componentes de maneira incremental.

Palavras-chave: árvore de componentes, bit-quads, atributos incrementais. 


\section{Abstract}

SILVA, D. J. Incremental counting of local patterns in component tree for attribute calculation. Dissertation - Institute of Mathematics and Statistics, University of São Paulo, São Paulo, 2017.

Component tree is a full image representation which uses the connected components of the level sets of the image and these connected components' inclusion relationship. This information can be used in various image processing and computational vision applications, e.g. connected filters, segmentation, feature extraction, among others. In general, applications which uses component trees compute attributes that describe the connected components represented by the tree nodes. Attributes such as area, perimeter and Euler number, can be used directly or indirectly (when they are used to compute other attributes) to describe the component tree nodes in various applications. The bit-quads are binary patterns of size $2 \times 2$ that are grouped in determined sets and counted in binary images to compute area, perimeter (also theirs continuous approximation) and Euler number . Even though the bit-quads usage can yield an efficient method to compute binary image attributes, they cannot be used efficiently to compute attributes for all component tree nodes, since some bit-quads can be counted more than once over the level sets. An adaptation of the bit-quads has been proposed to compute efficiently and incrementally the number of holes for all component tree nodes. This adaptation uses the fact that each component tree node represents a unique connected component and one of Euler number definitions to compute the number of holes. Even though this adaptation can compute Euler number, it cannot compute other attributes derived from the bitquads (area and perimeter). In this work, an extension of this adaptation is proposed to efficiently and incrementally count all bit-quads sets in a component tree. Moreover it yields a method to compute all attributes which can be computed by the bit-quads in binary images in the component tree using an incremental strategy.

Keywords: component tree, bit-quads, incremental attributes. 


\section{Sumário}

$\begin{array}{ll}\text { Lista de Abreviaturas } & \text { ix }\end{array}$

Lista de Símbolos $\quad$ xi

Lista de Figuras $\quad$ xiii

$\begin{array}{lc}\text { Lista de Tabelas } & \text { xvii }\end{array}$

1 Introdução $\quad 1$

1.1 Trabalhos Relacionados . . . . . . . . . . . . . . . . . . . . 4

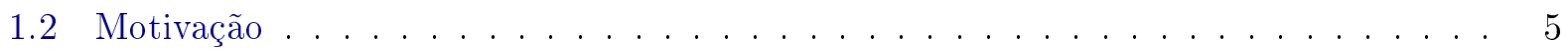

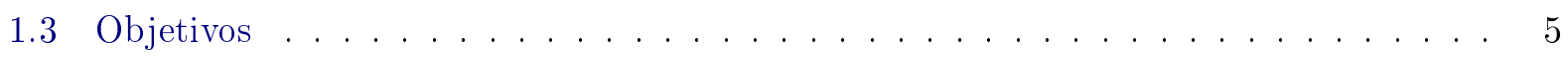

1.4 Organização do Trabalho . . . . . . . . . . . . . . . . . . 5

$\begin{array}{lll}2 & \text { Conceitos } & 7\end{array}$

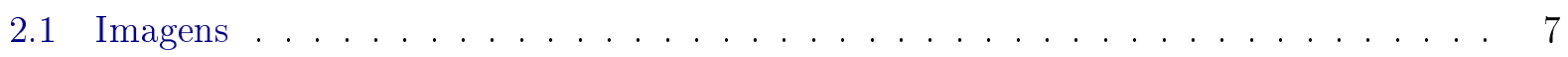

2.1 .1 Imagens como funções $\ldots \ldots \ldots \ldots \ldots \ldots$

$2.1 .2 \quad \operatorname{Grafos} \ldots \ldots \ldots \ldots \ldots \ldots \ldots \ldots$

2.1 .3 Funções de imagem como grafos . . . . . . . . . . . . . . . . . . . 9

2.1 .4 Limiarização e conjunto de níveis . . . . . . . . . . . . . . . . . 11

2.2 Árvore de componentes . . . . . . . . . . . . . . . . . . . . . 13

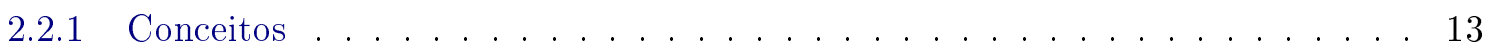

2.2 .2 Computação incremental de atributos . . . . . . . . . . . . . . 16

2.3 Cálculo de atributos utilizando bit-quads . . . . . . . . . . . . . 20

2.3.1 Contagem de bit-quads em imagens binárias . . . . . . . . . . . . . 20

2.3.2 Contagem de número de buracos na árvore de componentes baseada em bit-

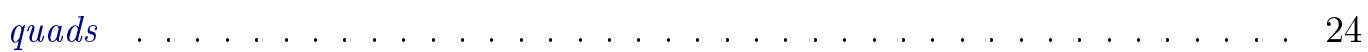

3 Método proposto $\quad 29$

3.1 Definições . . . . . . . . . . . . . . . . . . . . . . . 29

3.1.1 Extensão dos quads para contagem de todos os bit-quads . . . . . . . . . . . 29

3.1.2 Extensão dos padrões de Climent e Oliveira para contagem de todos os quads na árvore de componentes . . . . . . . . . . . . . . . . . . . 31

3.2 Algoritmo incremental para contagem dos padrões propostos . . . . . . . . . . . . 35

3.2 .1 Apresentação . . . . . . . . . . . . . . . . . . . 35

3.2 .2 Corretude . . . . . . . . . . . . . . . . . . . 38 
4 Otimização por Árvore de Decisão $\quad 45$

4.1 Otimização da Função count Utilizando Árvore de Decisão . . . . . . . . . . . . . . . 45

4.2 Implementação da Otimização Utilizando Árvore de Decisão . . . . . . . . . . . . . . . 47

5 Experimentos $\quad 51$

5.1 Análise de Tempo . . . . . . . . . . . . . . . . . . . . . 5 52

5.2 Análise de Precisão . . . . . . . . . . . . . . . . . . . . . . 55

5.3 Conclusão dos Experimentos . . . . . . . . . . . . . . . . . 56

6 Conclusões $\quad 57$

6.1 Trabalhos Futuros . . . . . . . . . . . . . . . . . . 57

$\begin{array}{ll}\text { Referências Bibliográficas } & 59\end{array}$

$\begin{array}{ll}\text { Índice Remissivo } & 61\end{array}$ 


\title{
Lista de Abreviaturas
}

\author{
pixel Elemento de imagem (picture element) \\ ICIP International Conference on Image Processing \\ API Application Programming Interface \\ RGB Red, Green, Blue \\ MI Métodos Incrementais \\ CQNI Contagem de Quads Não Incremental \\ JVM Java Virtual Machine \\ DDR3 Double Data Rate \\ GB Gigabyte \\ $\mathrm{GHz} \quad$ Giga Hertz
}




\section{Lista de Símbolos}

$\mathcal{D}$

$\mathbb{Z}$

$\mathbb{K}$

$\mathcal{P}(X)$

$\Gamma(x)$

$\mathcal{F}(A, B)$

$C R$

$S Q$

$\mathcal{A}_{4}$

$\mathcal{A}_{8}$

$\mathcal{A}$

$\mathcal{C C}(V, \mathcal{A})$

$\mathcal{X}_{\downarrow}^{\lambda}(f)$

$\mathcal{X}_{\lambda}^{\uparrow}(f)$

$\mathcal{L}(f)$

$\mathcal{U}(f)$

$\emptyset$

$T(f)$

$\mathcal{T}(T)$

$\mathcal{S C}(\mathcal{T}, p)$

$\kappa(A)$

$\operatorname{children}(A)$

$n(Q)$

$S_{B}$

$S$

$\mathcal{Q}$

$\mathbb{P}$

$M_{Q_{i}, X}(p)$
Domínio de imagens

Conjunto dos números inteiros

Contra-domínio de imagens

Conjunto potência do conjunto $X$

Vizinhança do vértice $x$

Conjunto de todas as funções de domínio $A$ e contra-domínio $B$

Conjunto cruz

Conjunto quadrado $3 \times 3$

Adjacência de 4-conectividade

Adjacência de 8-conectividade

Adjacência arbitrária (4- ou 8-conectividade)

Conjunto de todos os componentes conexos de $V$ sobre uma adjacência $\mathcal{A}$

Conjunto inferior de valor $\lambda$ da imagem $f$

Conjunto superior de valor $\lambda$ da imagem $f$

Conjunto de todos os componentes conexos dos conjuntos de níveis inferiores da imagem $f$

Conjunto de todos os componentes conexos dos conjuntos de níveis superiores da imagem $f$

Conjunto vazio

Árvore de componentes da imagem $f$

Representação compacta e não redundante da árvore de componentes $T$ Menor componente conexo na árvore representada por $\mathcal{T}$ que contém o pixel $p$

Atributo de um nó $A$ da árvore de componentes

Conjunto de nós filhos de um nó $A$ da árvore de componentes

Contagem de bit-quads do tipo $Q$

Conjunto de símbolos para os quads propostos

Conjunto de símbolos para os padrões propostos

Família dos conjuntos de quads propostos

Família dos conjuntos de padrões propostos

Conjunto matching de quads $Q_{i}$ que ocorreram no pixel $p$ da imagem $X$ 
$\mathcal{M}_{P_{i}, f}(p) \quad$ Conjunto matching de padrões $Q_{i}$ que ocorreram no pixel $p$ da imagem $f$

$\mathcal{W}$ Conjunto de todas as janelas de tamanho $2 \times 2$ de $\mathbb{Z}^{2}$

$\mathcal{W}(A) \quad$ Conjunto de todas as janelas de tamanho $2 \times 2$ que intersectam o nó $A$ da árvore de componentes

$M W_{Q_{i}, A}(W) \quad$ Conjunto matching de quads $Q_{i}$ que ocorreram na janela $W$ de tamanho $2 \times 2$ na imagem $A$

$\mathcal{M} W_{P_{i}, f}(W) \quad$ Conjunto matching de padrões $P_{i}$ que ocorreram na janela $W$ de tamanho $2 \times 2$ na imagem $f$ 


\section{Lista de Figuras}

1.1 Exemplo de poda em uma árvore de componentes utilizando limiar na área (número de pixels de objeto). Neste caso os nós $\mathrm{B}$ e $\mathrm{C}$ são podados porque possuem área menor que 4. Obs.: Os pixels de objeto são os pixels pretos. . . . . . . . . . . . .

1.2 Contagem repetida de bit-quads na árvore de componentes: a) e b): Exemplos de bit-quads; c): árvore de componentes com regiões destacadas que mostram onde os bit-quads são encontrados. O bit-quad em vermelho e o em azul dos nós B e C são repetidos no nó D para o mesmo conjunto de pixels. . . . . . . . . . . . . .

1.3 a) Imagem de entrada convertida em níveis de cinza (imagem retirada da base www. zemris.fer.hr/projects/LicensePlates/english/baza_slika.zip); b) imagem processada pela aplicação de segmentação de placas de veículo. . . . . . . . . . . . . . 3

1.4 a) Imagem de entrada; b) imagem de saída do aplicativo para detecção de aspirinas. 3

1.5 a) Imagem de entrada (imagem retirada da base da competição Born-Digital Images (Web and Email) do ICDAR, disponível em http://rrc.cvc.uab.es/?ch=1\&com= downloads; b) imagem de saída após a poda da max-tree; c) imagem de saída após a poda da min-tree. . . . . . . . . . . . . . . . . . . . . . . .

2.1 a) Imagem em níveis de cinza como função; b) imagem binária como função (os pixels pretos representam pixels de objeto). Os eixos representam as coordenadas $x$ e $y$ no conjunto $\mathcal{D}$ para uma imagem $7 \times 7 \ldots \ldots \ldots \ldots \ldots$

2.2 Representação gráfica de dois grafos. O grafo conexo $G$ e o grafo com dois componentes conexos $H$. Nessa representação os círculos representam vértices e as linhas que ligam dois vértices representam as arestas. Os subconjuntos $\{1,5,2\}$ e $\{2,4\}$ são representados pelas cores: azul e vermelha, respectivamente no grafo $G$. Os subconjuntos $\{d, e\},\{a, b, c\}$ e $\{e, d\}$ são representados pelas cores: azul, vermelha e verde, respectivamente no grafo $H \ldots \ldots \ldots \ldots \ldots \ldots \ldots$

2.3 As imagens exibem as duas adjacências destacadas em vermelho em relação ao pixel do centro. a) imagem com exemplos de adjacência 4-conectividade; b) imagem com exemplos de adjacência 8-conectividade. . . . . . . . . . . . . . . . .

2.4 Exemplo de uma imagem como grafo. a) imagem de exemplo; b) imagem a) como grafo com relação de adjacência 4-conectividade; c) imagem a) como grafo com relação de adjacência 8-conectividade. Os números nos nós representam seu peso (nível de cinza) e a posição de cada vértice representa sua coordenada na grade (domínio) . . . . . . . . . . . . . . . . . . . . . . . . 10 
2.5 Representações de imagens binárias como grafos. a) representação da imagem binária como um grafo utilizando a relação de adjacência de 4-conectividade; b) representação da imagem binária como um grafo com relação de adjacência de 8-conectividade. Os grafos estão inscritos nas imagens.

2.6 Imagem binária representada por grafos com componentes conexos diferentes. Embora as imagens a) e b) sejam as mesmas, os grafos que as representam possuem componentes conexos diferentes. O grafo da imagem a) tem 2 componentes conexos e o grafo da imagem b) é conexo. . . . . . . . . . . . . . . . . .

2.7 Exemplo da operação de limiarização em uma imagem em níveis de cinza por um limiar de valor $5 \ldots \ldots \ldots \ldots \ldots \ldots \ldots \ldots$

2.8 Exemplo de conjuntos de níveis e da propriedade de aninhamento. As imagens da coluna esquerda apresentam os conjuntos de níveis inferiores e as da coluna direita apresentam os conjuntos de níveis superiores. As imagens estão aninhadas em relação à operação de inclusão de baixo para cima. . . . . . . . . . . . . . . . . . . . .

2.9 Exemplo de reconstrução de uma imagem através de seus conjuntos de níveis superiores. Na primeira imagem são marcados os pixels de exemplo no conjunto $\mathcal{X}_{0}^{\uparrow}(f)$. A partir do conjunto $\mathcal{X}_{1}^{\uparrow}(f)$ o pixel vermelho está fora do conjunto, a partir do conjunto $\mathcal{X}_{5}^{\uparrow}(f)$ o pixel verde. No final a imagem em níveis de cinza é reconstruída. . . . . . .

2.10 Exemplos de árvores de componentes com os conjuntos de níveis superiores $\mathcal{U}(f)$ e

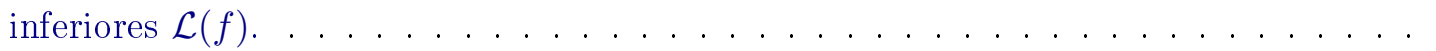

2.11 Exemplo de max-tree e min-tree de uma dada imagem de entrada. Os pixels pretos representam pixels do nó na árvore de componentes que não são armazenados em seu respectivo nó associado, os pixels brancos são pixels do domínio que não pertencem ao nó e os outros pixels são os pixels armazenados nos nós da max-tree ou min-tree. .

2.12 Exemplo do algoritmo que calcula incrementalmente a área de todos os nós de uma árvore de componentes. Nos nós folhas a área é exibida abaixo dos nós, em nós não folha a área é exibida acima do nó, onde o primeiro valor da esquerda para a direita indica a quantidade de pixels na árvore compacta e não redundante (fim do Alg. 2) e os seguintes valores indicam a área de cada nó filho (cada iteração do Alg. 3). Os pixels em vermelho indicam os pixels armazenados na max-tree ou min-tree, os pixels pretos indicam os pixels do nó na árvore de componentes que não são armazenados em seus nós associados e os pixels brancos indicam os pixels do domínio da imagem que não pertencem ao nó.

2.13 Exemplo dos conceitos utilizados por Gray [Gra71] para a definição de imagens binárias no espaço contínuo.

2.14 Duas triangulações para uma imagem binária, onde a região hachurada indica um objeto. Imagem adaptada de [Gra71] . . . . . . . . . . . . . . . . . . . . 21

2.15 Exemplo de triangulação de objeto conexo com buraco. . . . . . . . . . . . . . . . 22

2.16 Bit-quads definidos por Gray. . . . . . . . . . . . . . . . . . . . 22

2.17 Exemplo de uma triangulação de uma imagem binária e a utilização de bit-quads para contar vértices, arestas e regiões. Os quadrados hachurados indicam pixels de objeto e o exemplo $S Q .5$ é referente ao um bit-quad $Q D$. Imagem retirada de [Gra71]. 23 
2.18 Representação de um bit-quad de $Q D$ em uma adjacência de 8-conectividade com 8 vértices e em uma adjacência de 4-conectividade com 8 vértices. . . . . . . . . . . 24

2.19 Exemplo que demonstra padrões que precisam utilizar pixels do tipo $G$ para não obter contagem incorreta. Imagem retirada de $[\mathrm{CO} 14] \ldots \ldots \ldots$

2.20 Exemplo da utilização de soma para juntar as contagens de quads dos nós filhos com o nó pai. Na imagem a), o quad de $Q 1$ contando nó $C$ não existe em seu nó pai (nó $B$ ). Na imagem b), a soma das contagens é o suficiente para juntar as mesmas. Imagem retirada de $[\mathrm{CO} 14] . \ldots \ldots \ldots \ldots \ldots$. . . . . . . . . . . . . . . . . . . .

2.21 Exemplos de quads nos nós de uma árvore de componentes. Imagens retiradas de

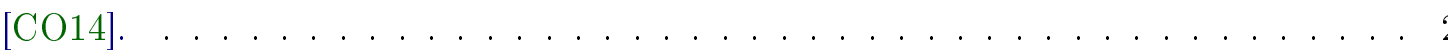

3.1 Exemplo de translações dos quads $Q$ (em vermelho) e $Q^{\prime}$ (em azul) e as comparações feitas nas regiões da imagem definidas pelos domínios dos quads. . . . . . . . . . 30

3.2 Domínios dos quads contados em uma imagem binária. Os domínios dos quads de $Q_{1}$ são exibidos na imagem a), os domínios dos quads de $Q_{2}$ são exibidos na imagem b)

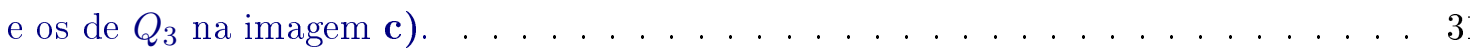

3.3 Translações de dois padrões $P$ e $P^{\prime}$ por um pixel $p$ e a verificação de pixels nas vizinhanças definidas pelos domínios dos padrões transladados. . . . . . . . . . . 32

3.4 Exemplo de transformação de padrões na contagem de quads. O padrão $P$ conta um quad do tipo $Q_{3}$ no nó $\hat{A}$ da árvore (quando o pixel $p$ é analisado), no nó $\hat{D}$ ao analisar o pixel q, o padrão $P^{\prime}$ é contado como um quad do tipo $Q_{4}$ para o mesmo conjunto de pixels (marcado como um retângulo vermelho) em que o padrão $P$ que representa um quad do tipo $Q_{3}$ havia sido contado, causando assim uma transformação de padrões. O padrão $P^{\prime \prime}$ que representada um quad do tipo $Q_{2}$ é contado no nó $\hat{A}$ e esse padrão/quad continua existindo no nó $\hat{D}$, neste caso não houve transformação de padrões.

3.5 Contagem de padrões utilizando o algoritmo incremental. Os números em verde indicam a contagem realizada pela função Pre-Process, os números em vermelho indicam a contagens acumuladas dos padrões nos nós filhos realizadas pelo procedimento MERGE após todas as iterações do laço do algoritmo, os números em azul indicam a remoção dos quads transformados realizada pelo procedimento Post-Process. Os procedimentos que realizam operações com o valor 0 foram suprimidos. . . . . . . . . 37

3.6 Exemplos de duas janelas formadas por translações dos domínios dos quads $Q$ e $Q^{\prime}$. A janela $W$ é formada por $D_{Q}+p$ (em azul) e a janela $W^{\prime}$ é formada por $D_{Q^{\prime}}+p$

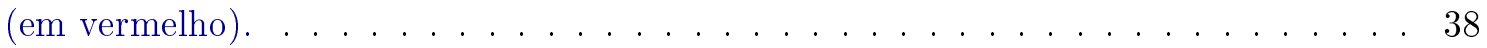

3.7 Janelas em uma imagem em nível de cinza definidas por translação dos domínios dos padrões $P$ (em azul) e $P^{\prime}$ (em vermelho) . . . . . . . . . . . . . . . 39

4.1 Exemplo de árvore de decisão para classificação de um triângulo em equilátero, isósceles ou escaleno. As arestas em verde na árvore de decisão mostram o caminho percorrido para o triângulo $A B C \ldots \ldots \ldots \ldots \ldots$

4.2 Pixels vizinhos de um pixel $p$ em uma vizinhança de 8-conectividade. . . . . . . . . 46 
4.3 Árvore de decisão resumida para a função count. Cada vértice representa um pixel vizinho de $p$, cada aresta representa a relação de ordem de $p$ com o vizinho e as folhas representam funções $n p$ onde cada valor é a quantidade de padrões de determinado tipo $\left(P_{1}, P_{2}, P_{3}, P_{4}, P_{D}, P_{1 T}, P_{2 T}, P_{3 T}, P_{D T}\right.$ respectivamente de cima para baixo na figura) contados por count. . . . . . . . . . . . . 46

4.4 Exemplo de codificação e indexação de uma vizinhança na matriz da árvore de decisão da função count. . . . . . . . . . . . . . . . . . . . . 47

4.5 imagem $i m g$ gerada no Alg. $12 \ldots \ldots \ldots \ldots$. . . . . . . . . . . . . 48

5.1 Gráfico comparativo de tempo entre o algoritmo proposto e sua otimização. . . . . . 52

5.2 Gráfico comparativo de tempo do algoritmo proposto otimizado e MI. . . . . . . . . 53

5.3 Gráfico comparativo de tempo do algoritmo proposto otimizado e o algoritmo de chain code. . . . . . . . . . . . . . . . . . . . 53

5.4 Gráfico comparativo de tempo do algoritmo proposto otimizado e o algoritmo ingênuo de contagem de quads. . . . . . . . . . . . . . . . . . . . 54 54

5.5 Imagem utilizada para a análise de precisão. . . . . . . . . . . . . 55 


\section{Lista de Tabelas}

2.1 Tabela com a contribuição de cada bit-quad para o número de Euler. . . . . . . . . . 23

5.1 speedup do algoritmo proposto otimizado em relação aos outros métodos analisados. . 54

5.2 Erros de área, perímetro e circularidade entre os MI e o algoritmo proposto. . . . . . 56

5.3 Erro no cálculo de perímetro do algoritmo de chain code e do algoritmo proposto. . . 56 
xviii LISTA DE TABELAS 


\section{Capítulo 1}

\section{Introdução}

Imagens podem ser representadas em diversas formas como: (i) conjuntos [DL03], (ii) funções [DL03], (iii) matrizes [Mat16], (iv) grafos [FSL04] entre outras representações. Geralmente formulações teóricas representam imagens como funções que levam um pixel (picture element) a um valor numérico que representa sua intensidade. APIs (Application Programming Interface) e bibliotecas para processamento de imagens em computadores normalmente representam imagens como matrizes [Mat16, com16, Ope15], onde cada elemento da matriz representa um nível de cinza (representação geralmente utilizada para imagens que possuem apenas uma cor). Em Morfologia Matemática a representação por árvore de componentes de uma imagem em níveis de cinza tem ganhado destaque em meio acadêmico com aplicações em segmentação [TSR $\left.{ }^{+} 15\right]$, processamento de imagens [SW09], reconhecimento de padrões [BGL ${ }^{+}$07], entre outras. Uma árvore de componentes pode ser construída a partir de uma imagem representada como matriz em tempo quasi-linear no número de pixels da imagem e a representação de matriz pode ser reconstruída eficientemente a partir da árvore de componentes [NC06]. Além disso, as árvores de componentes possuem propriedades interessantes para criação de filtros conexos e para caracterização de componentes conexos de vários limiares da imagem, tornando-se assim uma representação interessante para processamento de imagens em computadores [SW09].

Uma árvore de componentes é formada por nós que representam componentes conexos presentes nos conjuntos de níveis de uma imagem e sua relação de parentesco é dada pela relação de inclusão desses componentes. Na prática esses nós não apenas representam os componentes conexos em diversos limiares, mas também são utilizados para armazenar atributos, e.g. área, volume, perímetro, momento, entre outros. Esses atributos podem ser utilizados para caracterização da imagem e a criação de filtros conexos. Geralmente os atributos são utilizados para selecionar um conjunto de nós da árvore por meio de um valor de limiar. Quando o atributo é crescente uma operação de poda (i.e. dado um nó selecionado, todos os seus descendentes também são selecionados e o mesmos são removidos) e a reconstrução da imagem a partir da árvore podada é suficiente para criação de um filtro conexo (abertura ou fechamento por atributo). Quando o atributo não é crescente, uma estratégia mais elaborada como as propostas por Salembier e Wilkison [SW09] ou o método subtrativo proposto por Urbach e colaboradores [URW07] deve ser utilizada. A Fig. 1.1 exibe um exemplo de filtro conexo utilizando operação de poda e reconstrução em uma árvore de componentes. 


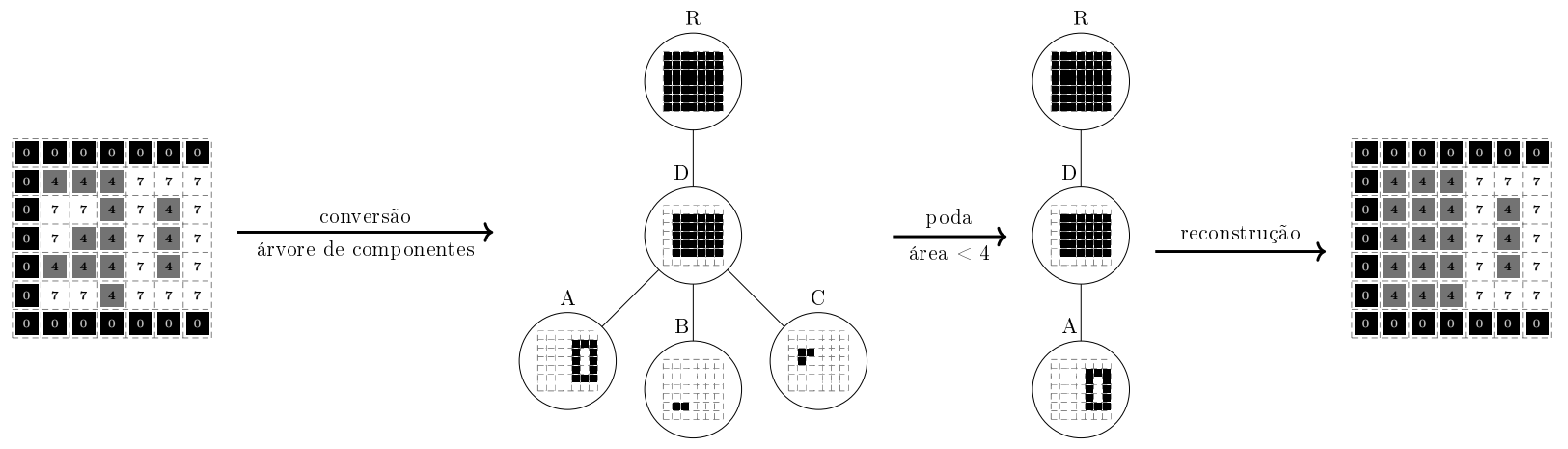

Fig. 1.1: Exemplo de poda em uma árvore de componentes utilizando limiar na área (número de pixels de objeto). Neste caso os nós B e C são podados porque possuem área menor que 4. Obs.: Os pixels de objeto são os pixels pretos.

Os atributos dos nós de uma árvore de componentes geralmente são obtidos por cálculos que utilizam informações do componente conexo representado pelo nó, por exemplo, o próprio conjunto de pixels, relação de inclusão entre os nós e intensidade dos pixels do nó na imagem original podem ser utilizados para calcular os seguintes atributos: altura, profundidade, volume, entre outros. Considerando que os componentes conexos representados pelos nós da árvore de componentes são imagens binárias, os atributos calculáveis em imagens binárias podem ser utilizados para descrever esses nós, e.g. área, perímetro, momento, número de Euler, etc. Em particular, Gray [Gra71] criou um algoritmo que calcula área, perímetro e número de Euler eficientemente por meio da contagem de padrões binários (chamados de bit-quads) contidos em uma imagem binária. Apesar do algoritmo de Gray ser eficiente para cálculo de atributos em uma imagem binária, a sua utilização no cálculo de todos os nós de uma árvore de componentes em geral é ineficiente porque o algoritmo contaria repetidamente padrões que estejam contidos em um conjunto de pixels de um componente conexo que se repete diversas vezes nos conjuntos de níveis (diversos nós da árvore de componentes). A Fig. 1.2 exibe essa repetição.

a)

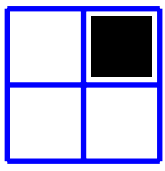

b)

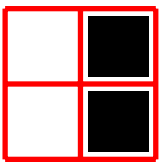

c)

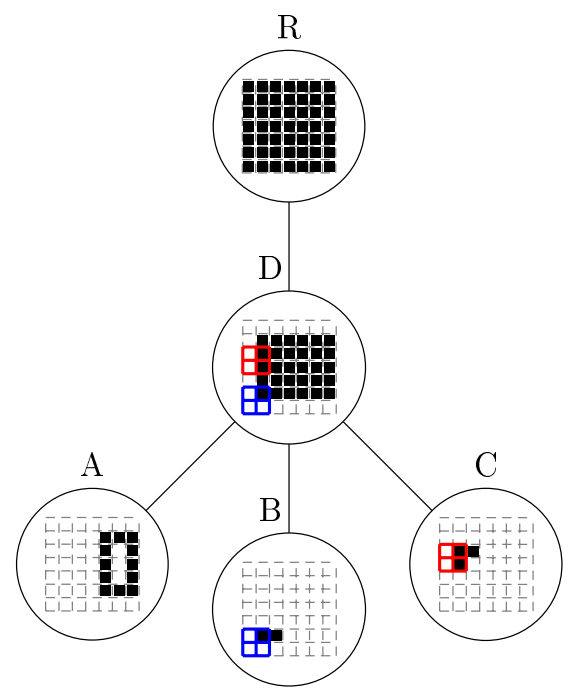

Fig. 1.2: Contagem repetida de bit-quads na árvore de componentes:

a) e b): Exemplos de bit-quads; c): árvore de componentes com regiões destacadas que mostram onde os bit-quads são encontrados. O bit-quad em vermelho e o em azul dos nós $B$ e $C$ são repetidos no nó $D$ para o mesmo conjunto de pixels.

Climent e Oliveira [CO14], se basearam nos padrões binários de Gray e elaboraram conjuntos de padrões para serem utilizados de maneira eficiente na árvore de componentes, possibilitando assim o cálculo do número de buracos em todos os nós da árvore. Esses conjuntos utilizam comparações de níveis de cinza para contagem dos padrões necessários para o cálculo do número de Euler e a 
extração do número de buracos. Esse método foi utilizado para segmentação de placa de veículos por meio de poda por número de buracos na árvore de componentes. Apesar do método eficiente proposto por Climent e Oliveira, a contagem dos padrões originais de Gray não são calculados, impossibilitando o cálculo de outros atributos extraídos pelos mesmos, e.g. área, perímetro e suas aproximações contínuas. Uma extensão dos padrões de Climent e Oliveira é proposta por Silva et. al ([SAMH16]) para contagem dos padrões de Gray na árvore de componentes. Esses padrões possibilitaram calcular aproximações contínuas da área e o perímetro que são utilizadas para o cálculo da circularidade e a segmentação de objetos circulares em uma imagem em níveis de cinza por meio de ultimate attribute opening [AMH15, HM11].

O método proposto em [SAMH16] possui 60 padrões e cada padrão contem 3 símbolos comparáveis, portanto, apesar de gastar tempo linear no número de pixels o método realiza 180 comparações para cada pixel da imagem. Nesse trabalho é apresentada uma otimização para esse algoritmo que diminui para 16 o número de comparações por pixel. A melhora de desempenho é apresentada de maneira experimental, assim como a comparação entre outros métodos da literatura para o cálculo dos mesmos atributos.

Com a implementação do método apresentado neste trabalho, foram reproduzidas as aplicações apresentadas em [CO14] (segmentação de placas de veículos), [SAMH16] (segmentação de aspirinas), [LMG05] (localização de texto). O aplicativo da segmentação de placas de veículo utiliza uma abertura por área na imagem (poda por área na árvore de componentes) para remover pequenos buracos. Depois, os nós que tiverem exatamente oito ou nove buracos são reconstruídos com o nível de cinza armazenado no nó. A Fig. 1.3 mostra um exemplo de segmentação utilizando esses procedimentos.

a)

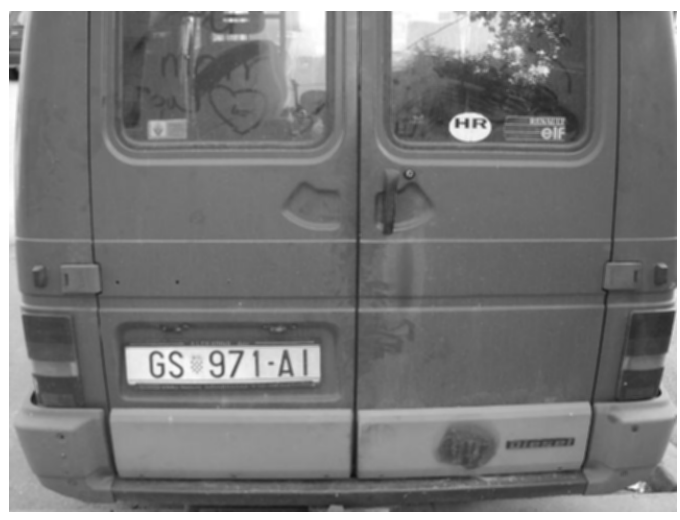

b)

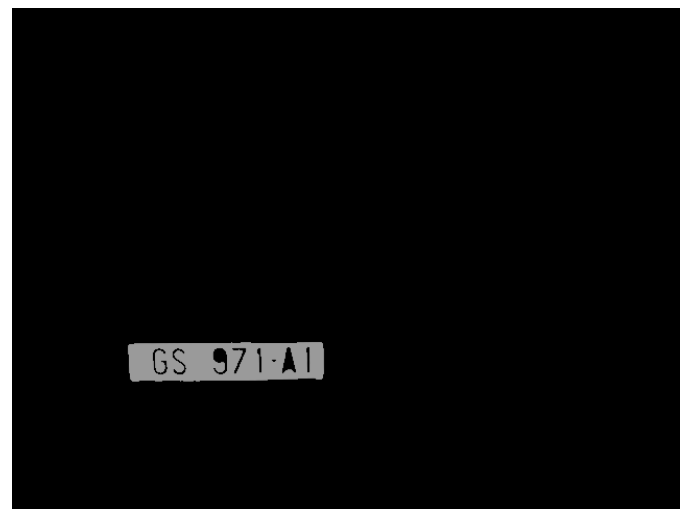

Fig. 1.3: a) Imagem de entrada convertida em niveis de cinza (imagem retirada da base www.zemris.fer. $\mathrm{hr} /$ projects/LicensePlates/english/baza_slika.zip); b) imagem processada pela aplicação de segmentação de placas de veículo.

A aplicação que resultou nas imagens exibidas na Fig. 1.3 utilizou os atributos área e número de buracos obtidos pelo método proposto neste trabalho.

O aplicativo para segmentação de aspirinas, utiliza o método ultimate attribute opening em uma árvore de componentes com os nós que tenham circularidade maior que 0,92 e área maior que 500 pixels selecionados. Então uma imagem de resíduo ([AMCH13]) é gerada e utilizada para a extração das aspirinas conforme exibido na Fig. 1.4.

a)

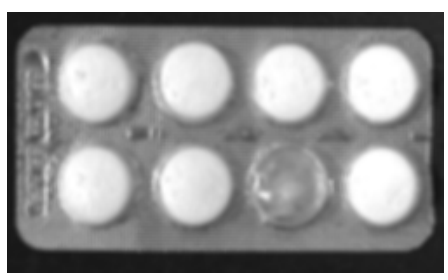

b)

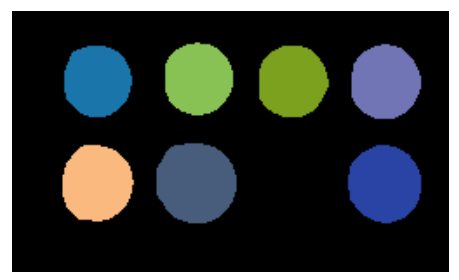

Fig. 1.4: a) Imagem de entrada; b) imagem de saída do aplicativo para detecção de aspirinas. 
Nesse aplicativo, foram utilizados os atributos: área $a$, aproximação contínua de perímetro $\hat{p}$ e aproximação contínua de área $\hat{a}$, calculados pelo método proposto. Os atributos $\hat{p}$ e $\hat{a}$ foram utilizados para forma o atributo da circularidade $c=\frac{4 \pi \hat{a}}{\hat{p}^{2}}$ que junto com a área foram responsáveis por selecionar os nós para a utilização do ultimate attribute opening.

No aplicativo de localização de texto foram utilizados os atributos complexidade e compacidade para realização de podas na max-tree e min-tree(veja Sec. 2.2.1) utilizando o algoritmo de Viterbi [SG00]. O resultado obtido é apresentado na Fig.1.5.

a)

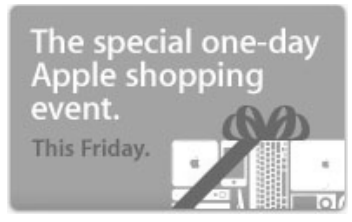

b)

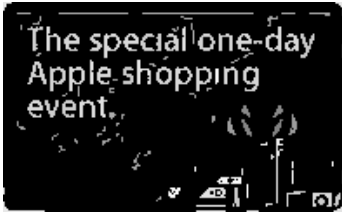

c)

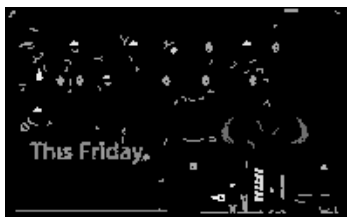

Fig. 1.5: a) Imagem de entrada (imagem retirada da base da competição Born-Digital Images (Web and Email) do ICDAR, disponivel em http://rrc.cvc.uab.es/?ch=186com=downloads; $\boldsymbol{b}$ ) imagem de saída após a poda da max-tree; c) imagem de saída após a poda da min-tree.

Neste exemplo de aplicação, os atributos: área $a$ e perímetro $p$ foram calculados com o algoritmo proposto e utilizados para computar a complexidade $c x=\frac{p}{a}$ e a compacidade $c c=\frac{a}{p^{2}}$ dos nós das árvores de componentes. Como esses atributos não são crescentes a poda foi realizada pelo algoritmo de Viterbi conforme exposto em [LMG05].

\subsection{Trabalhos Relacionados}

Diversos atributos podem ser utilizados para caracterização de imagens, entre eles a área, perímetro e o número de Euler podem ser calculados por conjuntos de padrões binários (bit-quads) definidos por Gray. Com a composição desses atributos é possível obter outros atributos como alongamento médio, comprimento de linha, largura média de linha, entre outros [Gra71].

Os atributos que descrevem componentes conexos podem ser utilizados em operadores morfológicos. Operadores de abertura e fechamento por atributo (para atributos crescentes) e thinnings por atributo (para atributos não-crescentes) são apresentados em [BJ96]. Esses operadores utilizam atributos calculados nos componentes conexos e eliminam aqueles componentes que não satisfazem um critério pré-definido. Esse critério normalmente é um limiar dos atributos, por exemplo, o operador de abertura por área, proposto por Vicent [Vin93] que remove os componentes conexos de uma imagem binária com área menor que um limiar pré-definido. Uma extensão desses operadores para imagens em níveis de cinza é obtida através da aplicação do operador em cada imagem binária dos conjuntos de níveis dessa imagem seguida pela operação de reconstrução.

Os operadores morfológicos para imagens em níveis de cinza podem ser facilmente obtidos quando a imagem está representada como uma árvore de componentes através de uma operação de poda e reconstrução [SW09]. Desta forma se faz necessário o cálculo eficiente de atributos para cada nó da árvore. Muitos atributos já possuem algoritmos para o cálculo eficiente, por exemplo, área, altura e volume [NC06]. Em particular existe um algoritmo proposto por Climent e Oliveira [CO14] baseado em bit-quads que calcula o número de buracos eficientemente para cada nó de uma árvore de componentes, porém, esse algoritmo não é flexível o bastante para calcular os outros atributos calculáveis pelos bit-quads definidos por Gray. Uma extensão desse algoritmo é proposta em [SAMH16] para o cálculo eficiente de todos os atributos que podem ser calculados com os bit-quads na árvore de componentes. O texto desta dissertação apresenta os detalhes dessa extensão, com análise de desempenho, prova de corretude, experimentos e otimização. 


\subsection{Motivação}

Após a reprodução dos resultados obtidos por Climent e Oliveira em [CO14] surge a dúvida se seria possível também calcular o perímetro utilizando a contagem de bit-quads na árvore de componentes (os padrões de Climent e Oliveira calculam apenas o número de buracos/número de Euler). A partir do desenvolvimento dos padrões para o cálculo do perímetro surge a ideia de estender os conjuntos de padrões para calcular todos os bit-quads propostos por Gray. O resultado dessa extensão foi publicado como um artigo no ICIP 2016 (IEEE International Conference on Image Processing) e é descrito em detalhes por esse documento.

Apesar do método proposto em [SAMH16] gastar tempo linear no número de pixels, resultados experimentais mostraram que o tempo gasto para calcular os atributos era maior que o tempo gasto por uma adaptação dos métodos conhecidos na literatura para calcular o mesmo conjunto de atributos. Esses resultados motivaram o desenvolvimento de uma otimização do algoritmo proposto que conforme será apresentado na Sec. 5 foi mais rápido que todos os métodos executados nos experimentos.

\subsection{Objetivos}

O objetivo deste trabalho é o desenvolvimento do algoritmo proposto em [SAMH16] para contagem de bit-quads na árvore de componentes de maneira incremental e eficiente. Neste sentido o objetivo do trabalho pode ser dividido nos seguintes subobjetivos:

- Aprimoramento e otimização (realizada utilizando árvore de decisão) da função de contagem de padrões.

- Corretude do algoritmo proposto (Prova do Teo. 1, enunciado na Sec. 3.2.2).

- Verificação da eficiência do algoritmo e sua otimização, através da comparação com métodos conhecidos na literatura para o cálculo dos atributos calculáveis pelo algoritmo proposto neste trabalho.

O objetivo desse método é disponibilizar um algoritmo eficiente que calcule os atributos propostos, permitindo assim o desenvolvimento de novos trabalhos e o aprimoramento de trabalhos já existentes que utilizam esses atributos. Exemplos de trabalhos que utilizam os atributos que podem ser calculados pelo método proposto são encontrados em [CO14, LMG05].

\subsection{Organização do Trabalho}

Este trabalho está dividido em 6 capítulos, consistindo da introdução ao trabalho apresentada no Capítulo 1. No Capítulo 2 são apresentados os conceitos que são utilizados no Capítulo 3, onde é descrito o desenvolvimento do algoritmo e sua corretude. O Capítulo 4 expõe a otimização do algoritmo utilizando uma árvore de decisão. No Capítulo 5 são apresentados resultados experimentais analisando o tempo gasto e a precisão da implementação em software do método proposto e o trabalho se encerra com a conclusão e propostas para a extensão do trabalho no Capítulo 6. 


\section{Capítulo 2}

\section{Conceitos}

Neste capítulo serão apresentados os conceitos utilizados ao longo do trabalho com ênfase na árvore de componentes. Considerando que uma árvore de componentes pode ser construída a partir de qualquer conjunto associado a outro conjunto finito que possua ordem total e uma relação de adjacência, grafo se apresenta uma estrutura adequada para representar imagens nesse sentido. Por outro lado, funções são representações que melhor descrevem os conceitos de conjunto de níveis que é utilizado para a montagem da árvore de componentes. Neste capítulo, ambas representações serão definidas, associadas e utilizadas de acordo com os assuntos abordados nas seções seguintes.

\subsection{Imagens}

\subsubsection{Imagens como funções}

Uma imagem pode ser representada como uma função $f$ que mapeia uma grade retangular finita $\mathcal{D}$ a um valor inteiro, ou seja, $f: \mathcal{D} \rightarrow \mathbb{K}$, tal que $\mathcal{D} \subset \mathbb{Z}^{2}$ e $\mathbb{K}=\{0,1,2, \ldots, K\}$. Se $\mathbb{K}=\{0,1\}$, então a imagem é binária e a mesma pode ser representada por um conjunto $X$ definido como $X=\{p \in \mathcal{D}: f(p)=1\}$. Essas representações são alternadas no texto conforme a conveniência do contexto. Uma imagem multibanda é representada por um conjunto de funções tais que cada função está associada a uma banda, por exemplo, uma imagem colorida RGB (Red, Green, Blue) que geralmente possui três bandas: uma para cor vermelha, outra para a verde e outra para azul, pode ser representada por três funções $f^{R}, f^{G}, f^{B}$, onde cada imagem (função) representa uma banda.

Um elemento do domínio da imagem $p \in \mathcal{D}$ é chamado de pixel (picture element) e o mesmo representa uma posição na grade (domínio) por um par ordenado $(x, y)$, onde $x$ representa uma coluna da grade e y uma linha. A Fig. 2.1 exibe um exemplo de imagem em níveis de cinza e uma imagem binária como funções.

a)

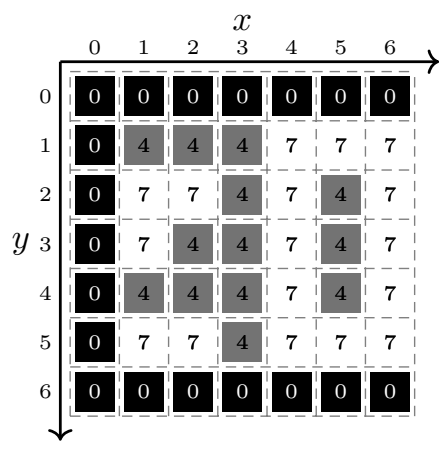

b)

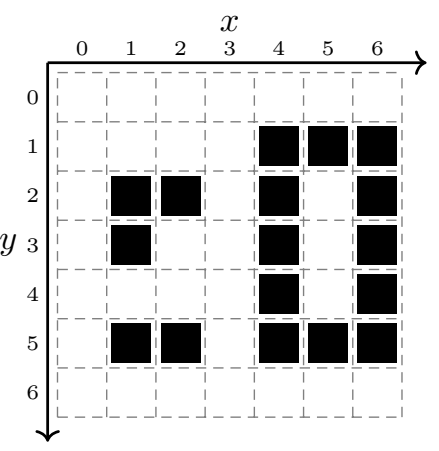

Fig. 2.1: a) Imagem em niveis de cinza como função; b) imagem binária como função (os pixels pretos representam pixels de objeto). Os eixos representam as coordenadas $x$ e y no conjunto $\mathcal{D}$ para uma imagem $7 \times 7$. 
Na Fig. 2.1, o conjunto $\mathcal{D}=\{0,1,2,3,4,5,6\}^{2}$ em ambas as imagens e os pixels são representados por coordenadas $(x, y)$. Na imagem em níveis de cinza a aplicação da função resulta em um nível de cinza e na imagem binária a aplicação da função indica se o pixel é branco (valor 0) ou preto (valor 1), por exemplo, seja $f$ a imagem de níveis de cinza e $g$ a imagem binária na Fig. 2.1, então $f(4,1)=7, f(2,3)=4, f(0,0)=0, g(4,1)=1, g(2,4)=0$ e assim por diante. A imagem $g$ também pode ser representada pelo conjunto $X=\{(1,2),(2,2),(1,3),(1,5),(2,5),(4,1),(5,1),(6,1)$, $(6,2),(6,3),(6,4),(6,5),(5,5),(4,5),(4,4),(4,3),(4,2)\}$.

\subsubsection{Grafos}

Um grafo $G$ é definido por um par $(V, E)$, tal que $V$ é um conjunto finito qualquer de vértices (pontos) e $E$ é uma relação binária, irreflexiva e simétrica em $V$, ou seja, $E \subseteq V \times V,(x, x) \notin E$ e $(x, y) \in E \Leftrightarrow(y, x) \in E$. Os elementos do conjunto $V$ são chamados de vértices e os elementos do conjunto $E$ de arestas. Todos os subconjuntos de um conjunto qualquer $X$ forma o conjunto potência e é denotado por $\mathcal{P}(X)$, por exemplo, $\mathcal{P}(V)$ são todos os subconjuntos de vértices de um grafo $G=(V, E)$. O mapeamento $\Gamma: V \rightarrow \mathcal{P}(V)$ onde para todo $x \in V, \Gamma(x)=\{y \in V:(x, y) \in E\}$ é chamado de vizinhança de $x$. Se $y \in \Gamma(x)$ então $y$ é vizinho de $x$ e $x$ e $y$ são adjacentes.

Seja $G=(V, E)$ um grafo, $X \subseteq V$ um subconjunto de vértices e $x_{0}, x_{n} \in X$ dois vértices em $X$, um caminho de $x_{0}$ a $x_{n}$ é uma sequência de vértices sem repetição $\pi=\left(x_{0}, x_{1}, \ldots, x_{n}\right)$ tal que para qualquer $i \in\{0,1, \ldots, n-1\}, x_{i}, x_{i+1} \in X$ e $x_{i+1} \in \Gamma\left(x_{i}\right)$. O comprimento de um caminho é a quantidade de arestas do caminho e a distância entre dois vértices é o comprimento do caminho mais curto entre esses vértices. Dois vértices $x, y \in X$ estão conectados em $X$ se existe um caminho de $x$ a $y$ em $X$. O conjunto $X$ é chamado de conexo se para todo par de vértices $x, y \in X, x$ e $y$ são conectados em $X$. Um conjunto $Y \subseteq V$ é um componente conexo de $X$ se $Y \subseteq X$, é conexo e maximal, ou seja, se $Y$ é um componente conexo de $X$ e existe um conjunto $Z$ conexo tal que $Y \subseteq Z \subseteq X$ então $Y=Z$.

Um grafo $G=(V, E)$ é chamado de grafo conexo se o conjunto de vértices $V$ possui exatamente um componente conexo. A Fig. 2.2 exibe dois grafos, um conexo e outro não conexo.
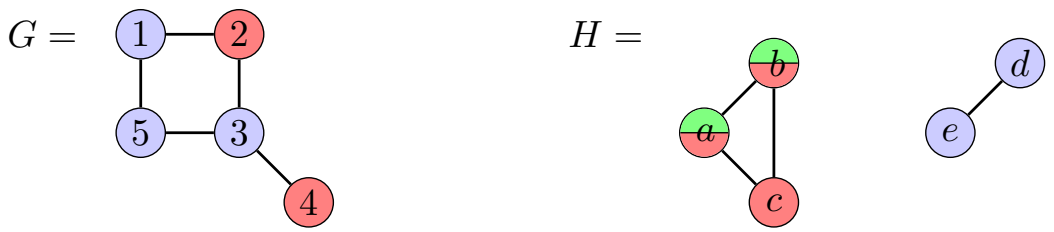

Fig. 2.2: Representação gráfica de dois grafos. O grafo conexo $G$ e o grafo com dois componentes conexos $H$. Nessa representação os círculos representam vértices e as linhas que ligam dois vértices representam as arestas. Os subconjuntos $\{1,5,2\}$ e $\{2,4\}$ são representados pelas cores: azul e vermelha, respectivamente no grafo $G$. Os subconjuntos $\{d, e\},\{a, b, c\}$ e $\{e, d\}$ são representados pelas cores: azul, vermelha e verde, respectivamente no grafo $H$.

Os grafos da Fig. 2.2 são definidos por seus conjuntos de vértices e arestas da seguinte maneira: $G=(V, E), H=\left(V^{\prime}, E^{\prime}\right)$, onde $V=\{1,2,3,4,5\}, E=\{(1,2),(1,5),(2,3),(3,5),(3,4)\}, V^{\prime}=$ $\{a, b, c, d, e\}$ e $E^{\prime}=\{(a, b),(a, c),(b, c),(d, e)\}$. No grafo $G$ os vértices podem ser separados em dois conjuntos: os de cor azul $X=\{1,5,3\}$ e os de cor vermelha $Y=\{2,4\}$. Neste caso, o conjunto $X$ é conexo já que todos pares de vértices são conectados entre si pelos seguintes caminhos: $\pi_{1,2}=(1,2)$, $\pi_{1,5}=(1,5), \pi_{5,2}=(5,1,2)$ em $X$. O conjunto $Y$ não é conexo já que não existe caminho em $Y$ que conecte 3 e 4 . No grafo $H$ os vértices podem ser separados em três conjuntos os que contém vermelho $X^{\prime}=\{a, b, c\}$, os de cor azul $Y^{\prime}=\{e, d\}$ e os que contém verde $Z^{\prime}=\{a, b\}$. Neste caso, $H$ não é conexo e possui dois componentes definidos pelos conjuntos $X^{\prime}$ e $Y^{\prime}$. O conjunto $Z^{\prime}$ é conexo, mas não é um componente já que não é maximal, isso pode ser verificado pela seguinte relação: $Z^{\prime} \subseteq X^{\prime} \subseteq V^{\prime}$ e $Z^{\prime} \neq X^{\prime}$, ou seja, o conjunto $Z^{\prime}$ pode aumentar e ainda assim continuar conexo, portanto, não é um componente conexo. 


\subsubsection{Funções de imagem como grafos}

O conjunto de todas as funções que mapeiam um conjunto $A$ a um conjunto $B$ é denotado por $\mathcal{F}(A, B)$ ou simplesmente por $\mathcal{F}$ quando os conjuntos $A$ e $B$ estão implícitos (e.g. o conjunto de todas as imagens pode ser representado apenas por $\mathcal{F}$, já que por definição $A=\mathcal{D}$ e $B=\mathbb{K})$. Seja $G=(V, E)$ um grafo e $\mathcal{F}(V, W)$ todas as funções que mapeiam vértices de $G$ a um conjunto de pesos $W$, tal que $W$ é um conjunto finito com ordem total (por exemplo, um subconjunto finito de números racionais ou inteiros). Dada uma função $F \in \mathcal{F}(V, W)$, a tripla $(V, E, F)$ é chamada de grafo com pesos nos vértices. Dado um vértice $v \in V$, a aplicação da função $F(v)$ resulta em um peso ou nivel.

Para representar uma imagem $f: \mathcal{D} \rightarrow \mathbb{K}$ como um grafo, é definido uma relação de adjacência entre os pixels, mesmo que a literatura possua aplicações e definições de adjacência gerais [MAH15, SW09], o método proposto nesse trabalho considera apenas as duas relações de adjacência mais comuns para imagens 2D: a relação de adjacência 8-conectividade e 4-conectividade.

A adjacência 4-conectividade $\mathcal{A}_{4}$ é uma relação entre os pixels que associa cada pixel a quatro outros pixels posicionados a cima, a direita, a esquerda e a baixo na grade que define o domínio da imagem. Seja $C R=\{(-1,0),(0,1),(1,0),(0,-1)\}$ o conjunto cruz, então uma dupla de pixels $p, q \in \mathcal{D}$ está em $\mathcal{A}_{4}$ se e somente se $\exists r \in C R$, tal que, $q=p+r$. Dessa relação de adjacência e de um pixel $p \in \mathcal{D}$, define-se o conjunto $\mathcal{A}_{4}(p)=\{r+p: r \in C R\}$ como o conjunto de todos os pixels adjacentes a $p$ por uma relação de adjacência de 4-conectividade. Similarmente a adjacência 8-conectividade $\mathcal{A}_{8}$ é uma relação entre os pixels que associa cada pixel aos 4 pixels em $\mathcal{A}_{4}$ mais os 4 pixels posicionados a suas diagonais. Seja o conjunto $S Q=C R \cup\{(-1,-1),(1,-1),(1,1),(-1,1)\}$ o conjunto quadrado $3 \times 3$, então uma dupla de pixels $(p, q) \in \mathcal{D} \times \mathcal{D}$ está em $\mathcal{A}_{8}$, se e somente se $\exists r \in S Q$, tal que, $q=r+p$. O conjunto de todos os pixels adjacentes a um pixel $p \in \mathcal{D}$ pela relação de adjacência 8-conectividade é denotado por $\mathcal{A}_{8}(p)=\{p+r: r \in S Q\}$. Quando a conectividade de uma relação de adjacência não precisa ser definida, a relação de adjacência é denotada por $\mathcal{A}$ e o conjunto de pixels adjacentes a um dado pixel $p \in \mathcal{D}$ é denotado por $\mathcal{A}(p)$. Neste caso, $\mathcal{A}=\mathcal{A}_{4}$ ou $\mathcal{A}=\mathcal{A}_{8}$ e qualquer resultado ou afirmação sobre $\mathcal{A}$ vale para ambas adjacências $\mathcal{A}_{4}$ e $\mathcal{A}_{8}$. As relações de adjacência são ilustradas com um exemplo na Fig. 2.3.

a)

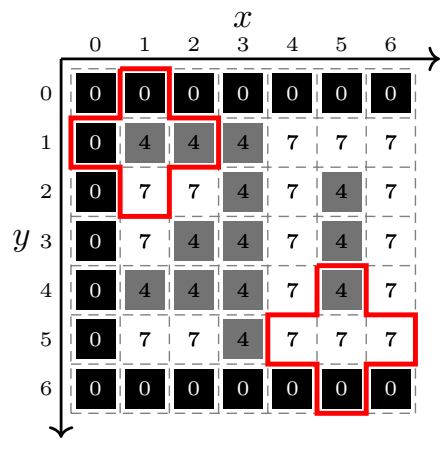

b)

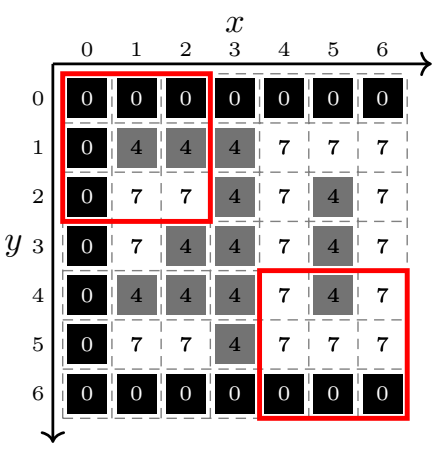

Fig. 2.3: As imagens exibem as duas adjacências destacadas em vermelho em relação ao pixel do centro. a) imagem com exemplos de adjacência 4-conectividade; b) imagem com exemplos de adjacência 8conectividade.

As imagens da Fig. 2.3 exibem exemplos das relações de adjacência. Na imagem a) os pixels $p=(1,1)$ e $q=(5,5)$ possuem uma relação de adjacência 4-conectividade, o conjunto dos $p i$ xels adjacentes desses pixels são $\mathcal{A}_{4}(p)=\{(0,1),(1,0),(2,1),(1,2)\}$ e $\mathcal{A}_{4}(q)=\{(4,5),(5,4),(6,5)$, $(5,6)\}$. Os pixels $p$ e $q$ na imagem $\mathbf{b})$ possuem relação de adjacência 8-conectividade e seus conjuntos de adjacência são definidos como $\mathcal{A}_{8}(p)=\{(0,0),(0,2),(2,0),(2,2)\} \cup \mathcal{A}_{4}(p)$ e $\mathcal{A}_{8}(q)=$ $\{(4,4),(4,6),(6,4),(6,6)\} \cup \mathcal{A}_{4}(q)$.

Seja $f: \mathcal{D} \rightarrow \mathbb{K}$ uma imagem, $\mathcal{A}$ uma adjacência $\left(\mathcal{A}_{4}\right.$ ou $\left.\mathcal{A}_{8}\right)$ e $F \in \mathcal{F}(V, W)$ um mapeamento. Um grafo com peso nos vértices $G=(V, E, F)$ representa essa imagem se $V=\mathcal{D}, E=\mathcal{A}, W=\mathbb{K}$ e $F=f$. A Fig. 2.4 exibe um exemplo de imagem e suas representações como grafo. 


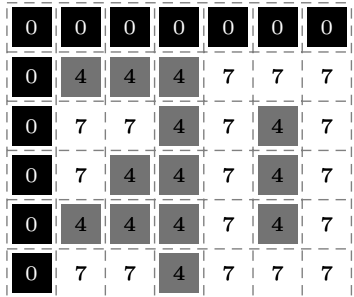

a) \begin{tabular}{l|l|l|l|l|l|l|l|l|l|l|l|l|l|l|l|l|l}
\hline 0 & 0 & 0 & 0 & 0 & 0 & 0 \\
\hline
\end{tabular}

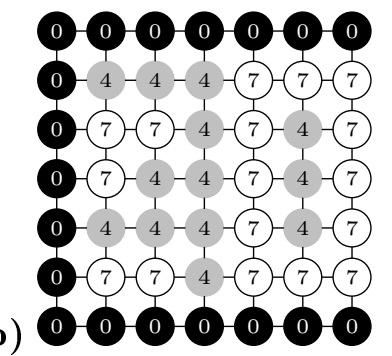

c)

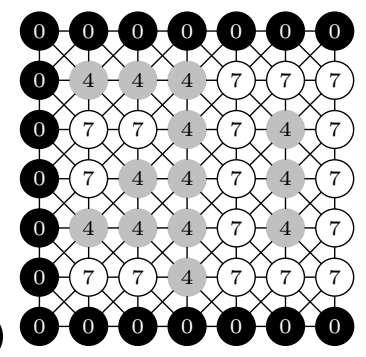

Fig. 2.4: Exemplo de uma imagem como grafo. a) imagem de exemplo; b) imagem a) como grafo com relação de adjacência 4-conectividade; c) imagem a) como grafo com relação de adjacência 8-conectividade. Os números nos nós representam seu peso (nivel de cinza) e a posiçâa de cada vértice representa sua coordenada na grade (dominio).

Na Fig. 2.4 os grafos apresentados representam a imagem a) e mais uma adjacência associada $\left(\mathcal{A}_{4}\right.$ e $\left.\mathcal{A}_{8}\right)$. Apesar das imagens dos grafos não explicitarem, cada vértice representa uma coordenada na grade $7 \times 7$ do $\mathbb{Z}^{2}$, ou seja, um pixel no domínio da imagem $\mathcal{D}$ e a mesma é representada no grafo pelo posicionamento do vértice. Portanto, o primeiro vértice da esquerda para a direita e de cima para baixo é o pixel $(0,0)$ e o último vértice é o pixel $(6,6)$.

Quando a imagem $f$ é binária, então o conjunto de vértices é representado pelo conjunto de pixels com valor 1 (ou seja, $V=\{p \in \mathcal{D}: f(p)=1\}$ ), podendo assim ser representada por um grafo não conexo. Neste caso o conjunto de componentes conexos da imagem é denotado por $\mathcal{C C}(V, \mathcal{A})$ e fornece informações que podem ser utilizadas para a análise da imagem, por exemplo, área e perímetro de cada componente, número de componentes e informações de forma de cada componente entre outras informações. A Fig. 2.5 exibe um exemplo de imagem binária representada por grafos de diferentes adjacências.

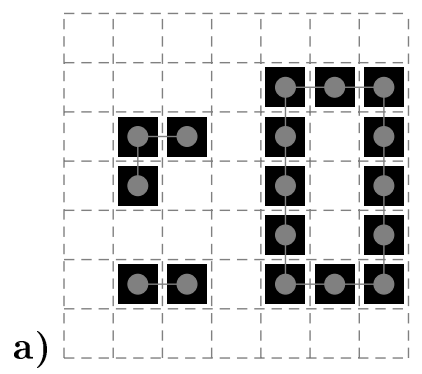

b)

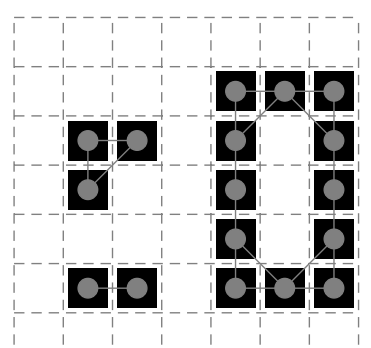

Fig. 2.5: Representações de imagens binárias como grafos. a) representação da imagem binária como um grafo utilizando a relação de adjacência de 4-conectividade; b) representação da imagem binária como um grafo com relação de adjacência de 8-conectividade. Os grafos estão inscritos nas imagens.

Os grafos apresentados na Fig. 2.5 têm 3 componentes conexos que podem ser utilizados para o cálculo de atributos e análise de imagem, por exemplo, com essa representação é possível extrair da imagem que ela possui 3 componentes conexos, com área 2, 3 e 12, existe um componente com buraco, entre outras informações. Embora nessa figura a relação de adjacência não tenha alterado os componentes conexos do grafo, existem casos que a adjacência pode muda-los. A Fig. 2.6 mostra um exemplo em que as informações dos componentes conexos de uma imagem representada como grafo são diferentes dependendo da relação de adjacência adotada. 


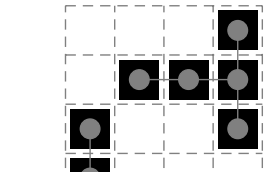

a)

b)

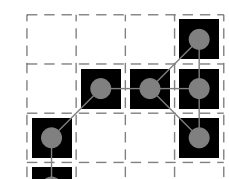

Fig. 2.6: Imagem binária representada por grafos com componentes conexos diferentes. Embora as imagens a) e b) sejam as mesmas, os grafos que as representam possuem componentes conexos diferentes. $O$ grafo da imagem a) tem 2 componentes conexos e o grafo da imagem b) é conexo.

Conforme exibido na Fig. 2.6 os componentes conexos dos grafos que representam a mesma imagem podem ser diferentes de acordo com a relação de adjacência, alterando assim informações da imagem, por exemplo, o grafo da imagem a) possui dois componentes conexos, um com área 2 e outro com área 5, por outro lado, mesmo utilizando a mesma imagem o grafo da imagem $\mathbf{b}$ ) possui apenas um componente conexo com 7 pixels de área. Portanto, a relação de adjacência é um parâmetro importante que pode alterar os resultados da análise da imagem quando a mesma é realizada em cima da representação de grafo.

\subsubsection{Limiarização e conjunto de níveis}

A limiarização é uma operação de processamento de imagens em níveis de cinza que seleciona um subconjunto de pixels para formar uma imagem binária. O método de limiarização consiste em escolher um valor de limiar no nível de cinza da imagem e selecionar todos os pixels que tenham intensidade maior (ou menor) que esse limiar, por exemplo, seja $f: \mathcal{D} \rightarrow \mathbb{K}$ uma imagem em níveis de cinza, e $\lambda$ um valor de limiar, a imagem binária resultante da operação de limiarização será $g=\{p \in \mathcal{D}: f(p) \geq \lambda\}$ ou $g=\{p \in \mathcal{D}: f(p) \leq \lambda\}$. Um exemplo da operação de limiarização é exibido na Fig. 2.7.

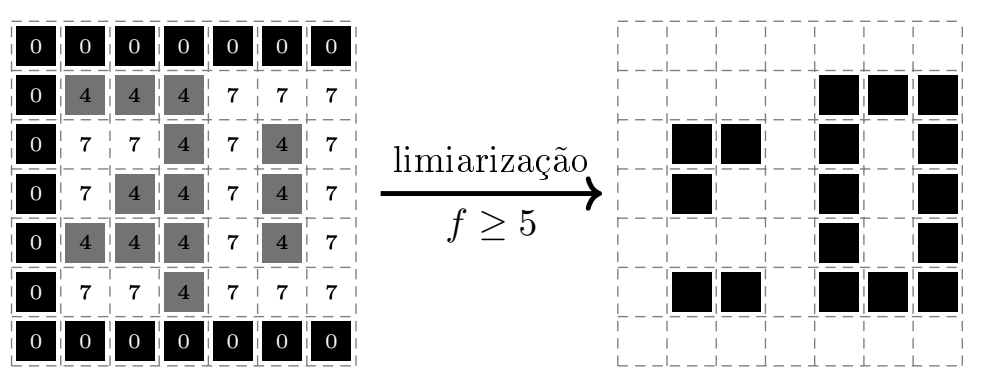

Fig. 2.7: Exemplo da operação de limiarização em uma imagem em níveis de cinza por um limiar de valor 5 .

A Fig. 2.7 exibe um exemplo de limiarização com um limiar $\lambda=5$ e para a operação de maior igual $(\geq)$. Apenas os pixels com intensidade maior ou igual a 5 são mantidos no conjunto que representa a imagem binária.

Apesar de existirem diversas técnicas derivadas da limiarização como: a limiarização adaptativa, e técnicas de escolher um valor de limiar como: o método de Otsu, a operação de limiarização desse trabalho é utilizada para definir o conjunto de níveis(level set). Dada uma imagem $f: \mathcal{D} \rightarrow \mathbb{K}$ o seu conjunto de nível é o resultado de uma operação de limiarização. Quando os pixels desejados são aqueles com intensidade abaixo de um limiar $\lambda$, então o conjunto resultante é chamado de conjunto de nivel inferior de valor $\lambda$ e o mesmo é denotado por $\mathcal{X}_{\downarrow}^{\lambda}(f)$ e quando os pixels desejados são aqueles com valor acima ou igual a um limiar $\lambda$, então o conjunto resultante é chamado de conjunto de nível superior de valor $\lambda$ e é denotado por $\mathcal{X}_{\lambda}^{\uparrow}(f)$. Por convenção, $\mathcal{X}_{\lambda}^{\uparrow}(f)=\{p \in \mathcal{D}: f(p) \geq \lambda\}$ e $\mathcal{X}_{\downarrow}^{\lambda}(f)=\{p \in \mathcal{D}: f(p)<\lambda\}$ a razão em manter os valores não-estritos e estritos para o conjunto de nível superior e inferior respectivamente, é referente a consistência nas operações que utilizam ambos os conjuntos, por exemplo, $\mathcal{D} \backslash \mathcal{X}_{\downarrow}^{\lambda}(f)=\mathcal{X}_{\lambda}^{\uparrow}(f)$.

O aninhamento de conjuntos de níveis é uma importante propriedade para a construção da árvore 
de componentes de uma imagem. Os conjuntos de níveis superiores de uma imagem $f: \mathcal{D} \rightarrow \mathbb{K}$ podem ser aninhados da seguinte forma:

$$
\mathcal{X}_{0}^{\uparrow}(f) \supseteq \mathcal{X}_{1}^{\uparrow}(f) \supseteq \ldots \supseteq \mathcal{X}_{K}^{\uparrow}(f),
$$

os conjuntos de níveis inferiores para a mesma imagem podem ser aninhados da seguinte forma:

$$
\mathcal{X}_{\downarrow}^{0}(f) \subseteq \mathcal{X}_{\downarrow}^{1}(f) \subseteq \ldots \subseteq \mathcal{X}_{\downarrow}^{K}(f) .
$$

A Fig. 2.8 apresenta um exemplo com os conjuntos de níveis superiores e inferiores e a propriedade de aninhamento.

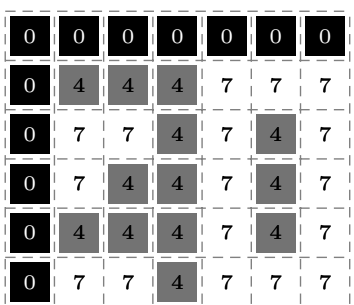

\section{\begin{tabular}{l|l|l|l|l|l|l|l|l|l}
0 & 0 & 0 & 0 & 0 & 0 & 0
\end{tabular}}

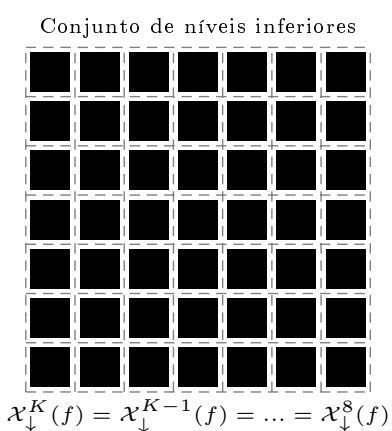

Imagem $f: \mathcal{D} \rightarrow \mathbb{K}$

Conjuntos de níveis superiores

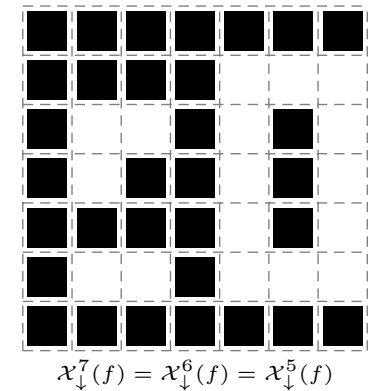

QDODOT QD口QDO D回回 QD口OD

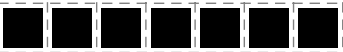
QD口QDO QDaDaD $\mathcal{X}_{0}^{\uparrow}(f)$
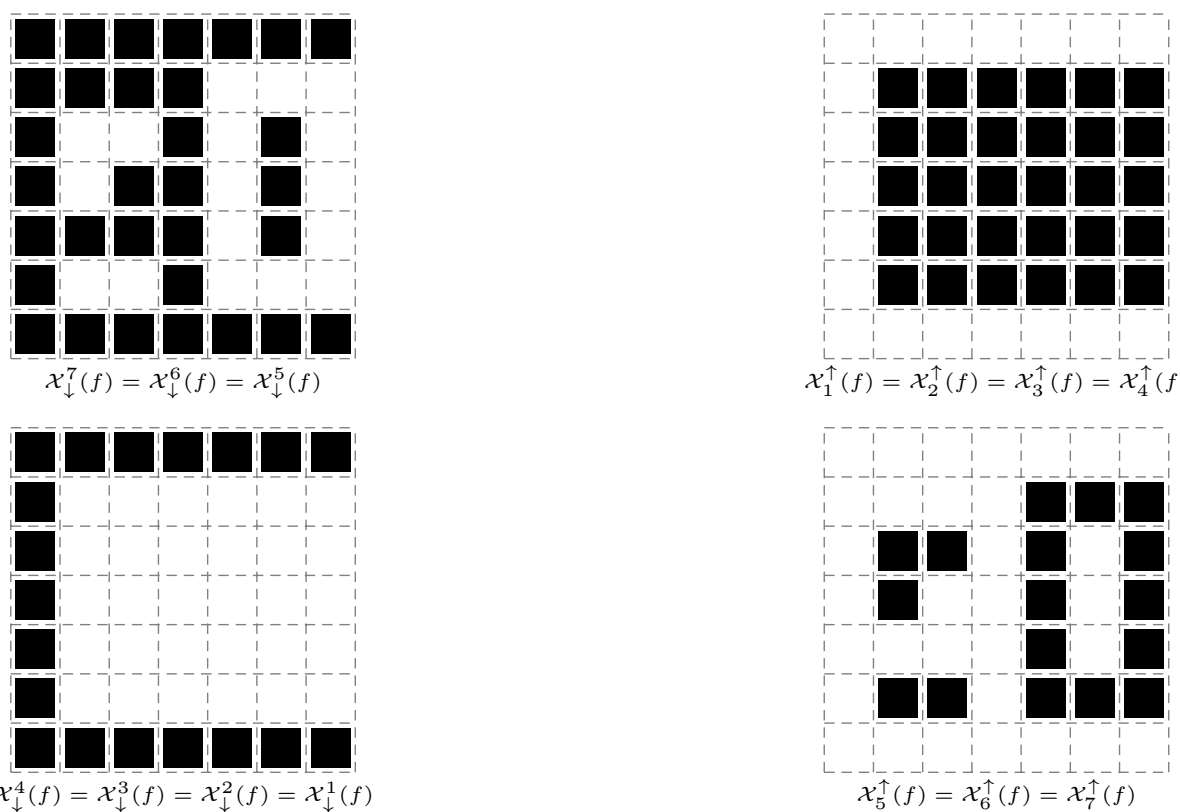

Fig. 2.8: Exemplo de conjuntos de níveis e da propriedade de aninhamento. As imagens da coluna esquerda apresentam os conjuntos de niveis inferiores e as da coluna direita apresentam os conjuntos de niveis superiores. As imagens estão aninhadas em relação à operação de inclusão de baixo para cima.

As imagens de exemplo da Fig. 2.8 exibem os valores de $\lambda$ que alteram os conjuntos de níveis. As imagens estão organizadas em duas colunas: a da esquerda representa os conjuntos de níveis inferiores e a da direita representa os conjuntos de níveis superiores. A propriedade de aninhamento 
está representada pela posição de cada imagem, tal que a imagem de baixo está inclusa na imagem a cima em seu respectivo conjunto de nível.

Dados os conjuntos de níveis de uma imagem $f: \mathcal{D} \rightarrow \mathbb{K}$ é possível reconstrui-la utilizando a propriedade de aninhamento. Considere $\alpha, \beta \in \mathbb{K}$, tal que $\alpha>\beta$, então pela propriedade de aninhamento é possível concluir que $\mathcal{X}_{\downarrow}^{\beta}(f) \subseteq \mathcal{X}_{\downarrow}^{\alpha}(f)$, portanto, selecionando um pixel $q \in \mathcal{D}$, e o conjunto de nível $\mathcal{X}_{\downarrow}^{f(q)}(f)=\{p \in \mathcal{D}: f(p)<f(q)\}$ é concluido que $q \notin \mathcal{X}_{\downarrow}^{f(q)}(f)$ e graças a propriedade de aninhamento segue que $q \notin \mathcal{X}_{\downarrow}^{f(q)-1}(f) \supseteq \mathcal{X}_{\downarrow}^{f(q)-2}(f) \supseteq \ldots \supseteq \mathcal{X}_{\downarrow}^{0}(f)$. Então dados os conjuntos de níveis inferiores de uma imagem é possível recuperar o nível de cinza de um pixel $q$ utilizando a seguinte relação $f(q)=\min \left\{\lambda \in \mathbb{K}: q \in \mathcal{X}_{\downarrow}^{\lambda}(f)\right\}-1$. Analogamente, é possível recuperar a intensidade de um pixel $q$ utilizando somente os conjuntos de níveis superiores através da seguinte relação: $f(q)=\max \left\{\lambda \in \mathbb{K}: q \in \mathcal{X}_{\lambda}^{\uparrow}(f)\right\}$. Um exemplo de como são recuperadas as intensidades de alguns pixels selecionados é exibido na Fig. 2.9.

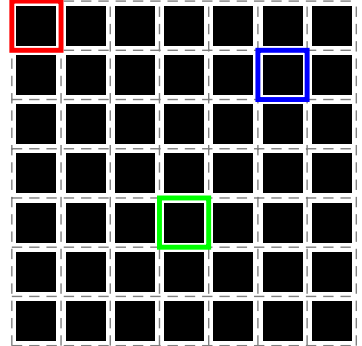

$\mathcal{X}_{0}^{\uparrow}(f)$

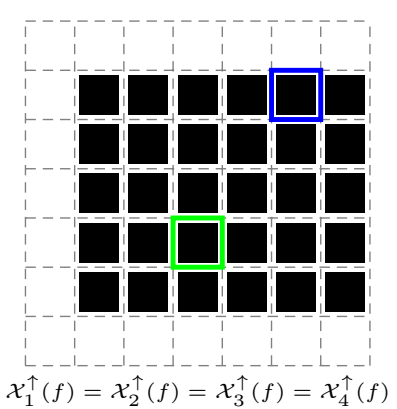

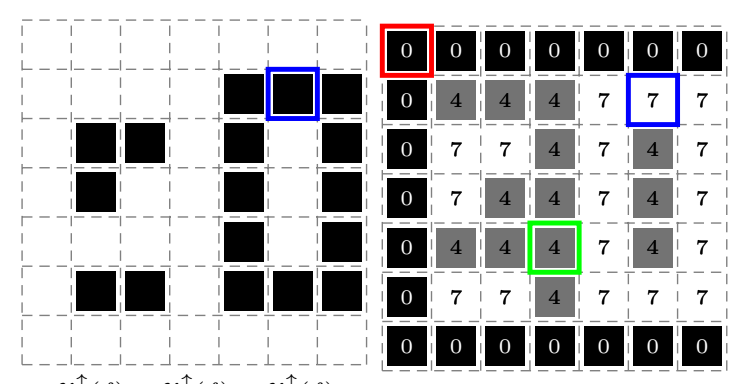

$\mathcal{X}_{5}^{\uparrow}(f)=\mathcal{X}_{6}^{\uparrow}(f)=\mathcal{X}_{7}^{\uparrow}(f)$ $f: \mathcal{D} \rightarrow \mathbb{K}$

Fig. 2.9: Exemplo de reconstrução de uma imagem através de seus conjuntos de níveis superiores. Na primeira imagem são marcados os pixels de exemplo no conjunto $\mathcal{X}_{0}^{\uparrow}(f)$. A partir do conjunto $\mathcal{X}_{1}^{\uparrow}(f)$ o pixel vermelho está fora do conjunto, a partir do conjunto $\mathcal{X}_{5}^{\uparrow}(f)$ o pixel verde. No final a imagem em níveis de cinza é reconstruída.

Na Fig. 2.9 são marcados 3 pixels que podem ser nomeados como $p$ marcado em vermelho, $q$ marcado em verde e $r$ marcado em azul. O pixel $p$ só pertence a $\mathcal{X}_{0}^{\uparrow}(f) \operatorname{logo} f(p)=0$, o pixel $q$ pertence aos conjuntos $\mathcal{X}_{0}^{\uparrow}(f) \subseteq \mathcal{X}_{1}^{\uparrow}(f)=\mathcal{X}_{2}^{\uparrow}(f)=\mathcal{X}_{3}^{\uparrow}(f)=\mathcal{X}_{4}^{\uparrow}(f)$, mas não pertence ao $\mathcal{X}_{5}^{\uparrow}(f)$, como seu conjunto de nível superior máximo é 4 , então $f(q)=4$. Similarmente, o pixel $r$ tem $\mathcal{X}_{7}^{\uparrow}(f)$ como o conjunto de nível superior máximo e consequentemente, $f(r)=7$. Mesmo apresentando apenas 3 exemplos, o mesmo ocorre para os outros pixels dependendo apenas de seu conjunto de nível superior com o valor máximo para encontrar a sua intensidade.

\section{2 Árvore de componentes}

\subsubsection{Conceitos}

Uma árvore de componentes é uma representação completa de uma imagem em níveis de cinza que organiza os componentes conexos dos conjuntos de níveis em uma árvore (grafo conexo acíclico) de acordo com o aninhamento desses conjuntos.

Seja $f: \mathcal{D} \rightarrow \mathbb{K}$ uma imagem, a árvore de componentes de $f$ é construída em cima de todos os componentes conexos de todos os conjuntos de níveis (superiores ou inferiores) dessa imagem. Defina-se o conjunto $\mathcal{L}(f)=\left\{C \in \mathcal{C C}\left(\mathcal{X}_{\lambda}^{\downarrow}(f), \mathcal{A}\right): \lambda \in \mathbb{K}\right\}$ como o conjunto de todos os componentes conexos dos conjuntos de níveis inferiores e o conjunto $\mathcal{U}(f)=\left\{C \in \mathcal{C C}\left(\mathcal{X}_{\uparrow}^{\lambda}(f), \mathcal{A}\right): \lambda \in \mathbb{K}\right\}$ como o conjunto de todos os componentes conexos dos conjuntos de níveis superiores. Dadas as definições de $\mathcal{U}(f)$ e $\mathcal{L}(f)$, observa-se que os componentes conexos dos conjuntos de níveis, ou estão aninhados, ou estão disjuntos, ou seja, para $\alpha, \beta \in \mathbb{K}$, tal que $\alpha \geq \beta, A \in \mathcal{C C}\left(\mathcal{X}_{\uparrow}^{\alpha}(f), \mathcal{A}\right)$ e $B \in \mathcal{C C}\left(\mathcal{X}_{\uparrow}^{\beta}(f), \mathcal{A}\right)$, então ou $A \cap B=\emptyset$ ou $B \supseteq A$, isso pode ser verificado pelo fato que se existisse uma intersecção não vazia entre $A$ e $B$, então pela propriedade de aninhamento (Sec. 2.1.4) $A \subseteq B$. Similarmente, para qualquer $A \in \mathcal{C C}\left(\mathcal{X}_{\alpha}^{\downarrow}(f), \mathcal{A}\right)$ e $B \in \mathcal{C C}\left(\mathcal{X}_{\beta}^{\downarrow}(f), \mathcal{A}\right)$, então ou $A \cap B=\emptyset$, ou $B \subseteq A$. 
Cada componente $C \in \mathcal{U}(f)$ pode ser associado a uma intensidade de $f$ que gerou o componente, por exemplo, seja $C \in \mathcal{X}_{\uparrow}^{\lambda}(f)$, então a intensidade associada a esse componente é $\lambda$. Caso o valor de limiar não seja conhecido, esse valor pode ser obtido pela seguinte operação: $\lambda=\min \{f(p): p \in C\}$. Similarmente se $C \in \mathcal{X}_{\lambda}^{\downarrow}(f)$ (consequentemente $C \in \mathcal{L}(f)$ ), então sua intensidade associada é $\lambda=\max \{f(p): p \in C\}+1$.

A árvore de componentes $T(f)$ ou somente $T$ quando $f$ estiver implícita, é formada por nós que consistem de duplas $(C, \lambda)$ onde $C \in \mathcal{U}(f)$ ou $\mathcal{L}(f)$ e $\lambda$ é a intensidade associada ao componente $C$, mais uma relação de parentesco $P$, onde dados dois nós $(A, \alpha)$ e $(B, \beta)$ (para $\alpha, \beta \in \mathbb{K}$ ), $(A, \alpha) P(B, \beta)$ se e somente se, $B \subset A$ e não existe um nó $(D, \gamma)$ (para $\gamma \in \mathbb{K}$ ), tal que $B \subset D \subset A$. Dada que a relação $(A, \alpha) P(B, \beta)$ existe, então $(A, \alpha)$ é chamado de nó pai de $(B, \beta)$ e $(B, \beta)$ é chamado de nó filho de $(A, \alpha)$. Os valores $\lambda, \alpha, \beta$ e $\gamma$ são chamados de altitude de seus respectivos nós. Um nó da árvore também é denotado apenas por seu componente conexo quando sua altitude não é utilizada no contexto, por exemplo, o nó $(C, \lambda)$ é denotado apenas por $C$ quando $\lambda$ não é uma informação necessária no contexto. Toda árvore de componentes possui um único nó que não possui um nó pai, esse nó é chamado de nó raiz. Similarmente, toda árvore de componentes possui nós que não tem nós filhos, esses nós são chamados de nós folhas. Dados dois nós $A_{0}$ e $A_{n}$, o nó $A_{n}$ é chamado de nó descendente de $A_{0}$, se existe um caminho $\pi=\left(A_{0}, A_{1}, A_{2}, \ldots, A_{n}\right)$, tal que $A_{i} P A_{i+1}, \forall i \in\{0,1, \ldots, n-1\}$, neste caso o nó $A_{0}$ é chamado de nó ancestral de $A_{n}$. A altura de um nó na árvore é a distância do nó até o seu nó descendente mais distante. Apresentados os conceitos sobre árvore de componentes, são formuladas as seguintes propriedades:

Propriedade 1. Todo nó folha de uma árvore de componentes construída a partir dos conjuntos de níveis superiores é um máximo local, ou seja, um nó folha representa uma região de intensidade constante, tal que nenhum pixel dessa região tem intensidade menor que qualquer um de seus pixels vizinhos.

Propriedade 2. Todo nó folha de uma árvore de componentes construída a partir dos conjuntos de níveis inferiores é um minimo local, ou seja, um nó folha representa uma região de intensidade constante, tal que nenhum pixel dessa região tem intensidade maior que qualquer um de seus pixels vizinhos.

Propriedade 3. Todo nó folha de uma árvore tem altura 0.

A Fig. 2.10 exibe exemplos de árvores de componentes utilizando conjuntos de níveis superiores e inferiores.

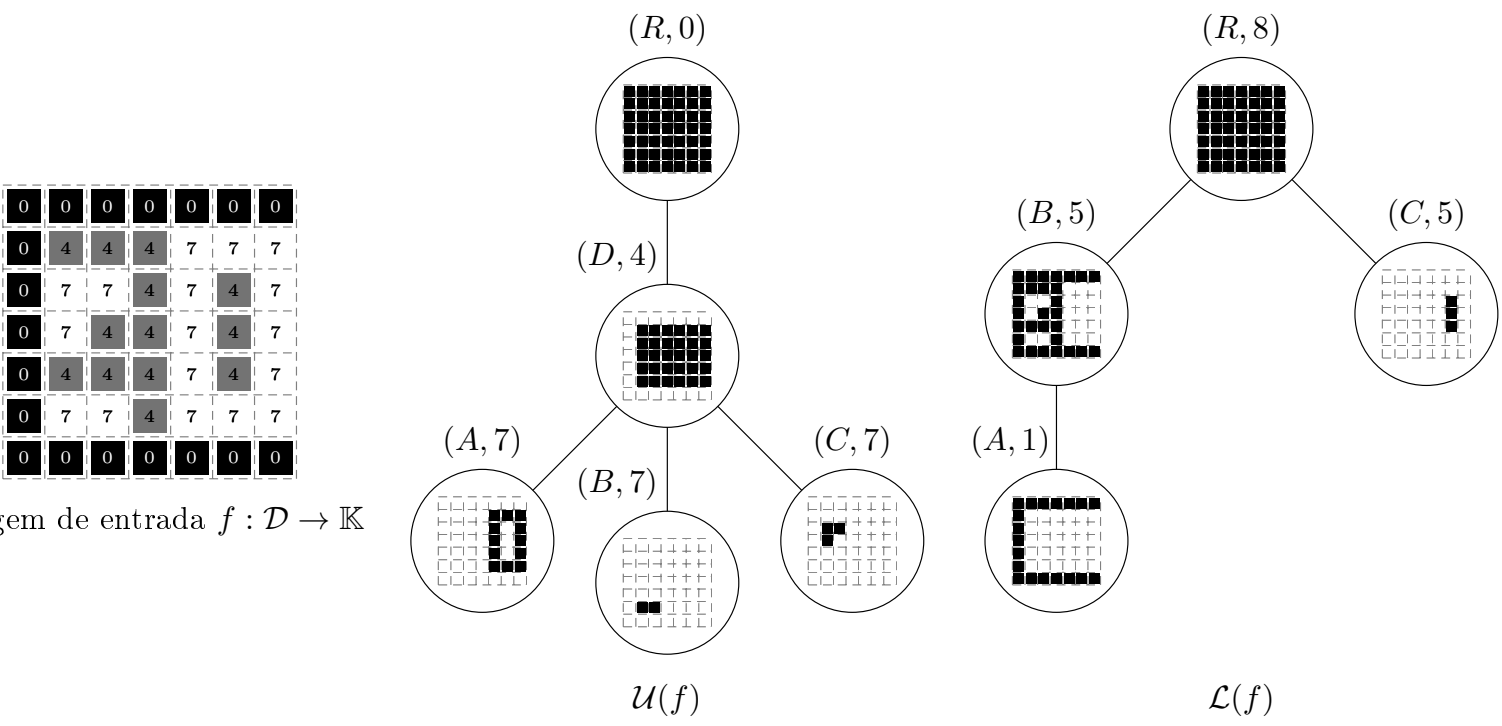

Fig. 2.10: Exemplos de árvores de componentes com os conjuntos de niveis superiores $\mathcal{U}(f)$ e inferiores $\mathcal{L}(f)$. 
A árvore de componentes utilizando o conjunto $\mathcal{U}(f)$ da Fig. 2.10 possui os nós $(R, 0),(D, 4)$, $(C, 7),(B, 7),(A, 7)$ e a relação de parentesco indica que $A \subset D, B \subset D, C \subset D$ e $D \subset R$. Apesar dos nós $A, B$ e $C$ não serem nós filhos de $R$, a relação de parentesco entre os mesmos com $D$ e de $D$ com $R$ indicam que $A \subset R, B \subset R, C \subset R$. Nesse exemplo, os nós $A, B$ e $C$ tem altura 0 , o nó $D$ tem altura 1 e $R$ tem altura 2. Similarmente a árvore construída em cima do conjunto $\mathcal{L}(f)$ possui os nós $(R, 8),(C, 5),(B, 5),(A, 1)$ e a relação de parentesco indica que $A \subset B, B \subset R$ e $C \subset R$, e consequentemente $A \subset R$. Nessa árvore os nós $A, C$ tem altura 0 , o nó $B$ tem altura 1 e o nó $R$ possui altura 2 .

Embora a árvore de componentes seja utilizada em diversas aplicações para análise e processamento de imagens, na prática, o armazenamento de todos os conjuntos de níveis geralmente é uma operação custosa em relação a memória de computadores. A implementação comum da árvore de componentes utiliza uma representação compacta e não redundante chamada de max-tree quando a árvore de componentes é construída em cima do conjunto $\mathcal{U}(f)$, ou de min-tree quando a árvore é construída em cima do conjunto $\mathcal{L}(f)$. A max-tree ou a min-tree é denotada por $\mathcal{T}(T)$ ou somente $\mathcal{T}$, quando $T$ está implícita e a mesma é formada por nós compactos e não redundantes que estão associados aos nós da árvore de componentes que está sendo representada. A relação de parentesco dos nós de uma árvore compacta e não redundante são as mesmas de seus respectivos nós associados na árvore de componentes, por exemplo, seja $T$ uma árvore de componentes, $V_{T}$ os nós de $T, \mathcal{T}$ a representação compacta e não redundante de $T$ e seja $(C, \lambda) \in V_{T}$ um nó de $T$, então existe um nó $(\hat{C}, \lambda) \in V_{\mathcal{T}}$, tal que $\hat{C}=\{p \in C: f(p)=\lambda\}$ se $\mathcal{T}$ é a max-tree e $\hat{C}=\{p \in C: f(p)=\lambda-1\}$ se $\mathcal{T}$ é a min-tree, neste caso, $\hat{C}$ está associado a $C$ e vice-versa. Para as mesmas árvores $T$ e $\mathcal{T}$, para cada par de nós $A, B \in V_{T}$ e seus respectivos nós associados $\hat{A}, \hat{B} \in V_{\mathcal{T}}$, o nó $\hat{A}$ é pai do nó $\hat{B}$, se e somente se, o nó $A$ é pai do nó $B$, ou seja, $B \subset A$.

É fácil verificar que o espaço gasto com a utilização da max-tree ou da min-tree é $\Theta(|\mathcal{D}|)$, porque cada pixel é armazenado em apenas um nó da árvore. O menor componente conexo de um pixel $p \in \mathcal{D}$ é o componente conexo (ou nó de uma árvore de componentes) que o contém e tem o menor número de pixels. O menor componente conexo de um pixel $p$ possui duas propriedades: $(i)$ os nós descendentes do nó que representa o menor componente não contém $p$ e $(i i)$ o nó da árvore compacta e não redundante que armazena o pixel p é o nó associado ao nó que representa o menor componente de $p$ na árvore de componentes. Seja $A$ um nó da árvore de componentes $T, \hat{A}$ um nó da árvore compacta e não resultante $\mathcal{T}$ de $T$ associado a $A$ e $p \in \mathcal{D}$ um pixel da imagem que gerou $T$, portanto, se $p \in \hat{A}$, então $A$ é o menor componente conexo de $p$, e o mesmo é denotado por $\mathcal{S C}(\mathcal{T}, p)=A$. A Fig. 2.11 ilustra um exemplo de max-tree e min-tree. 


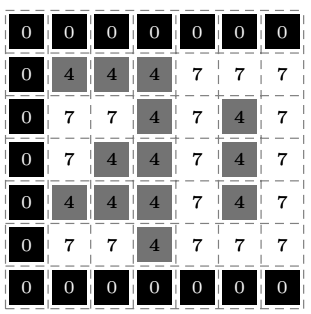

Imagem de entrada $f: \mathcal{D} \rightarrow \mathbb{K}$

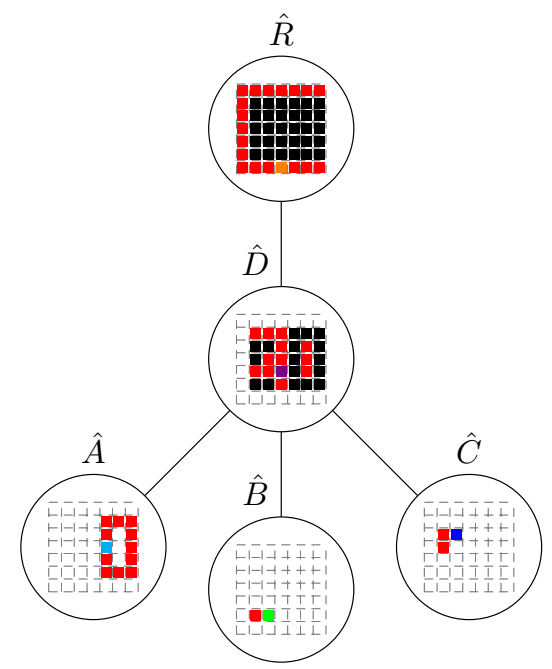

max-tree

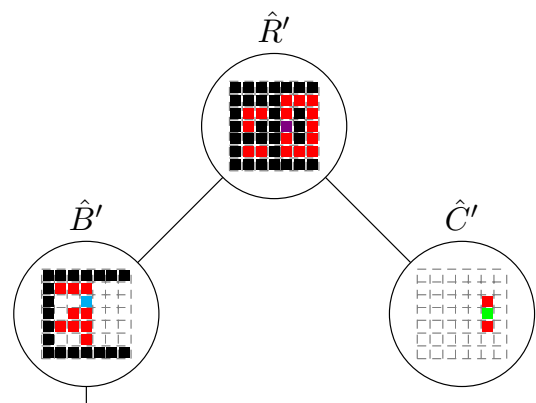

min-tree

Fig. 2.11: Exemplo de max-tree e min-tree de uma dada imagem de entrada. Os pixels pretos representam pixels do nó na árvore de componentes que não são armazenados em seu respectivo nó associado, os pixels brancos são pixels do domínio que não pertencem ao nó e os outros pixels são os pixels armazenados nos nós da max-tree ou min-tree.

Na Fig. 2.11, os pixels brancos nos nós das árvores representam os pixels do domínio da imagem que não pertencem ao nó, os pixels pretos pertencem ao nó da respectiva árvore de componentes e não são armazenados no nó associado na árvore compacta e não redundante, os pixels nem preto e nem branco são armazenados na max-tree ou min-tree. Seja $\mathcal{T}$ a max-tree na Fig. 2.11 e $p, q, r, s, t$ os pixels de cor laranja, roxo, azul-claro, verde e azul-escuro na max-tree respectivamente, então, $\mathcal{S C}(\mathcal{T}, p)=R, \mathcal{S C}(\mathcal{T}, q)=D, \mathcal{S C}(\mathcal{T}, r)=A, \mathcal{S C}(\mathcal{T}, s)=B, \mathcal{S C}(\mathcal{T}, t)=C$, onde os nós $A, B$, $C, D, R$ são os nós associados dos nós $\hat{A}, \hat{B}, \hat{C}, \hat{D}, \hat{R}$ respectivamente na árvore de componentes representada por $\mathcal{T}$. Similarmente, na min-tree $\mathcal{T}^{\prime}$ da Fig. 2.11, os pixels $p^{\prime}, q^{\prime}, r^{\prime}, s^{\prime}$ são os pixels de cor roxo, azul-claro, verde e azul-escuro na min-tree respectivamente, portanto, $\mathcal{S C}\left(\mathcal{T}^{\prime}, p^{\prime}\right)=R^{\prime}$, $\mathcal{S C}\left(\mathcal{T}^{\prime}, q^{\prime}\right)=B^{\prime}, \mathcal{S C}\left(\mathcal{T}^{\prime}, r^{\prime}\right)=C^{\prime}, \mathcal{S C}\left(\mathcal{T}^{\prime}, s^{\prime}\right)=A^{\prime}$, onde os nós $A^{\prime}, B^{\prime}, C^{\prime}, R^{\prime}$ são os nós associados aos nós $\hat{A}^{\prime}, \hat{B}^{\prime}, \hat{C}^{\prime}, \hat{R}^{\prime}$ respectivamente na árvore de componentes representada por $\mathcal{T}^{\prime}$.

\subsubsection{Computação incremental de atributos}

Um atributo é uma função que mapeia um conjunto de pixels a um valor numérico que descreva alguma característica desse conjunto, ou seja, um atributo é uma função $\kappa: \mathcal{P}(\mathcal{D}) \rightarrow \mathbb{R}$ que representa informações de um conjunto de pixels como: área, altura, largura, perímetro, circularidade, entre outros. Esses atributos são geralmente utilizados para a descrição de elementos (geralmente componentes conexos) em imagens e criação filtros conexos. Em particular o uso de atributos para descrever nós da árvore de componentes é um recurso interessante para imagens em níveis de cinza com aplicações em reconhecimento de padrões, filtragem entre outros.

Quando os atributos são calculados em todos os nós da árvore de componentes, o processamento pode ser custoso se o cálculo utilizar todos os pixels de todos os nós da árvore, por outro lado, alguns atributos podem ser calculados incrementalmente nos nós de uma max-tree ou min-tree. Na computação incremental, o atributo de um nó da árvore de componentes é calculado utilizando: $(i)$ o conjunto de pixels armazenado em seu nó associado (max-tree ou min-tree) e (ii) os valores de atributo de seus nós filhos. Em resumo, o cálculo incremental de atributos é computado realizando um percurso de pós-ordem na max-tree (min-tree) começando o cálculo pelas folhas e utilizando uma operação de fusão de dados entre um nó pai e seus nós filhos. O Alg. 1 exibe um algoritmo geral para o cálculo incremental de atributos. 

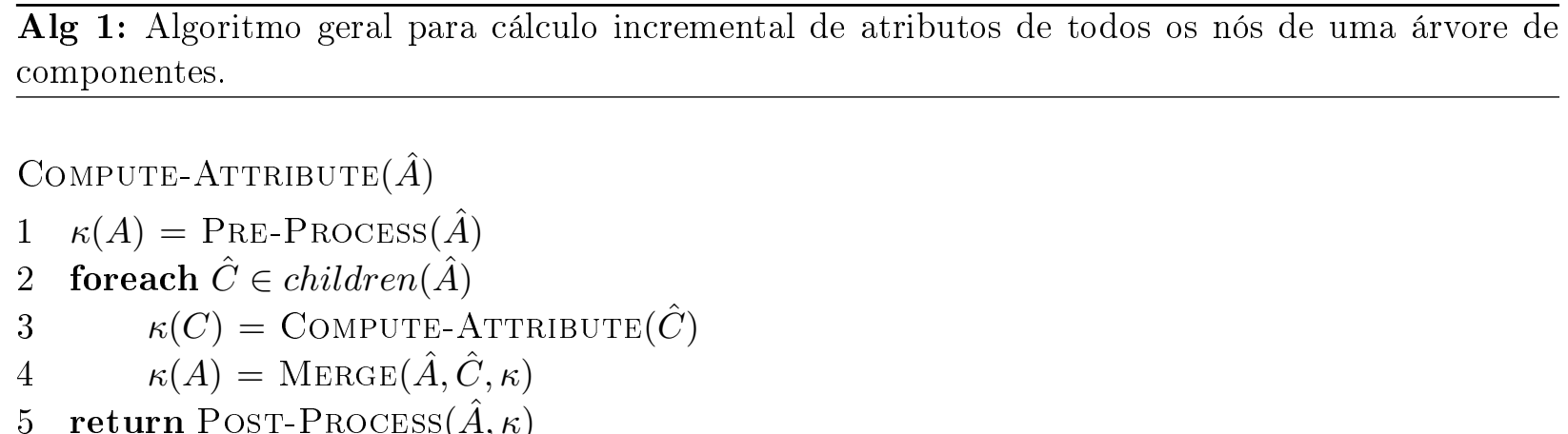

Utilizando uma abordagem bottom-up, o Alg. 1 recebe uma subárvore da max-tree (min-tree) de raiz $\hat{A}$ e calcula o atributo de todos os seus nós (atributo referente aos seus nós associados na árvore de componentes). O algoritmo inicia calculando o valor de atributo utilizando apenas os pixels do nó $\hat{A}$ da max-tree por meio da função PRE-PROCESS (veja linha 1), então o algoritmo percorre a árvore executando um laço nos nós filhos do nó $\hat{A}$ (veja linha 2, onde children $(\hat{A})$ é conjunto de nós filhos do nó $\hat{A}$ ), em seguida, cada iteração do laço calcula o atributo para um nó filho do nó $\hat{A}$ com a chamada a Compute-AtTribute (veja linha 3 ) que percorre a subárvore do nó até chegar a um nó folha e calcula o atributo dos nós dessa subárvore recursivamente. Com o atributo do nó filho calculado, o algoritmo executa uma operação de fusão com a chamada de Merge (veja linha 4), onde o atributo do nó $\hat{A}$ e o de seu nó filho $\hat{C}$ são combinados e o resultado dessa combinação é armazenado em $\kappa(\hat{A})$. A linha 5 do algoritmo executa um pós-processamento (chamando Post-PROCEss) necessário para alguns atributos que precisam ser ajustados após a combinação dos atributos de todos os nós filhos com o atributo de seu nó pai. Ao final, o algoritmo devolve o valor de atributo para cada nó da subárvore.

A área de um componente conexo é um atributo incremental, pois, existe um algoritmo que a computa incrementalmente. Utilizando a função ComPUte-ATTRIBUTE apresentada no Alg. 1, o Alg. 2 apresenta a implementação da função PRE-Process do algoritmo que calcula a área incrementalmente para todos os nós de uma árvore de componentes.

Alg 2: Função PRE-PRocess do algoritmo incremental para o cálculo da área na árvore de componentes.

$\operatorname{Pre}-\operatorname{Process}(\hat{A})$

1 return $|\hat{A}|$

No Alg. 2, a função Pre-Process simplesmente devolve o tamanho do conjunto de pixels armazenado pelo nó da árvore de componentes compacta e não redundante. Para qualquer nó folha de uma árvore compacta e não redundante esse é o valor da área, porém, para nós não folha é necessário o desenvolvimento de uma função de combinação, porque esses nós não armazenam o conjunto de pixels inteiro que representa o nó associado na árvore de componentes. Observe que se o algoritmo tivesse recebendo um nó da árvore de componentes a função PRE-PROCEss já resultaria na área do nó, porém, a árvore de componentes tem custo de armazenamento muito alto (veja Seção 2.2.1). A função que combina as áreas calculadas nos nós filhos com o valor intermediário da área calculada no respectivo nó pai é apresentada no Alg. 3. 
Alg 3: Função MERGE do algoritmo incremental para o cálculo da área na árvore de componentes.

$\operatorname{MeRGE}(\hat{A}, \hat{C}, \kappa)$

1 return $\kappa(A)+\kappa(C)$

A combinação da área de um nó pai com a área de um de seus nós filhos é mostrada no Alg. 3. A operação de Merge neste caso, é uma soma da área do nó pai que corresponde a quantidade de pixels de seu nó mais a área de todos os nós filhos percorridos até o momento, mais a área do nó filho que está sendo analisado na iteração. A área final do nó $\hat{A}$ será a somatória da área de todos os seus filhos mais a quantidade de pixels que $\hat{A}$ armazena. Para esse algoritmo, a função de Post-Process não é necessária, porque nenhuma operação após a combinação da área do nó pai com todas as áreas de seus nós filhos deve ser realizada. Portanto, a função Post-Process simplesmente retorna o valor já calculado da área, conforme mostrada no Alg. 4.

Alg 4: Função Post-Process do algoritmo incremental para o cálculo da área na árvore de componentes.

$\operatorname{Post-Process}(\hat{A}, \kappa)$

1 return $\kappa(A)$

Utilizando as funções definidas nos Algs. 2, 3, 4 como as funções Pre-Process, Merge e Post-Process respectivamente, o resultado do Alg. 1 será a área (quantidade de pixels) de cada nó da subárvore que tem o nó $A$ como raiz. Dado um nó $A$ da árvore de componentes o funcionamento do algoritmo pode ser resumido na seguinte equação:

$$
\kappa(\hat{A})=|\hat{A}|+\sum_{\hat{C} \in \operatorname{children}(\hat{A})} \kappa(\hat{C})=|A| .
$$

A demonstração que essa equação resulta na área do nó é exibida abaixo:

Prop 2.1. Seja $f: \mathcal{D} \rightarrow \mathbb{K}$ uma imagem, $T$ a árvore de componentes construída a partir de $f, \mathcal{T}$ a árvore compacta e não redundante de $T, V_{T}$ o conjunto de nós de $T, V_{\mathcal{T}}$ o conjunto de nós de $\mathcal{T}$, $A \in V_{T}$ um nó de $T$ e $\hat{A} \in V_{\mathcal{T}}$ o seu respectivo nó associado na árvore de componentes compacta $e$ não redundante. Então a área (quantidade de pixels) do nó Â é dada por:

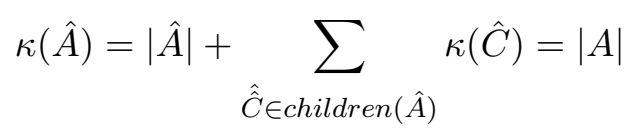

Demonstração. A prova para a proposição é dada por indução na altura $h$ do nó $A$.

- Caso Base: $h=0$.

Quando o nó $A$ tem altura 0, então o nó é uma folha e portanto a proposição é trivialmente verdade porque $\hat{A}=A$ e $\sum_{\hat{C} \in \operatorname{children}(A)} \kappa(\hat{C})=0$ (porque $\operatorname{children}(\hat{A})=\emptyset$ ).

- Hipótese de indução: $h<n$.

Suponha que a proposição é verdadeira quando o nó $A$ tem altura menor que $n$ (para $n \in \mathbb{N}$ ).

- Prova: $h=n$

Seja $A$ um nó de altura $n>0$, então todos os seus nós filhos têm no máxima altura $n-1$, portanto por hipótese de indução é possível concluir que $\forall C \in \operatorname{children}(\hat{A}), \kappa(\hat{C})=|C|$. 
Seja $\mathcal{C}=\cup_{C \in \operatorname{children}(A)} C$, por definição $\forall p \in A$ se $\mathcal{S C}(\mathcal{T}, p) \neq A$, então $p \notin \hat{A}$ e $p$ está em um descendente de $A$, ou seja, $p \in \mathcal{C}$, senão $(\mathcal{S C}(\mathcal{T}, p)=A), p \in \hat{A}$, logo $\hat{A}=A \backslash \mathcal{C}$, pela propriedade do aninhamento dos conjuntos de níveis, quaisquer dois nós em children $(A)$ são disjuntos entre si, então $|\mathcal{C}|=\sum_{\hat{C} \in \operatorname{children}(\hat{A})} \kappa(\hat{C})$. Portanto se conclui que $|A|=|\hat{A}|+|\mathcal{C}|=$ $|\hat{A}|+\sum_{\hat{C} \in \operatorname{children}(\hat{A})} \kappa(\hat{C})$.

Como o Alg. 1 é recursivo, é possível calcular a área de todos os nós de uma árvore de componentes, passando a raiz da árvore como argumento. Um exemplo da execução do algoritmo incremental para o cálculo da área na árvore de componentes é apresentado na Fig. 2.12.

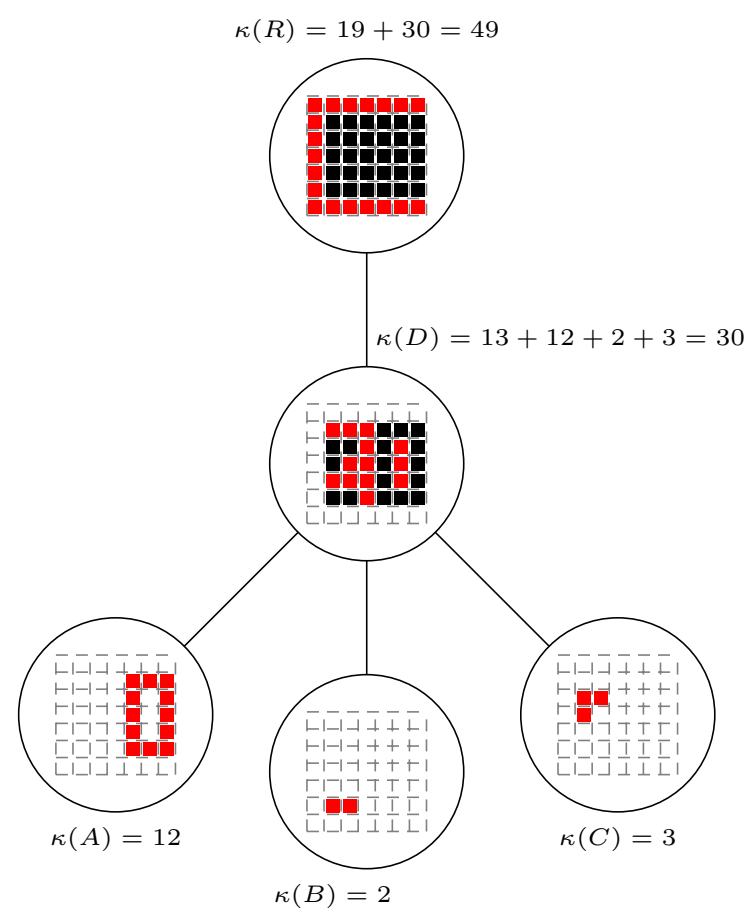

max-tree

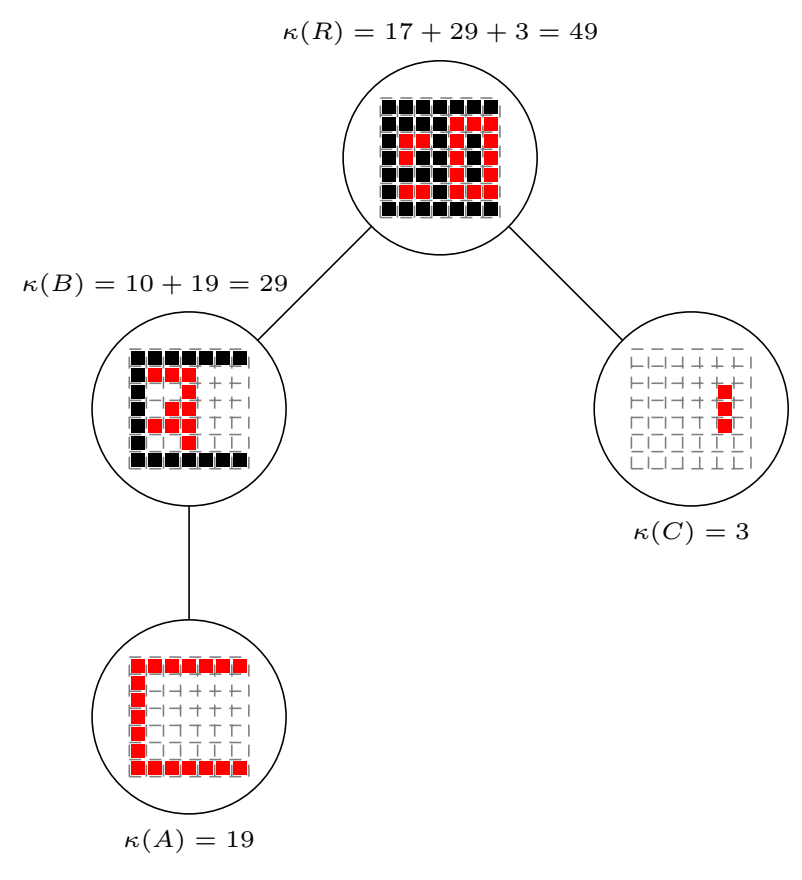

min-tree

Fig. 2.12: Exemplo do algoritmo que calcula incrementalmente a área de todos os nós de uma árvore de componentes. Nos nós folhas a área é exibida abaixo dos nós, em nós não folha a área é exibida acima do nó, onde o primeiro valor da esquerda para a direita indica a quantidade de pixels na árvore compacta e não redundante (fim do Alg. 2) e os seguintes valores indicam a área de cada nó filho (cada iteração do Alg. 3). Os pixels em vermelho indicam os pixels armazenados na max-tree ou min-tree, os pixels pretos indicam os pixels do nó na árvore de componentes que não são armazenados em seus nós associados e os pixels brancos indicam os pixels do domínio da imagem que não pertencem ao nó.

Para a max-tree representada na Fig. 2.12, o algoritmo apresentado começa calculando um valor intermediário para as áreas dos nós $R$ e $D$ respectivamente, como $\kappa(R)=19$ e $\kappa(D)=13$, então são calculadas as áreas dos nós filhos de $D$, começando pelo nó $A$ que não possui nós filhos e consequentemente tem sua área calculada por uma chamada a PRE-Process resultando em $\kappa(A)=12$. As áreas do nó $A$ e do nó $D$ são acumuladas na operação de MERGE resultando em $\kappa(D)=13+12=25$, em seguida o algoritmo calcula a área do nó $B$ diretamente resultando em $\kappa(B)=2$ e depois a função Merge acumula os valores em $\kappa(D)=25+2=27$. Antes de finalizar o laço nos nós filhos de $D$, a área do nó $C$ é calculada em $\kappa(C)=3$ e acumulada em $\kappa(D)=27+3=30$. Então o laço de chamada recursivas para os nós filhos de $D$ é finalizado e o algoritmo volta para a finalizar o cálculo da área do nó $R$, através de uma chamada a MERGE 
que realiza a soma $\kappa(R)=19+30=49$, obtendo assim a área final para cada nó na max-tree. A execução do algoritmo na min-tree é análogo.

O algoritmo gasta tempo linear no número de nós da árvore $\left(\Theta\left(\left|V_{\mathcal{T}}\right|\right)\right)$ se for considerado que a operação de obter o tamanho de um conjunto é constante, senão o algoritmo gasta tempo linear no número de pixels $(\Theta(|\mathcal{D}|))$ se for considerado que a operação de obter o tamanho do conjunto de pixels gasta tempo constante para cada pixel do conjunto.

\subsection{Cálculo de atributos utilizando bit-quads}

\subsubsection{Contagem de bit-quads em imagens binárias}

O algoritmo para o cálculo de atributos de imagens binárias utilizando bit-quads é baseado em um conjunto de definições no espaço contínuo que podem ser interpretadas no espaço discreto (normalmente utilizado para a representação de imagens). Gray descreve o algoritmo realizando as seguintes definições para imagens $2 D$ [Gra71]:

- Borda: É uma curva simples fechada.

- Conjunto de bordas: É um conjunto finito de bordas que não se tocam ou se intersectam.

- Região Binária: É uma área finita conexa no plano, sem bordas que a cruza e tem uma atribuição de valor 0 ou 1.

- Exterior: É uma região binária que fica fora do conjunto de bordas definidas no plano.

- Paridade: É definido pela quantidade de linhas que são cruzadas por qualquer caminho de um ponto ao exterior não tangente a nenhuma borda (a quantidade pode ser par ou ímpar).

- Imagem binária: É um plano que possui um conjunto de bordas onde todo ponto de paridade par tem valor 0 e paridade ímpar tem valor 1.

- Borda de objeto: É uma borda onde os pontos interiores têm valor 1.

- Borda de buraco: É uma borda onde os pontos interiores têm valor 0 .

- Buraco: É a região interna da borda de buraco.

- Objeto: É a região interna da borda de objeto.

Essas definições são ilustradas pela Fig. 2.13.

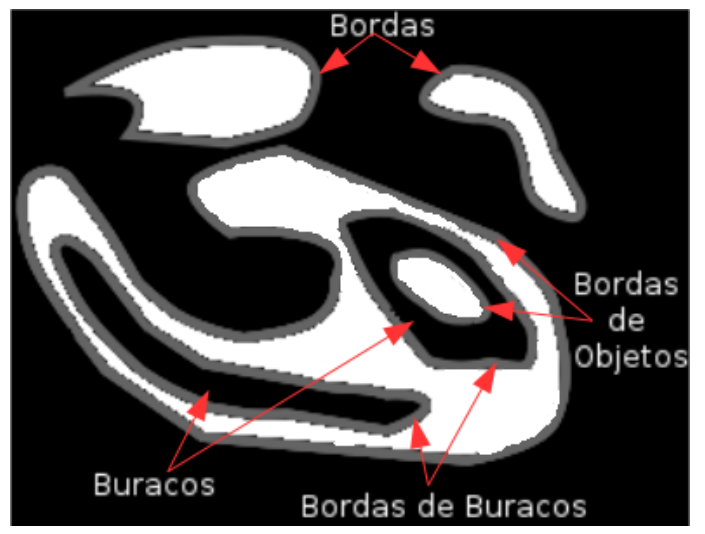

Fig. 2.13: Exemplo dos conceitos utilizados por Gray [Grä1] para a definição de imagens binárias no espaço continuo. 
Uma ilustração dos conceitos de borda, borda de objeto e borda de buraco é apresentada na Fig. 2.13, também são ilustrados os conceitos de objeto em branco e buraco em preto.

Dada uma imagem binária no espaço contínuo, a triangulação é uma operação onde linhas são desenhadas nas regiões de valor 1. Cada linha liga dois pontos (vértices) e cada ponto está em uma linha ou borda. A Fig. 2.14 exibe triangulações em uma imagem binária.

a)

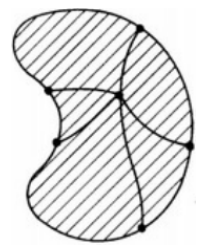

b)

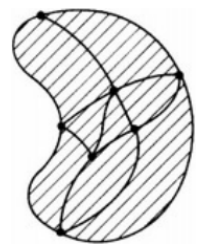

Fig. 2.14: Duas triangulações para uma imagem binária, onde a região hachurada indica um objeto. Imagem adaptada de [Gra71].

$\mathrm{Na}$ operação de triangulação, a intersecção de duas linhas ou de uma borda com uma linha é chamada vértice. Uma linha ou uma borda com um vértice em cada extremidade é uma aresta e uma curva fechada por arestas e vértices que possui valor 1 é chamada de região. Dadas as definições de aresta, vértice e região em uma triangulação, o número de vértices, o número de arestas e o número de regiões de uma triangulação são denotados por $v, e, r$ respectivamente, e o número de Euler é definido pela seguinte equação:

$$
E=v-e+r
$$

É conhecido em topologia que independente da triangulação e do número de lados de cada região (desde que cada região tenha pelo menos um lado), o número de Euler para um objeto conexo é sempre 1. O número de Euler para cada imagem na Fig. 2.14 pode ser calculado observando as quantidades $v, e, r$. Portanto, na imagem a) da Fig. 2.14 é verificado que: $v=6, e=10$ e $r=5$, $\operatorname{logo} E=6-5+5=1$, similarmente a imagem b) da Fig. 2.14 possui as quantidades $v=7, e=14$ e $r=8$, resultando em $E=7-14+8=1$.

Dada uma triangulação de um objeto conexo é possível adicionar um buraco ao mesmo, desenhando uma linha que liga dois vértices e atribuindo o valor 0 a região formada. Dessa forma, é possível calcular como o número de Euler varia de acordo com a inclusão do buraco, utilizando as variáveis $\Delta v, \Delta e, \Delta r$ e $\Delta E$ que indicam variação na quantidade de vértices, arestas, regiões e do número de Euler respectivamente, com a inclusão do buraco. Portanto, adicionando um buraco a um objeto conexo pelo método descrito acima, não são adicionados vértices, nem regiões (porque o buraco tem valor 0), mas é adicionado uma aresta, implicando no seguinte resultado:

$$
\begin{array}{r}
\Delta v=0, \Delta e=1, \Delta r=0 \\
\Delta E=\Delta v-\Delta e+\Delta r \\
\Delta E=0-1+0=-1 .
\end{array}
$$

Dados os resultados sobre o número de Euler, conclui-se que cada objeto conexo contribui com um "1" no valor do número de Euler e cada buraco contribui com "-1". Portanto, dado o número de objetos conexos $B$ e a quantidade de buracos $L$, o número de Euler $E$ pode ser calculado por $E=B-L$. A Fig. 2.15 exibe uma imagem formada por um componente conexo com buraco e sua triangulação. 


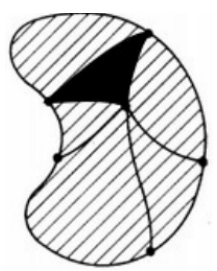

Fig. 2.15: Exemplo de triangulação de objeto conexo com buraco.

O número de vértices, o de arestas e o de regiões da imagem na Fig. 2.15 são $v=6, e=11$ e $r=5$, portanto, o número de Euler da imagem é $E=6-11+5=0$. O número de Euler da imagem também pode ser obtido utilizando o número de componentes conexos $B=1$ e de buracos $L=1$ que resulta em $E=1-1=0$.

A adaptação desse método de calcular o número de Euler para o espaço discreto é feito por meio de um mosaico (tessalation). Um mosaico de um plano denotado por $(p, q)$ consiste em $(q)$ $p$-gons regulares (polígonos de $p$ lados regulares) que se encontram em um ponto. É conhecido que no mosaico $(p, q)$, os $p$-gons se encontram com outros $p$-gons em $p$ pontos. É dito em [Gra71] que são conhecidos apenas três mosaicos dessa forma: o $(6,3)$ formado por hexagonais, o $(4,4)$ formado por quadrados e o $(3,6)$ formado por triângulos. O mosaico quadrado é particularmente interessante para a análise de imagens em computadores, porque é semelhante a interpretação dada aos pixels quando representados no computador. No mosaico quadrado, cada quadrado é um cell-bit e um cell-group é definido como os 4 cell-bits que se encontram em um ponto.

Os bit-quads são definidos como matrizes $2 \times 2$ de cell-bits. No mosaico quadrado existem apenas 16 bit-quads diferentes que podem ser divididos em 6 tipos diferentes: $Q 0, Q 1, Q 2, Q 3, Q 4$ e $Q D$, onde o número que segue a letra $Q$ indica a quantidade de cell-bits com valor "1", e $Q D$ são 2 bitquads que representam cell-bits de valor " 1 " na diagonal. Nota-se que existem 4 bit-quads diferentes para cada tipo $Q 1, Q 2$ e $Q 3$ que representam as rotações dos bit-cells. A Fig. 2.16 exibe os bit-quads definidos por Gray.

$$
\begin{aligned}
& Q 1=\begin{array}{ll}
1 & 0 \\
0 & 0
\end{array},, \begin{array}{ll}
0 & 1 \\
0 & 0
\end{array},, \begin{array}{ll}
0 & 0 \\
1 & 0
\end{array}, \begin{array}{ll}
0 & 0 \\
0 & 1
\end{array} \quad Q 2=\begin{array}{ll}
1 & 1 \\
0 & 0
\end{array},, \begin{array}{ll}
0 & 1 \\
0 & 1
\end{array},, \begin{array}{ll}
0 & 0 \\
1 & 1
\end{array},, \begin{array}{ll}
1 & 0 \\
1 & 0
\end{array}
\end{aligned}
$$

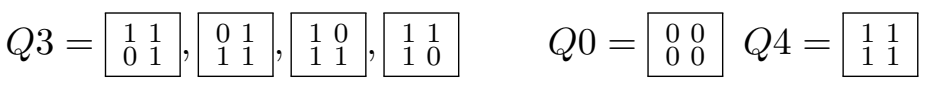

$$
\begin{aligned}
& Q D=\begin{array}{ll}
1 & 0 \\
0 & 1
\end{array}, \begin{array}{lll}
0 & 1 \\
1 & 0
\end{array}
\end{aligned}
$$

Fig. 2.16: Bit-quads definidos por Gray.

Para a realização do algoritmo de Gray é adotada uma triangulação em toda a área de valor "1" da imagem em quadrados unitários, adicionando linhas e vértices necessários. A partir dessa triangulação o número de vértices, arestas e regiões da imagem podem ser contados utilizando os bit-quads. A Fig. 2.17 exibe a triangulação utilizada no algoritmo e alguns bit-quads. 


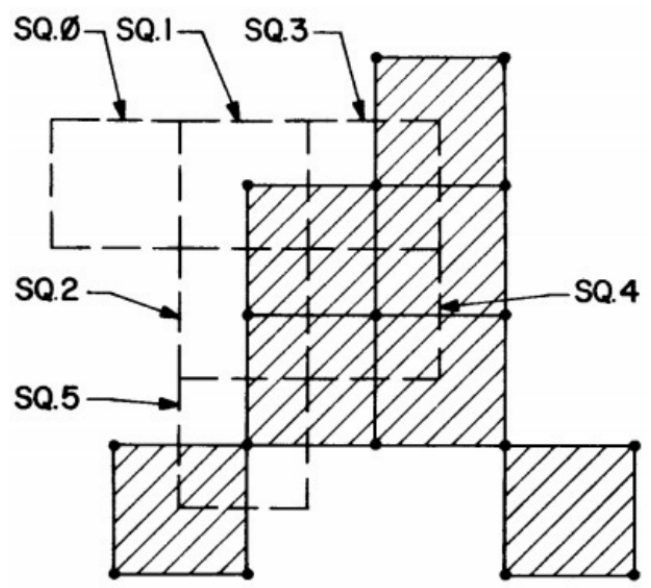

Fig. 2.17: Exemplo de uma triangulação de uma imagem binária e a utilização de bit-quads para contar vértices, arestas e regiões. Os quadrados hachurados indicam pixels de objeto e o exemplo SQ.5 é referente ao um bit-quad QD. Imagem retirada de [Gra71].

É possível notar na Fig. 2.17 que cada bit-cell do bit-quad representa 1 vértice, 2 metades de segmento de linha e $\frac{1}{4}$ de subárea. A contagem dos bit-quads de tipo $t$ é denotada por $n(t)$, onde $t$ é um tipo de bit-quad $(Q 1, Q 2, Q 3, Q 4, Q D)$. Equações para calcular o perímetro $p$ e a área $a$ podem ser formuladas como:

$$
\begin{aligned}
& a=\frac{n(Q 1)+2 n(Q 2)+2 n(Q D)+3 n(Q 3)+4 n(Q 4)}{4} \\
& p=n(Q 1)+n(Q 2)+2 n(Q D)+n(Q 3),
\end{aligned}
$$

a aproximação contínua da área $\hat{a}$ e do perímetro $\hat{p}$ podem ser obtidas pelas seguintes equações:

$$
\begin{aligned}
& \hat{a}=\frac{1}{4}\left[\frac{n(Q 1)}{2}+n(Q 2)+n(Q D)+\frac{7 n(Q 3)}{2}+4 n(Q 4)\right] \\
& \hat{p}=n(Q 2)+\frac{[n(Q 1)+n(Q 3)]}{\sqrt{2}} .
\end{aligned}
$$

A equação que resulta no número de Euler de uma imagem binária foi elaborada a partir dos números de vértices, arestas e regiões que cada bit-quad contribui na equação $E=v-e+r$, esses valores são denotados por $v^{\prime}, e^{\prime}$ e $r^{\prime}$ para vértices, arestas e regiões respectivamente, e são exibidos na Tab. 2.1

\begin{tabular}{|c|c|c|c|c|}
\hline $\begin{array}{c}\text { Tipo de } \\
\text { bit-quads }\end{array}$ & $v^{\prime}$ & $e^{\prime}$ & $r^{\prime}$ & $\left(v^{\prime}-e^{\prime}+r^{\prime}\right)$ \\
\hline$Q 0$ & 0 & 0 & 0 & 0 \\
\hline$Q 1$ & 1 & 1 & $1 / 4$ & $1 / 4$ \\
\hline$Q 2$ & 1 & $3 / 2$ & $1 / 2$ & 0 \\
\hline$Q 3$ & 1 & 2 & $3 / 4$ & $-1 / 4$ \\
\hline$Q 4$ & 1 & 2 & 1 & 0 \\
\hline$Q D$ & 1 & 2 & $1 / 2$ & $-1 / 2$ \\
\hline
\end{tabular}

Tab. 2.1: Tabela com a contribuição de cada bit-quad para o número de Euler.

Dadas as contribuições apresentadas na Tab. 2.1, obtém-se a equação para calcular o número de Euler de uma imagem binária usando os bit-quads:

$$
E=\sum\left(v^{\prime}-e^{\prime}+r\right)=\frac{n(Q 1)-n(Q 3)-2 n(Q 2)}{4} .
$$


Essa fórmula considera que os bit-quads do tipo $Q D$ estão conectados, ou seja, a imagem está sendo analisada com adajacência de 8-conectividade. Para analisar imagens com adjacência de 4conectividade, os cell-bits de $Q D$ com valor "1" devem ser desconexos, gerando assim um vértice a mais no bit-quad do $Q D$ conforme exibido na Fig. 2.18.

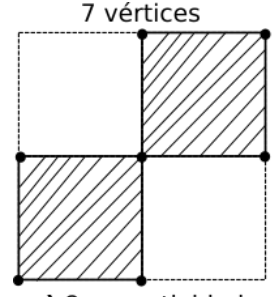

a) 8-conectividade

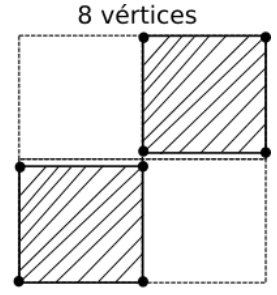

b) 4-conectividade

Fig. 2.18: Representação de um bit-quad de $Q D$ em uma adjacência de 8-conectividade com 8 vértices e em uma adjacência de 4-conectividade com 8 vértices.

A Fig. 2.18 mostra que mesmo ao acrescentar um vértice ao $Q D$ os números de arestas e regiões são mantidos em uma adjacência de 4-conectividade. Neste caso, a contribuição de $Q D$ no número de Euler passa a ser $+\frac{1}{2}$ e a equação para o cálculo do mesmo em imagens binárias com adjacência de 4-conectividade é:

$$
E=\sum\left(v^{\prime}-e^{\prime}+r\right)=\frac{n(Q 1)-n(Q 3)+2 n(Q 2)}{4} .
$$

\subsubsection{Contagem de número de buracos na árvore de componentes baseada em bit-quads}

Climent e Oliveira [CO14] desenvolveram um método incremental e eficiente para o cálculo do número de buracos na árvore de componentes que se baseia na contagem de bit-quads para o cálculo do número de Euler conforme proposta de Gray [Gra71]. Primeiramente os autores adaptam os conjuntos de bit-quads que calculam o número de Euler conforme proposta de Gray nos conjuntos $Q 1$ e $Q 2$ de quads (matrizes $2 \times 2$ com valores 1,0 e - ) para cada conectividade, mas diferentemente dos bit-quads do Gray, os índices dos quads de Climent e Oliveira não tem relação com o número de pixels ou cell-bits de objeto. Os quads de Climent e Oliveira para adjacência de 4-conectividade são exibidos abaixo:

$$
Q 1=\begin{array}{ll}
0 & - \\
1 & 0
\end{array}, \begin{array}{cc}
-0 & 0 \\
0 & 1
\end{array}, \begin{array}{ll}
0 & 1 \\
- & 0
\end{array},, \begin{array}{ll}
1 & 0 \\
0 & -
\end{array} \quad Q 2=\begin{array}{ll}
1 & 1 \\
0 & 1
\end{array}, \begin{array}{ll}
1 & 1 \\
1 & 0
\end{array}, \begin{array}{ll}
1 & 0 \\
1 & 1
\end{array}, \begin{array}{ll}
0 & 1 \\
1 & 1
\end{array} .
$$

e os quads para adjacência de 8-conectividade são exibidos abaixo:

$$
Q 1=\begin{array}{ll}
0 & 0 \\
1 & 0
\end{array}, \begin{array}{ll}
0 & 0 \\
0 & 1
\end{array}, \begin{array}{ll}
0 & 1 \\
0 & 0
\end{array}, \begin{array}{ll}
1 & 0 \\
0 & 0
\end{array} \quad Q 2=\begin{array}{ll}
1 & - \\
0 & 1
\end{array}, \begin{array}{ll}
-1 \\
1 & 0
\end{array}, \begin{array}{ll}
1 & 0 \\
- & 1
\end{array},, \begin{array}{ll}
0 & 1 \\
1 & -
\end{array} .
$$

O símbolo "-" nos quads de Climent e Oliveira indica que aquela posição pode conter qualquer valor. O cálculo do número de Euler para esses quads (em ambas conectividades) é dado por:

$$
E=\frac{n(Q 1)-n(Q 2)}{4} .
$$

Essa equação é válida, porque os bit-quads de $Q D$ contribuem com -2 unidades para a equação do número de Euler em adjacência de 8-conectividade proposta por Gray. Essa contribuição se mantém através dos quads de $Q 2$ de Climent e Oliveira, porque quando encontrado um bit-quad de $Q D$, existirá dois quads do tipo $Q 2$ de Climent e Oliveira, por exemplo, se o bit-quad $\begin{array}{ll}1 & 0 \\ 0 & 1\end{array}$ é encontrado, então os quads $\begin{array}{ll}1 & - \\ 0 & 1\end{array}$ e $\begin{array}{cc}1 & 0 \\ -1 & 1\end{array}$ também serão encontrados, mantendo assim a contribuição de -2 unidades do $Q D$ na equação do número de Euler do Gray. Semelhantemente para a equação 
de 4-conectividade, é utilizado os quads de $Q 1$ de Climent e Oliveira para manter a contribuição de +2 unidades dos bit-quads de $Q D$ do Gray, por exemplo, caso a imagem tenha o bit-quad $\begin{array}{cc}1 & 0 \\ 0 & 1\end{array}$, então ela também tem os quads \begin{tabular}{cc|cc}
- & 0 \\
0 & 1
\end{tabular} e $\begin{array}{cc}1 & 0 \\
0 & -\end{array}$, mantendo a contribuição de +2 unidades. Os quads de $Q 1$ de Climent e Oliveira ainda mantém a contagem dos bit-quads de $Q 1$ de Gray e os quads de Q2 mantém a contagem de $Q 3$ do Gray.

Para o cálculo do número de buracos na árvore de componentes de uma imagem em níveis de cinza, Climent e Oliveira propõe padrões que comparam níveis de cinza baseados nos quads. Os padrões e exemplos são baseados na árvore de componentes dos conjuntos de níveis superiores (e sua max-tree), mas utilizando operações duais ou invertendo a imagem, o método pode ser utilizado na árvore de componentes dos conjuntos de níveis inferiores (e sua min-tree). Cada padrão da árvore de componentes é representado pela letra $p$ que indica o pixel analisado e mais 3 símbolos que podem ser as letras $L, l, g, G$ ou - , tal que, $f(L)<f(p), f(l) \leq f(p), f(g) \geq f(p), f(G)>f(p)$ e - que pode assumir qualquer valor. Os padrões para serem utilizados na árvore de componentes de 4-conectividade foram definidos como:

$$
\begin{array}{r}
Q 1=\begin{array}{ll}
L & - \\
p & L
\end{array},, \begin{array}{ll}
-\bar{L} \\
L & p
\end{array}, \begin{array}{ll}
L & p \\
- & L
\end{array},, \begin{array}{ll}
p & L \\
L & -
\end{array} \quad Q 2=\begin{array}{ll}
G & p \\
L & G
\end{array},, \begin{array}{ll}
p & G \\
G & L
\end{array}, \begin{array}{ll}
G & L \\
p & G
\end{array},, \begin{array}{ll}
L & G \\
G & p
\end{array}, \\
\begin{array}{ll}
p & g \\
L & G
\end{array}, \begin{array}{ll}
g & p \\
G & L
\end{array}, \begin{array}{ll}
G & L \\
g & p
\end{array}, \begin{array}{ll}
L & G \\
p & g
\end{array}, \\
\begin{array}{ll}
g & g \\
L & p
\end{array},, \begin{array}{ll}
g & g \\
p & L
\end{array}, \begin{array}{ll}
p & L \\
g & g
\end{array}, \begin{array}{ll}
L & p \\
g & g
\end{array},
\end{array}
$$

similarmente foram definidos padrões dos tipos $Q 1$ e $Q 2$ para árvore de componentes de adjacência de 8-conectividade, conforme exibido abaixo:

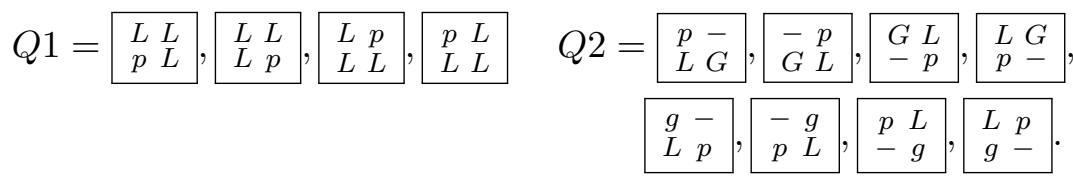

Climent e Oliveira concluem que os padrões da árvore de componentes de $Q 1$ são equivalentes aos quads binários de $Q 1$ para suas respectivas conectividades, porque os pixels do tipo $L$ sempre representam pixels de valor 0 na imagem binária do componente conexo de nível $f(p)$ e $p$ sempre estará no componente conexo de nível $f(p)$. Os padrões de $Q 2$ da árvore de componentes também são equivalentes aos quads binários de $Q 2$, porque os pixels de tipo $G$ e tipo $g$ estão no componente conexo de nível $f(p)$. Os padrões da árvore de componentes utilizam os pixels $G$ para não contar quads repetidos ao variar o pixel de referência (pixel $p$ nos padrões). A Fig. 2.19 exibe um exemplo onde poderia existir contagem de padrões de maneira incorreta se não fosse o uso dos pixels de tipo $G$.

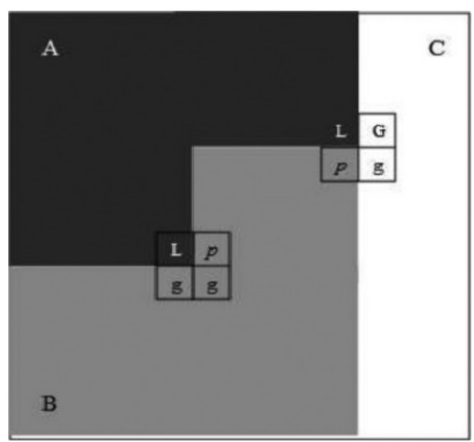

Fig. 2.19: Exemplo que demonstra padrões que precisam utilizar pixels do tipo G para não obter contagem incorreta. Imagem retirada de [CO14]

Na Fig. 2.19, dois quads não são contados para o mesmo conjunto de pixels apenas por causa 
do pixel do tipo $G$ nos padrões. Caso o pixel do tipo $G$ no padrão $\begin{aligned} & \underset{p}{\operatorname{L}} G \\ & g\end{aligned}$ fosse trocado por um pixel do tipo $g$ (pixel de objeto na árvore de componentes), o conjunto de pixels que contém o padrão \begin{tabular}{|l|l|l|l|l|l}
\hline & $p$ \\
$g$ & $g$
\end{tabular} do $Q 2$, também conteria $\begin{array}{ll}L & g \\
p & g\end{array}$ do $Q 2$, contando assim duas vezes o mesmo quad.

As quantidades de quads são atributos incrementais, mas diferentemente da área o procedimento de juntar os atributos calculados nos nós filhos com o atributo calculado no nó pai não é simplesmente uma soma. Embora alguns casos uma simples soma resulte na contagem correta, existem casos em que o quad contado no nó filho não existe no nó pai, conforme exemplo exibido na Fig. 2.20.

a)

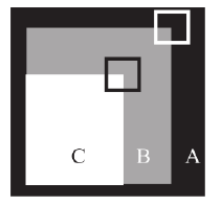

b)

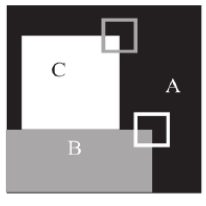

Fig. 2.20: Exemplo da utilização de soma para juntar as contagens de quads dos nós filhos com o nó pai. Na imagem a), o quad de $Q 1$ contando nó C não existe em seu nó pai (nó B). Na imagem b), a soma das contagens é o suficiente para juntar as mesmas. Imagem retirada de [CO14].

Na Fig. 2.20 a imagem a) mostra um exemplo em que um quad contado em um nó não está presente em seu nó pai, e na imagem b) a soma da contagem do nó pai com a contagem de seu nó filho produz o resultado esperado. Para resolver esse problema Climent e Oliveira propõem os padrões dos tipos $Q 3$ e $Q 4$ que removem os quads que foram contados nos nós filhos, mas que não existem no nó pai. Os padrões para 4-conectividade são exibidos abaixo:

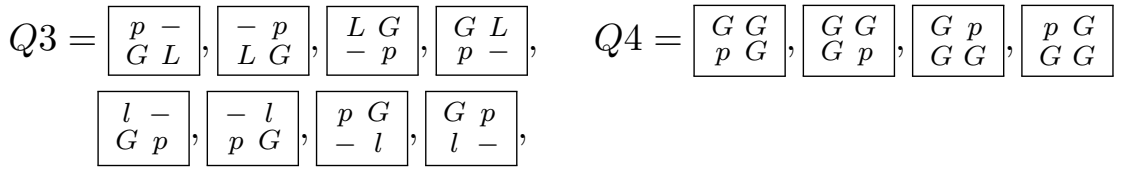

para 8-conectividade foram propostos os seguintes padrões:

$Q 3=\begin{array}{ll}L & p \\ G & L\end{array}, \begin{array}{ll}p & L \\ L & G\end{array}, \begin{array}{ll}L & G \\ p & L\end{array},, \begin{array}{ll}G & L \\ L & p\end{array}$,
$\begin{array}{ll}p & l \\ G & L\end{array}, \begin{array}{ll}l & p \\ L & G\end{array}, \begin{array}{ll}L & G \\ l & p\end{array}, \begin{array}{ll}G & L \\ p & l\end{array}$
$\begin{array}{ll}l & l \\ G & p\end{array}, \begin{array}{ll}l & l \\ p & G\end{array}, \begin{array}{ll}p & G \\ l & l\end{array},, \begin{array}{ll}G & p \\ l & l\end{array}$.

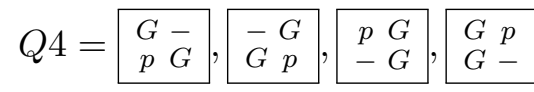

Definidos todos os padrões e quads, Climent e Oliveira propõem quatro procedimentos que devem ser executados no algoritmo da construção da árvore de componentes (max-tree) descrita por Salembier et. al. [SOG98]. Os procedimentos são: NEW-NoDE executado quando um novo nó é criado, ADD-To-Node executado quando um novo pixel de um nó já existente é encontrado, MERGE-NodE executado quando a relação de parentesco entre dois nós é criada e GET-ATTRIBUTE-VALue procedimento que calcula o número de buracos. Os procedimentos são descritos nos Algs. 5, 6, 7 e 8 . 

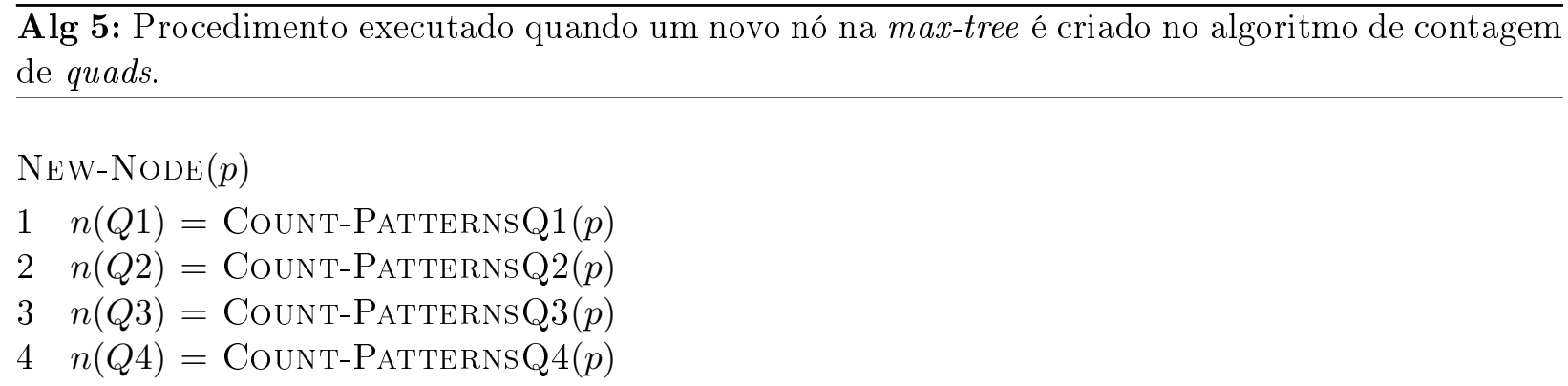

No Alg. 5, quando um novo nó é criado através de um pixel $p$ são contabilizados todos os quads com referência ao pixel p utilizando os padrões definidos por Climent e Oliveira. As funções Count-PATtERnsQI contam os padrões do tipo indicado por QI no sufixo do nome de cada função. O procedimento ADD-To-NodE é exibido pelo Alg. 6.

Alg 6: Procedimento executado quando um pixel é adicionado a um nó existente da max-tree no algoritmo de contagem de quads.

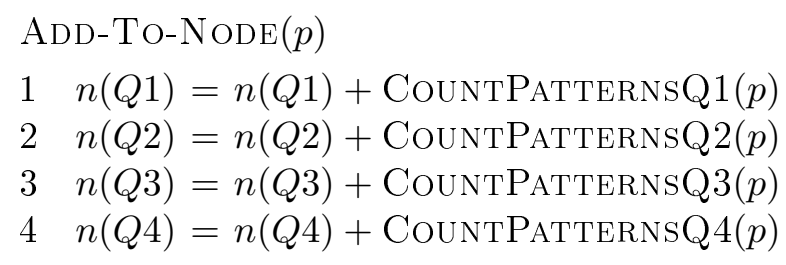

No Alg. 6, a contagem de quads é acumulada em seu respectivo contador. O procedimento Merge-Nodes é descrito no Alg. 7.

Alg 7: Procedimento executado quando uma relação de parentesco é criada na max-tree no algoritmo de contagem de quads.

\section{$\operatorname{Merge-Nodes}(p)$}

$1 \quad n(Q 1)=n(Q 1)+\operatorname{child}_{n(Q 1)}-n(Q 3)$

$2 n(Q 2)=n(Q 2)+\operatorname{child}_{n(Q 2)}-n(Q 4)$

No Alg. 7, as contagens de quads dos nós filhos $\left(\operatorname{child}_{n(Q 1)}\right.$ e $\left.\operatorname{child}_{n(Q 2)}\right)$ são juntadas (somadas) com as do nó pai, e os quads dos nós filhos que não existem no nó pai são removidos através de subtrações dos valores de $n(Q 3)$ e $n(Q 4)$. O procedimento GeT-Attribute-Value é exibido em 8.

Alg 8: Procedimento que computa o número de buracos a partir da contagem de quads de um nó da max-tree.

Get-Attribute-Value()

1 return $1-\frac{n(Q 1)-n(Q 2)}{4}$

O Alg. 8 computa o número de buracos utilizando o fato que cada nó da árvore de componentes representa apenas um componente conexo e a conhecida relação entre número de componentes conexos e o número de Euler para obter o número de buracos. Exemplos de padrões contados em 
nós da árvore de componentes são exibidos na Fig. 2.21 e um exemplo de execução dos algoritmos é exibido a seguir.

a)

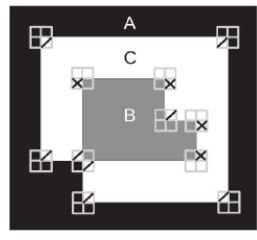

b)

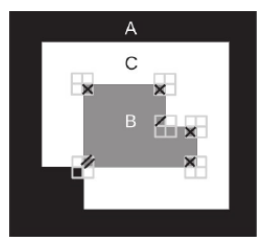

c)

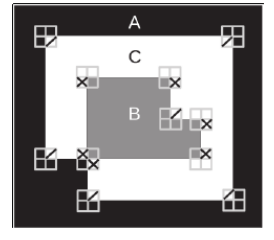

d)

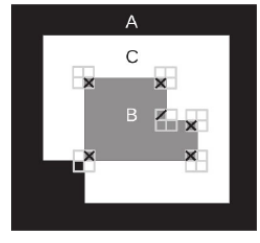

Fig. 2.21: Exemplos de quads nos nós de uma árvore de componentes. Imagens retiradas de [CO14].

A Fig. 2.21 exibe quatro imagens nomeadas: a), b), c), d). As imagens a) e b) exibem as contagens dos padrões de 4-conectividade e as imagens c) e d) exibem as contagens dos padrões de 8-conectividade. A imagem a) exibe as contagens dos padrões do nó $A$ que possui 8 padrões do $Q 1$ marcados com um traço e 4 padrões do $Q 2$ marcados com dois traços, portanto, a quantidade de buracos do nó $A$ pode ser calculada por $1-((8-4) / 4)=0$. Na imagem b) existem 3 padrões do $Q 3$ marcados com um traço (existe uma marcação com dois traços para indicar que existem 2 bit-quads $Q 3$ contados) e 4 padrões do $Q 4$ marcados com dois traços cruzados e 1 bit-quad $Q 2$ não marcado. O nó $B$ é nó pai do nó $A$ na max-tree, portanto, o nó $B$ acumula os valores das contagens, resultando em $n(Q 1)=0+8-3=5\left(8\right.$ do $\operatorname{child}_{n(Q 1)}$ e -3 de $\left.n(Q 3)\right)$ e $n(Q 2)=1+4-4=1(1$ da contagem do próprio nó, 4 de $\left.\operatorname{child}_{n(Q 2)}\right)$ e -4 de $\left.n(Q 4)\right)$. A partir das contagens dos quads, o número de buracos do nó $B$ é calculado por: $1-((5-1) / 4)=0$.

As imagens c) e d) na Fig. 2.21 apresentam as contagens de padrões de 8-conectividade. A imagem c) possui 6 padrões do $Q 1$ marcados com um traço e 6 padrões do $Q 2$ marcados com dois traços cruzados no nó $A$, o número de buracos do nó $A$ pela equação: $1-((6-6) / 4)=1$. Na imagem d) são encontrados: 1 padrão do $Q 3$ marcado com um traço e 5 padrões do $Q 4$ marcados com dois traços cruzados. O nó $A$ é filho do nó $B$ na max-tree, portanto, as contagens dos quads em $B$ são dadas por: $n(Q 1)=0+6-1=5\left(6\right.$ de $\operatorname{child}_{n(Q 1)}$ e -1 de $\left.n(Q 3)\right)$ e $n(Q 2)=0+6-5=1$ (6 de $\operatorname{child}_{n(Q 2)}$ e -5 de $\left.n(Q 4)\right)$. A partir destas contagens, o número de buracos do nó $B$ é dado por: $1-((5-1) / 4)=0$.

Climent e Oliveira utiliza o número de buracos para filtragem e segmentação de placas de carros e o algoritmo de contagem de quads proposto pelos mesmos é usado como base para a algoritmo proposto nesse trabalho. 


\section{Capítulo 3}

\section{Método proposto}

O trabalho tem como objetivo estender e melhorar o método de contagem de quads na árvore de componentes proposto por Climent e Oliveira [CO14]. Essa extensão se baseia nos quads e padrões de Climent e Olivera para propor novos conjuntos de quads e padrões que possibilite o cálculo de todos os atributos calculáveis pela contagem de bit-quads apresentados no trabalho de Gray [Gra71], incluindo: número de Euler (número de buracos), área, perímetro e as aproximações contínuas dos dois últimos. O capítulo apresentará as formulações, exemplos e a prova de corretude na árvore de componentes dos conjuntos de níveis superiores e a max-tree, porém, utilizando operações duais ou invertendo a imagem as mesmas formulações, exemplos e prova de corretude, também são aplicáveis na árvore de componentes dos conjuntos de níveis inferiores e da min-tree.

\subsection{Definições}

\subsubsection{Extensão dos quads para contagem de todos os bit-quads}

Seguindo a nomenclatura de Climent e Oliveira [CO14], nesta seção, são definidos os quads do método proposto neste trabalho. Um quad $Q$ é definido como uma função $Q: D_{Q} \rightarrow S_{B}$, onde $D_{Q} \subset \mathbb{Z}^{2}$ é o domínio do quad e o contradomínio $S_{Q}=\{0,1, o,-\}$ é um conjunto de símbolos para serem utilizados em imagens binárias. Um quad $Q$ é representado por um quadrado que contém uma origem $o=(0,0)$ e mais três símbolos. O domínio $D_{Q}$ é obtido a partir da posição relativa a origem de cada elemento do domínio no quadrado, tal que, para um ponto $(x, y)$ à esquerda da origem implica em $x=-1$, a direita implica em $x=1$ acima da origem implica em $y=-1$ e abaixo da origem implica em $y=1$, por exemplo, o quad $Q=\begin{array}{lll}o & s_{1} \\ s_{2} & s_{3}\end{array}$ possui domínio $D_{Q}=\{(0,0),(1,0),(0,1),(1,1)\}$ e os valores da função para cada elemento do domínio são: $Q((0,0))=o, Q((1,0))=s_{1}, Q((0,1))=s_{2}$ e $Q((1,1))=s_{3}$, similarmente o quad $Q^{\prime}=\begin{array}{cc}s_{1}^{\prime} & s_{2}^{\prime} \\ s_{3}^{\prime} & o\end{array}$ possui o domínio $D_{Q^{\prime}}=\{(0,0),(-1,-1),(0,-1),(-1,0)\}$ e os seguintes valores para cada elemento do domínio: $Q^{\prime}((0,0))=o, Q^{\prime}((-1,-1))=s_{1}^{\prime}, Q^{\prime}((0,-1))=s_{2}^{\prime}$ e $Q^{\prime}((-1,0))=s_{3}^{\prime}$. Para realizar uma bijeção aos bit-quads apresentados por Gray [Gra71], os quads propostos são separados em conjuntos de acordo com a quantidade de símbolos 1 e $o$ que possui, conforme exibido abaixo

$$
\begin{aligned}
& Q_{1}=\left\{\begin{array}{ll}
o & 0 \\
0 & 0
\end{array},, \begin{array}{ll}
0 & 0 \\
0 & 0
\end{array},, \begin{array}{ll}
0 & 0 \\
0 & 0
\end{array},, \begin{array}{ll}
0 & 0 \\
0 & o
\end{array}\right\}, \quad Q_{2}=\left\{\begin{array}{ll}
o & 1 \\
0 & 0
\end{array}, \begin{array}{ll}
0 & o \\
0 & 1
\end{array}, \begin{array}{ll}
0 & 0 \\
1 & o
\end{array},, \begin{array}{ll}
1 & 0 \\
0 & 0
\end{array}\right\}, \\
& Q_{3}=\left\{\begin{array}{ll}
1 & 1 \\
0 & o
\end{array}, \begin{array}{ll}
0 & o \\
1 & 1
\end{array},, \begin{array}{ll}
o & 0 \\
1 & 1
\end{array},, \begin{array}{ll}
1 & 1 \\
0 & 0
\end{array}\right\}, \quad Q_{4}=\left\{\begin{array}{ll}
o & 1 \\
1 & 1
\end{array}\right\}, Q_{D}=\left\{\begin{array}{ll}
0 & 0 \\
0 & 1
\end{array},, \begin{array}{ll}
0 & 1 \\
0 & 0
\end{array}\right\} .
\end{aligned}
$$

Os quads do tipo $Q_{D}$ não possuem elementos conexos em uma relação de adjacência de 4conectividade (em relação aos símbolos que representam pixels de objeto), por outro lado, os nós de uma árvore de componentes são componentes conexos. Neste sentido, os quads de $Q_{D}$ precisam ser adaptados para a criação de padrões correspondentes que possam ser utilizados na árvore de componentes de adjacência de 4-conectividade. Portanto, no método proposto, quando a computação é feita em um contexto de adjacência de 4-conectividade, é considerado que existe um espaço que 
separa os dois símbolos que representam pixels de objeto nos quads de $Q_{D}$, esses pixels (símbolos) separados por um pequeno espaço nos quads de $Q_{D}$ formam dois quads do tipo $Q_{1}$ (um para cada pixel) e para contar esses quads é definido o seguinte conjunto de quads:

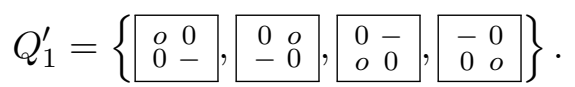

Dado que $n(t)$ representa a quantidade de quads do tipo $t$, as contagens de quads dos tipos $Q_{1}$ e $Q_{D}$ da relação de adjacência 8-conectividade podem ser relacionadas com a contagem dos quads de $Q_{1}^{\prime}$ pela seguinte equação:

$$
n\left(Q_{1}^{\prime}\right)=n\left(Q_{1}\right)+2 n\left(Q_{D}\right) .
$$

Por simplicidade, as formulações e exemplos seguintes utilizam apenas os quads de relação de adjacência 8-conectividade, porém, os mesmos podem ser adaptados para os quads de relação de adjacência de 4-conectividade.

A partir dos conjuntos de quads definidos nesta seção, é definida a família de conjuntos de quads como: $\mathcal{Q}=\left\{Q_{1}, Q_{2}, Q_{3}, Q_{4}, Q_{D}\right\}$.

Seja $\mathcal{G}=\{g: \mathcal{D} \rightarrow\{0,1\}\}$ o conjunto de todas as imagens binárias e $\mathcal{P}(\mathcal{D})$ sua notação em conjunto, é definido o conjunto de matching para a tripla $\left(X, p, Q_{i}\right) \in \mathcal{P}(\mathcal{D}) \times \mathcal{D} \times \mathcal{Q}$ da seguinte maneira:

$$
\begin{aligned}
& M_{Q_{i}, X}(p)=\left\{Q \in Q_{i}: \forall x \in D_{Q},\right. Q(x)=0 \Rightarrow x+p \notin X \text { and } \\
&Q(x)=1 \Rightarrow x+p \in X\} .
\end{aligned}
$$

É dito que um quad $Q$ foi contado, se existe um conjunto de matching $M_{Q_{i}, X}(p)$, tal que, $Q \in$ $M_{Q_{i}, X}(p)$. A partir do conjunto de matching é definida a função de contagem de quads para a tripla $\left(X, p, Q_{i}\right) \in \mathcal{P}(\mathcal{D}) \times \mathcal{D} \times \mathcal{Q}$ da seguinte maneira:

$$
\operatorname{count} B\left(p, Q_{i}, X\right)=\left|M_{Q_{i}, X}(p)\right| .
$$

Um quad $Q$ é contado pela função $\operatorname{count} B\left(p, Q_{i}, X\right)$, se $Q \in M_{Q_{i}, X}(p)$ e o mesmo quad não é contado pela função count $B\left(p, Q_{i}, X\right)$, se $Q \notin M_{Q_{i}, X}(p)$.

A função count $B$ pode ser vista como translações dos quads, posicionando-os com a origem no pixel $p$ e contando aqueles quads cujos pixels vizinhos de $p$ de acordo com o domínio do quad transladado tenha o mesmo valor que o seu elemento correspondente no quad. A Fig. 3.1 exibe graficamente as translações de dois quads e as comparações de valores para verificar se o quad foi contado.
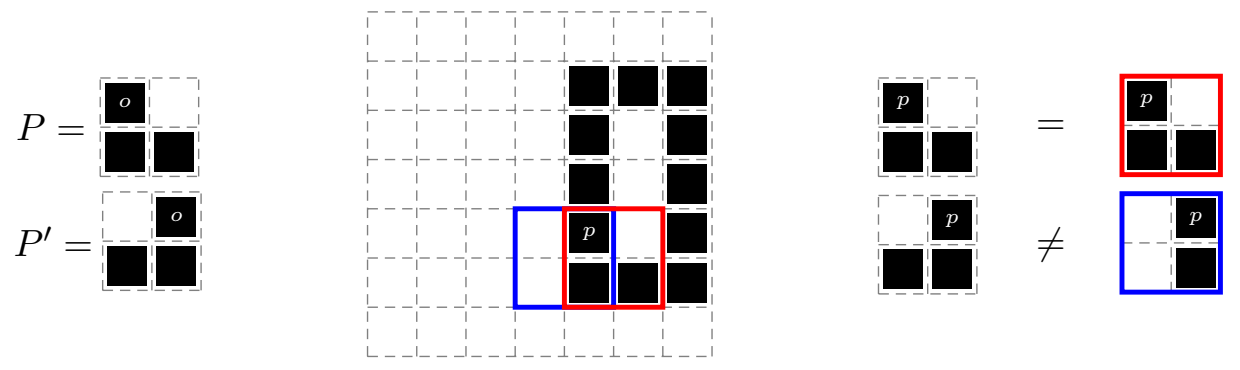

Fig. 3.1: Exemplo de translações dos quads $Q$ (em vermelho) e $Q^{\prime}$ (em azul) e as comparaçôes feitas nas regiões da imagem definidas pelos domínios dos quads.

Conforme pode ser observado na Fig. 3.1, o quad $Q \in Q_{3}$ (em vermelho) possui o domínio: $D_{Q}=$ $\{(0,0),(1,0),(0,1),(1,1)\}$, o mesmo transladado por $p=(4,4)$ é $D_{Q}+p=\{(4,4),(5,4),(4,5),(5,5)\}$ e então são verificadas as seguintes condições (comparações): $(i) Q((1,0))=0 \Rightarrow(5,4) \notin X$, $($ ii $) Q((0,1))=1 \Rightarrow(4,5) \in X$ e $($ iii $) Q((1,1))=1 \Rightarrow(5,5) \in X$, e como todas são verdadeiras $Q \in M_{Q_{3}, X}(p)$ e é dito que $Q$ é contado por count $B\left(p, Q_{i}, X\right)$. O quad $Q^{\prime} \in Q_{3}$ (em azul) possui o seguinte domínio: $D_{Q^{\prime}}=\{(0,0),(0,1),(-1,1),(-1,0)\}$, o mesmo transladado por $p=(4,4)$ re- 
sulta em: $D_{Q^{\prime}}+p=\{(4,4),(4,5),(3,5),(3,4)\}$ e então as seguintes condições (comparações) são verificadas: $\left(i^{\prime}\right) Q^{\prime}(0,1)=1 \Rightarrow(4,5) \in X,\left(i i^{\prime}\right) Q^{\prime}(-1,1)=1 \Rightarrow(3,5) \in X$ e $\left(i i i^{\prime}\right) Q^{\prime}(-1,0)=0 \Rightarrow$ $(3,4) \notin X$, neste caso, a condição $\left(i i^{\prime}\right)$ não é satisfeita, portanto, $Q^{\prime} \notin M_{Q_{3}, X}(p)$ e é dito que $Q^{\prime}$ não é contado por count $B\left(p, Q_{3}, X\right)$.

Geralmente deseja-se contar a quantidade de quads encontrados em uma imagem binária para cada conjunto de $\mathcal{Q}$. Neste sentido, é definido o atributo $\kappa_{Q_{i}}$ para uma imagem binária $X$ e $i \in$ $\{1,2,3,4, D\}$ da seguinte forma:

$$
\kappa_{Q_{i}}(X)=\sum_{p \in X} \operatorname{count} B\left(p, Q_{i}, X\right) .
$$

A Fig. 3.2 exibe a translação de cada quad encontrado em uma imagem binária de exemplo.

a)

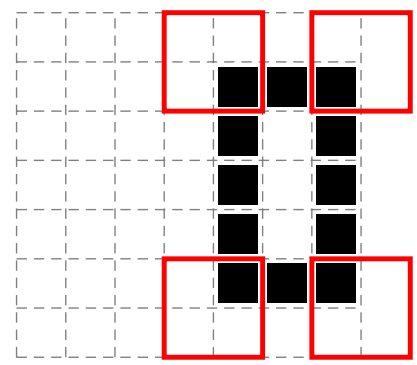

b)

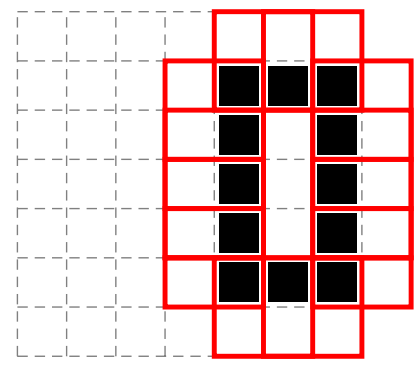

c)

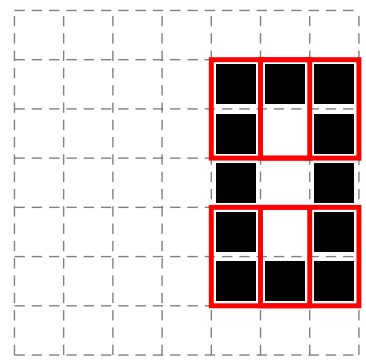

Fig. 3.2: Domínios dos quads contados em uma imagem binária. Os domínios dos quads de $Q_{1}$ são exibidos na imagem a), os dominios dos quads de $Q_{2}$ são exibidos na imagem $\boldsymbol{b}$ ) e os de $Q_{3}$ na imagem $\boldsymbol{c}$ ).

A Fig. 3.2 representa o domínio transladado de cada quad contado na imagem binária de exemplo. O cálculo da quantidade de quads da imagem de exemplo exibida na Fig. 3.2 resulta em: $\kappa_{Q_{1}}(X)=4, \kappa_{Q_{2}}(X)=12, \kappa_{Q_{3}}(X)=4, \kappa_{Q_{4}}(X)=\kappa_{Q_{D}}(X)=0$.

\subsubsection{Extensão dos padrões de Climent e Oliveira para contagem de todos os quads na árvore de componentes}

Para a contagem de todos os 5 tipos de quads na árvore de componentes eficientemente, é proposto uma extensão dos padrões apresentados por Climent e Oliveira [CO14]. Essa extensão acrescenta novos tipos de padrões aos conjuntos de padrões definidos por Climent e Oliveira, permitindo assim contar todos os tipos de quads definidos na Sec. 3.1.1 e consequentemente encontrar o número de bit-quads definidos por Gray [Gra71] em todos os nós de uma árvore de componentes.

Seja $S=\{o, L, l, G, g,-\}$ um conjunto de símbolos que representam relações de ordem de intensidade dos pixels de uma imagem utilizado para definir o conjunto de matching de padrões, conforme será apresentado mais a frente nesta seção. Um padrão $P$ é uma função que mapeia um domínio $D_{P}$ ao conjunto de símbolos $S$, onde o domínio do padrão é definido de maneira similar ao domínio de quads (veja Sec . 3.1.1). Esses padrões são organizados em conjuntos conforme mostrado abaixo:

$$
\begin{aligned}
& P_{1}=\left\{\begin{array}{ll}
L & L \\
o & L
\end{array},, \begin{array}{ll}
L & L \\
L & o
\end{array},, \begin{array}{ll}
L & 0 \\
L & L
\end{array},, \begin{array}{ll}
o & L \\
L & L
\end{array}\right\}
\end{aligned}
$$

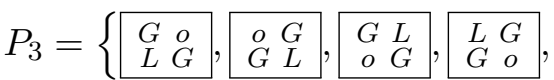

$$
\begin{aligned}
& \begin{array}{l}
\begin{array}{ll}
o & g \\
L & G
\end{array}, \begin{array}{ll}
g & o \\
G & L
\end{array},, \begin{array}{ll}
G & L \\
g & o
\end{array},, \begin{array}{ll}
L & G \\
o & g
\end{array}, \\
\begin{array}{ll}
g & g \\
L & o
\end{array},, \begin{array}{ll}
g & g \\
o & L
\end{array},\left[\begin{array}{ll}
o & L \\
g & g
\end{array},, \begin{array}{ll}
L & 0 \\
g & g
\end{array}\right\}
\end{array}
\end{aligned}
$$

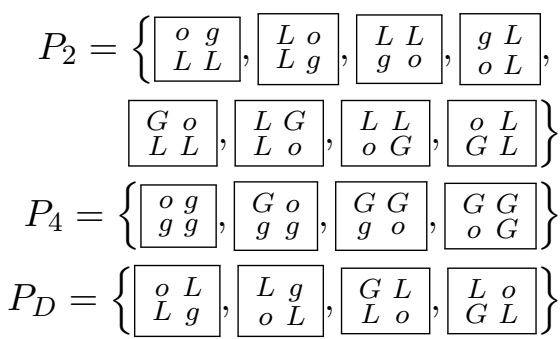

A partir dos conjuntos de padrões definidos nesta seção, a familía de conjuntos de padrões é definida como: $\mathbb{P}=\left\{P_{1}, P_{2}, P_{3}, P_{4}, P_{D}\right\}$. 
Seja $\mathcal{F}=\{f: \mathcal{D} \rightarrow \mathbb{K}\}$ o conjunto de todas as imagens de domínio $\mathcal{D}$ e contradomínio $\mathbb{K}$, o conjunto de matching de padrões para a tripla $\left(p, P_{i}, f\right) \in \mathcal{D} \times \mathbb{P} \times \mathcal{F}$ é definido por:

$$
\begin{aligned}
\mathcal{M}_{f, P_{i}}(p)=\left\{P \in P_{i}: \forall x \in D_{P},\right. & (P(x)=l \Rightarrow f(x+p) \leq f(p) \text { and } \\
& P(x)=L \Rightarrow f(x+p)<f(p) \text { and } \\
& P(x)=g \Rightarrow f(x+p) \geq f(p) \text { and } \\
& P(x)=G \Rightarrow f(x+p)>f(p))\}
\end{aligned}
$$

A partir do conjunto de matching de padrões, a função count $: \mathcal{D} \times \mathbb{P} \times \mathcal{F} \rightarrow \mathbb{Z}$ é definida para uma tripla $\left(p, P_{i}, f\right) \in \mathcal{D} \times \mathbb{P} \times \mathcal{F}$ como:

$$
\operatorname{count}\left(p, P_{i}, f\right)=\left|\mathcal{M}_{P_{i}, f}(p)\right| \text {. }
$$

É dito que um padrão $P$ qualquer é contado se existe um conjunto de matching de padrões que o contém, ou seja, $\exists \mathcal{M}_{P_{i}, f}(p)$, tal que, $P \in \mathcal{M}_{P_{i}, f}(p)$. A função count $\left(p, P_{i}, f\right)$ conta um padrão $P$, se $P \in \mathcal{M}_{P_{i}, f}(p)$ e ela não conta o padrão $P$, se $P \notin \mathcal{M}_{P_{i}, f}(p)$. A função count pode ser vista como a contagem de padrões transladados por $p$ (origem posicionada em $p$ ), tal que, as relações de ordem definidas pelos símbolos do padrão são obedecidas pelos pixels da imagem na vizinhança definida pelo domínio do padrão transladado. A verificação se dois padrões estão no conjunto de matching de padrões é exibida na Fig. 3.3.
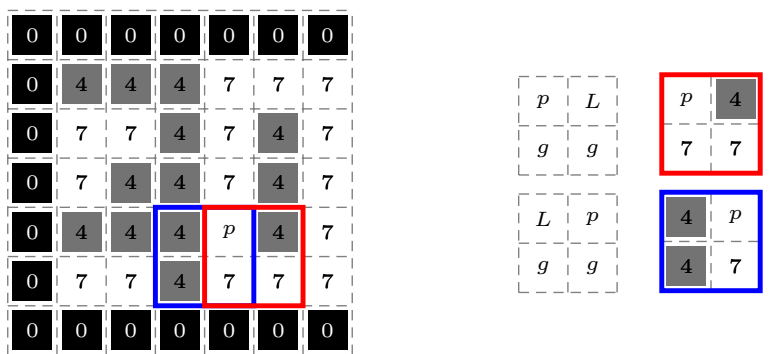

Fig. 3.3: Translações de dois padrões $P$ e $P^{\prime}$ por um pixel $p$ e a verificação de pixels nas vizinhanças definidas pelos dominios dos padrões transladados.

A Fig. 3.3 exibe translações de dois padrões $P, P^{\prime} \in P_{3}$ por um pixel $p$ em uma imagem em nível de cinza $f: \mathcal{D} \rightarrow\{0,1, \ldots, 7\}$. Na Fig. 3.3, o padrão $P$ tem o domínio $D_{P}=\{(0,0)$, $(1,0),(0,1),(1,1)\}$ que transladado por $p=(4,4)$ resulta em: $D_{P}+p=\{(4,4),(5,4),(4,5)$, $(5,5)\}$. Para verificar se $P$ está no conjunto de matching da função $\operatorname{count}\left(p, P_{3}, f\right)$ são verificados os seguintes casos: $(i) P((1,0))=L \Rightarrow f(5,4)<f(4,4),($ ii $) P((0,1))=g \Rightarrow f(4,5) \geq$ $f(4,4)$ e $($ iii $) P((1,1))=g \Rightarrow f(5,5) \geq f(4,4)$ e como todos os casos são verdadeiros, então $P \in \mathcal{M}_{P_{3}, f}(p)$ e é dito que $P$ foi contado por $\operatorname{count}\left(p, P_{3}, f\right)$. O padrão $P^{\prime}$ possui o seguinte domínio $D_{P^{\prime}}=\{(0,0),(0,1),(-1,1),(-1,0)\}$ que translado por $p=(4,4)$ resulta em: $D_{P^{\prime}}+p=\{(4,4),(4,5),(3,5),(3,4)\}$, para verificar se $P^{\prime}$ está no conjunto de matching da função $\operatorname{count}\left(p, P_{3}, f\right)$ são verificados os seguintes casos: $\left(i^{\prime}\right) P((0,1))=g \Rightarrow f(4,5) \geq f(4,4),\left(i i^{\prime}\right)$ $P((-1,1))=g \Rightarrow f(3,5) \geq f(4,4)$ e $\left(i i i^{\prime}\right) P((-1,0))=L \Rightarrow f(3,4)<f(4,4)$, porém, é verificado que o caso $\left(i i^{\prime}\right)$ não é verdadeiro, portanto, $P^{\prime} \notin \mathcal{M}_{P_{3}, f}(p)$ e é dito que $P^{\prime}$ não é contado por $\operatorname{count}\left(p, P_{3}, f\right)$.

A função count deve ser utilizada junto a uma max-tree ou min-tree com seus devidos padrões para a contagem de quads de todos os nós da árvore seja efetuado de maneira incremental. Porém semelhantemente ao trabalho de Climent e Oliveira [CO14] apenas a utilização dos padrões dos conjuntos em $\mathbb{P}$ e a soma das contagens dos nós filhos com a contagem nó pai não é suficiente, porque um quad contado no nó filho pode ter se transformado em outro quad em seu nó pai, quando isso ocorre é dito que houve uma transformação de padrão e a contagem do mesmo deve ser ajustada. A Fig. 3.4 exibe um exemplo de transformação. 

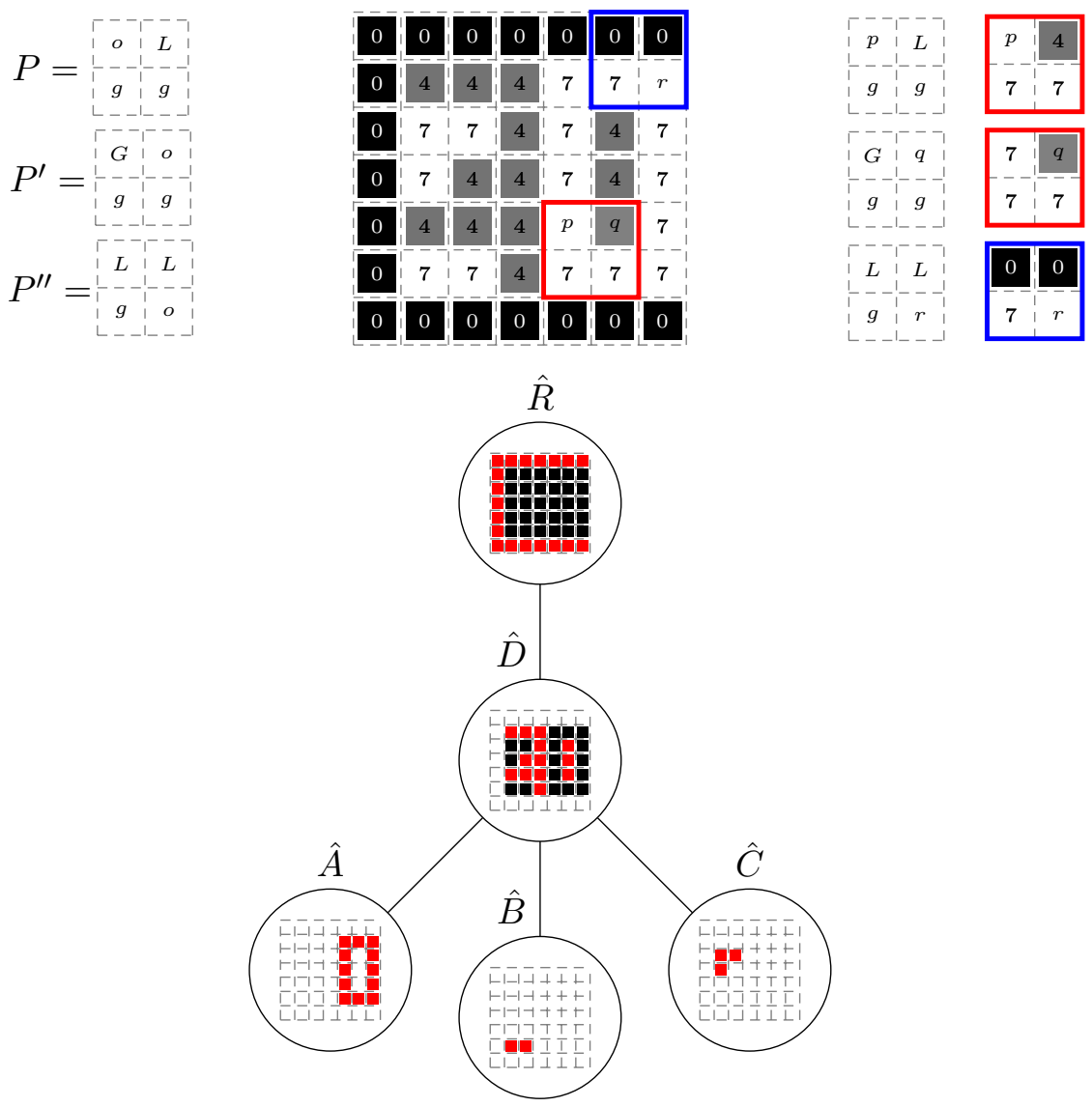

Fig. 3.4: Exemplo de transformação de padrões na contagem de quads. O padrão $P$ conta um quad do tipo $Q_{3}$ no nó $\hat{A}$ da árvore (quando o pixel $p$ é analisado), no nó $\hat{D}$ ao analisar o pixel q, o padrão $P^{\prime}$ é contado como um quad do tipo $Q_{4}$ para o mesmo conjunto de pixels (marcado como um retângulo vermelho) em que o padrão $P$ que representa um quad do tipo $Q_{3}$ havia sido contado, causando assim uma transformação de padrões. O padrão $P^{\prime \prime}$ que representada um quad do tipo $Q_{2}$ é contado no nó $\hat{A}$ e esse padrão/quad continua existindo no nó $\hat{D}$, neste caso não houve transformação de padrões.

Na Fig. 3.4, quando o nó $\hat{A}$ está sendo analisado o pixel $p \in \hat{A}=A$, o padrão $P \in P_{3}$ é contado, como o pixel $q \notin A=\hat{A}$ ele nunca é analisado para o nó $A$. O padrão $P \in P_{3}$ é contado porque existe um quad em $Q_{3}$ que é contado para vizinhança $D_{P}$ na imagem binária do nó $A$. Quando o nó $\hat{D}$ é analisado o padrão $P \in P_{3}$ que representa um quad do tipo $Q_{3}$ da imagem binária do nó $A$ já foi contado para o conjunto $D_{P}+p$ (isso pode acontecer em um método incremental que analisa o nó $\hat{A}$ antes do nó $\hat{D}$ ), porém esse quad não existe mais no nó $D$, porque o pixel de valor 0 necessário para esse quad se transformou em um pixel de valor 1 na imagem binária do nó $D$, isso é demonstrado pelo padrão $P^{\prime} \in P_{4}$ que é contado para o conjunto $D_{P^{\prime}}+q=D_{P}+p$ que representa um quad do tipo $Q_{4}$ contado nesse conjunto de pixels no nó $D$. Quando isso ocorre é dito que o quad do tipo $Q_{3}$ do nó $A$ se transformou em um quad do tipo $Q_{4}$ no nó $D$ e isso é denotado por $\left(Q_{3}, A\right) \Rightarrow\left(Q_{4}, D\right)$, ou simplesmente por $Q_{3} \Rightarrow Q_{4}$ quando a informação de nós não é necessária. Por outro lado o padrão $P^{\prime \prime} \in P_{2}$ é contado quando o pixel $r \in A \subset D$ do $\hat{A}$ é analisado, porque existe um quad do tipo $Q_{2}$ no conjunto $D_{P^{\prime \prime}}+r$ da imagem binária do nó $A$ e o mesmo quad continua existindo no nó $D$, portanto, não é necessário analisar o pixel $r$ novamente, ou seja, basta juntar esse quad(padrão) aos quads (padrões) contados no nó $D$.

São propostos os chamados padrões de transformação para identificar e contar os quads que foram transformados na transição entre nó filho e nó pai. Esses padrões de transformação são exibidos abaixo: 


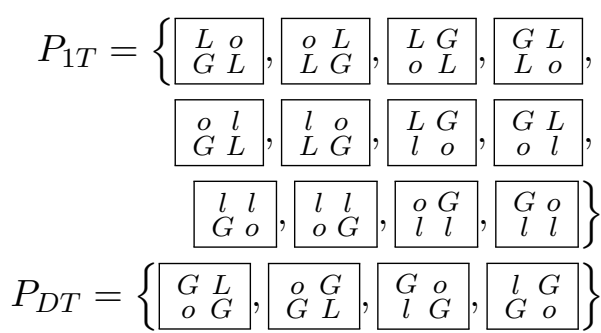

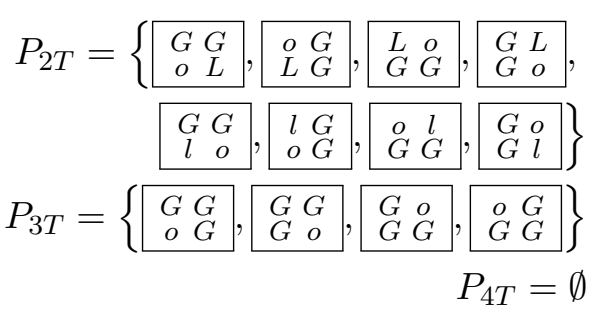

Os padrões de transformação apresentados são organizados em conjuntos que correspondem as possíveis transformações que os padrões/quads podem sofrer, ou seja, os padrões de $P_{1 T}$ contam transformações que ocorrem em quads do conjunto $Q_{1}\left(Q_{1} \Rightarrow Q_{i}\right.$, para $\left.i \in\{2,3,4, D\}\right)$. Os padrões de $P_{2 T}$ contam as transformações que ocorrem nos quads de $Q_{2}\left(Q_{2} \Rightarrow Q_{i}\right.$, para $\left.i \in\{3,4\}\right)$, os padrões de $P_{3 T}$ contam as transformações que ocorrem nos quads de $Q_{3}\left(Q_{3} \Rightarrow Q_{4}\right)$ e os padrões de $P_{D T}$ contam as transformações que ocorrem nos quads de $Q_{D}\left(Q_{D} \Rightarrow Q_{i}\right.$, para $\left.i \in\{3,4\}\right)$. Cada quad só pode se transformar em outro quad, se dentro de seu domínio transladado tenha pelo menos um pixel de valor 0 na imagem binária de um nó e esse(s) pixel(s) tenha $(\mathrm{m})$ seu valor transformado em 1 na imagem binária do nó pai desse nó. Portanto, quando existe a transformação $Q_{i} \Rightarrow Q_{j}$, então $j>i$ ou $i=D$ e $j \in\{3,4\}$. Dada essa relação entre os tipos de quads na transformação, é concluído que não existe transformação de um quad do tipo $Q_{4}$ e por convenção para qualquer pixel $p \in \mathcal{D}$ e imagem $f \in \mathcal{F}$, a função $\operatorname{count}\left(p, P_{4 T}, f\right)=0$.

A partir da definição desses padrões de transformação, o conjunto $\mathbb{P}$ é redefinido para $\mathbb{P}=$ $\left\{P_{1}, P_{2}, P_{3}, P_{4}, P_{D}, P_{1 T}, P_{2 T}, P_{3 T}, P_{4 T}, P_{D T}\right\}$. Quando se deseja denotar o conjunto de padrões de transformação de um conjunto $P_{i}$, é utilizado $P_{i T}$.

Os padrões de transformação são utilizados para remover as transformações de quads e assim definir o atributo de contagem de padrões para um dado nó $A$ da árvore de componentes e um índice $i \in\{1,2,3,4, D\}$ da seguinte maneira:

$$
\kappa_{P_{i}}(A)=\sum_{p \in A} \operatorname{count}\left(p, P_{i}, f\right)-\operatorname{count}\left(p, P_{i T}, f\right) .
$$

Um exemplo da utilização de um padrão de transformação pode ser observado na Fig. 3.4, onde mesmo contando o padrão $P \in P_{3}$ para o nó $D$ (através da contagem do nó $A$ que é nó filho do nó $D$ ) é identificado pelo padrão $\begin{array}{cc}G & o \\ G & G\end{array} \in P_{3 T}$ que o quad representado por esse padrão foi transformado, portanto, o mesmo deve ser removido da contagem de padrões do conjunto $P_{3}$ do nó $D$.

É necessário notar que os padrões propostos contam todos os quads para qualquer árvore de componentes de adjacência 8-conectividade, porém, os padrões de $P_{D}$ representam quads não conexos em adjacência de 4-conectividade, o que pode ocasionar falha ao contá-lo em uma árvore de componentes de adjacência 4-conectividade. Conforme descrito na Sec. 3.1.1, a interpretação adotada é que quads do tipo $Q_{D}$ contam dois quads do tipo $Q_{1}$, porque, os pixels de objeto dos quads de $Q_{D}$ estão separados, introduzindo assim os quads do tipo $Q_{1}^{\prime}$. Dado o cenário de quads diferentes para adjacência de 4-conectividade, os padrões utilizados para contar os quads do tipo $Q_{1}^{\prime}$ e seus padrões de transformação $\left(P_{1}^{\prime}\right.$ e $\left.P_{1 T}^{\prime}\right)$ são apresentados abaixo:

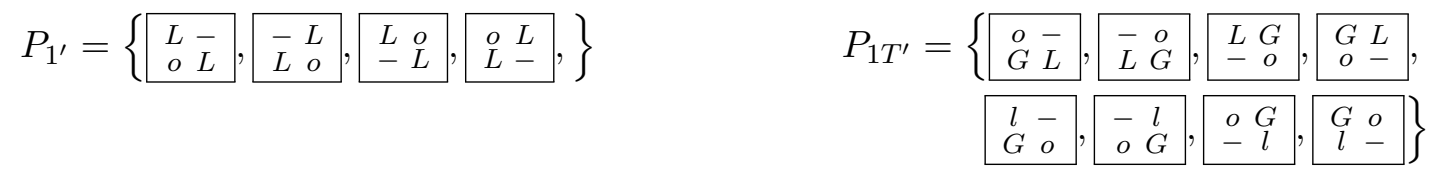

Portanto os conjuntos de padrões contados em árvores de componentes de adjacência 4-conectividade são: $P_{1^{\prime}}, P_{2}, P_{3}, P_{4}, P_{1 T^{\prime}}, P_{2 T}, P_{3 T}$. 


\subsection{Algoritmo incremental para contagem dos padrões propostos}

\subsubsection{Apresentação}

Nesta seção será apresentado o algoritmo que calcula a quantidade de quads para cada nó de uma árvore de componentes de maneira incremental, implementando os procedimentos e funções descritos na Sec. 2.2.2. De fato o algoritmo apresentado nesta seção é semelhante ao algoritmo proposto por Climent e Oliveira [CO14], porém está no formato do algoritmo incremental apresentado na Seção 2.2.2 e utiliza os padrões propostos na Sec. 3.1.2 que resulta na contagem de todos os quads propostos na Sec. 3.1.1 e consequentemente na contagem de todos os bit-quads propostos por Gray [Gra71]. Dessa forma, o algoritmo de Climent e Oliveira [CO14] pode ser adaptado para utilizar todos os padrões propostos ou o algoritmo descrito nessa seção pode ser adaptado para contar apenas os padrões propostos por Climent e Oliveira.

Para o método proposto nesta seção, os pixels de fora do domínio são considerados com intensidade $-\infty$ ou $+\infty$ dependendo da árvore de componentes utilizada. Quando a árvore de componentes de uma imagem $f: \mathcal{D} \rightarrow \mathbb{K}$ é construída a partir dos conjuntos de níveis superiores os pixels fora do domínio têm valor $-\infty$, porque dados o nó raiz $R=\mathcal{D}$, dois pixels vizinhos $p \in R$ e $q \notin R=\mathcal{D}$, a relação $f(p)>f(q)$ é mantida para qualquer valor finito de $f(p)$, ou seja, mesmo considerando os pixels fora do domínio do nó raiz $R$ de altitude $\lambda$ pode ser escrito como $R=\left\{p \in \mathbb{Z}^{2}: f(p) \geq \lambda\right\}$. Quando a árvore de componentes é construída utilizando os conjuntos de níveis inferiores o valor dos pixels fora do domínio tem valor $+\infty$, por motivo análogo a árvore de componentes dos conjuntos de níveis superiores.

O algoritmo proposto nesta seção considera: $(i)$ uma imagem em níveis de cinza $f: \mathcal{D} \rightarrow \mathbb{K}$, (ii) a árvore de componentes $T$ do conjunto de níveis superiores de $f$ utilizando adjacência 8-conectividade (note que os algoritmos são adaptáveis para a árvore de componentes de 4-conectividade), (iii) a árvore de componentes compacta e não redundante $\mathcal{T}$ de $T$ (max-tree), (iv) o conjunto de vértices $V_{T}$ da árvore $T,(v)$ o conjunto de vértices $V_{\mathcal{T}}$ da árvore $\mathcal{T},(v i)$ um nó $A \in V_{T}$ da árvore $T$ e (vii) seu nó associado na max-tree $\hat{A} \in V_{\mathcal{T}}$. O procedimento PRE-Process é responsável por contar os padrões para cada conjunto em todos os pixels do nó $\hat{A}$ na max-tree, conforme exibido no Alg. 9.

Alg 9: Função PRE-PROCESS do algoritmo de contagem de quads na max-tree.

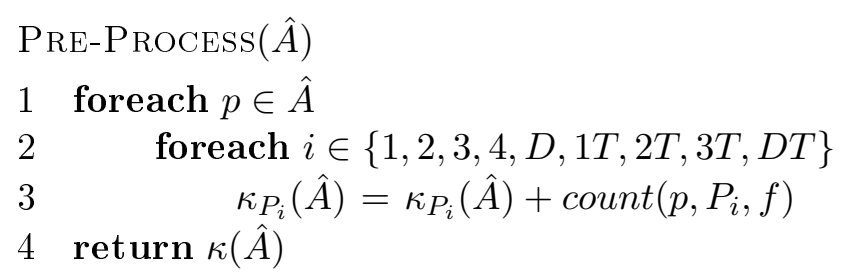

No Alg. 9, o primeiro laço percorre todos os pixels do nó $\hat{A}$ da max-tree e para cada pixel são percorridos todos os conjuntos de padrões $\left(P_{1}, P_{2}, P_{3}, P_{4}, P_{D}, P_{1 T}, P_{2 T}, P_{3 T}, P_{D T}\right)$, o conjunto $P_{4 T}=\emptyset$ e por isso não é avaliado. Em cada iteração, são acumuladas as quantidades de padrões encontrados em cada pixel em seus respectivos atributos: $\kappa_{P_{1}}(\hat{A}), \kappa_{P_{2}}(\hat{A}), \kappa_{P_{3}}(\hat{A}), \kappa_{P_{4}}(\hat{A}), \kappa_{P_{D}}(\hat{A})$, $\kappa_{P_{1 T}}(\hat{A}), \kappa_{P_{2 T}}(\hat{A}), \kappa_{P_{3 T}}(\hat{A})$ e $\kappa_{P_{D T}}(\hat{A})$ utilizando a função count. O procedimento MERGE que junta a contagem dos padrões dos nós filhos com o valor contado no nó pai é descrito no Alg. 10. 
Alg 10: Função Merge do algoritmo de contagem de quads na max-tree.

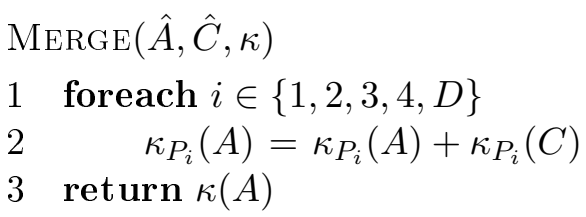

No Alg. 10, o número de padrões contados nos nós filhos do nó que está sendo analisado é acumulado, neste momento qualquer nó filho $\hat{C}$ do nó $\hat{A}$ já tem sua contagem finalizada pela chamada recursiva a Compute-AtTRIBute apresentada no algoritmo modelo Alg. 1 (veja Sec. 2.2.2), porém a contagem do nó $\hat{A}$ ainda não está finalizada por causa dos padrões que podem ter se transformado na transição de nó filho para nó pai, por isso é necessário a remoção (subtração) dos padrões de transformação que é realizada no procedimento Post-Process descrito no Alg. 11.

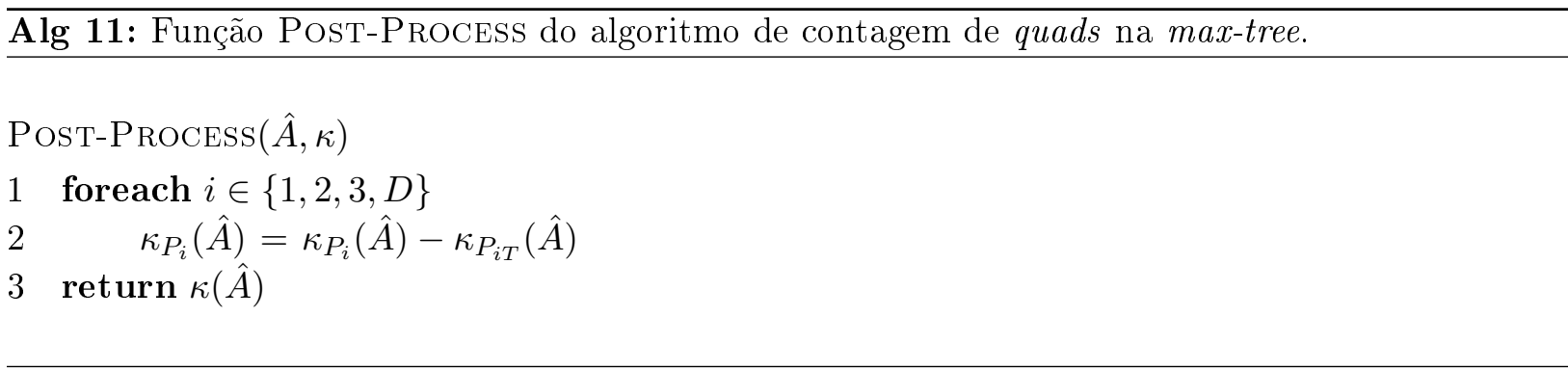

No Alg. 11, são removidos os padrões que foram transformados na transição de algum nó filho para o nó pai. Essas transformações são capturadas pelos padrões de transformação e o ajuste necessário é a subtração do total de padrões contados no nó (incluindo a dos nós filhos) com o total de padrões de transformação contados (as transformações ocorridas nos nós descendentes do nó analisado $\hat{A}$ são contadas quando o procedimento recursivo Compute-ATTRIBUTE é executado, por isso a operação de MERge já leva essas transformações em consideração e a operação Post-PROcEss não necessita utilizar esses valores explicitamente). A Fig. 3.5 exibe um exemplo da execução do algoritmo com todos os procedimentos descritos nos Algs. 9, 10 e 11. 


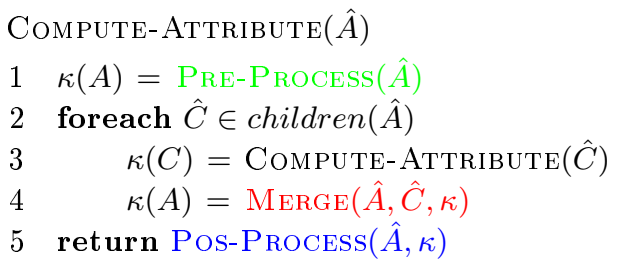

$1 \kappa(A)=\operatorname{PrE}-\operatorname{PrOCESS}(\hat{A})$

2 foreach $\hat{C} \in \operatorname{children}(\hat{A})$

5 return Pos-Process $(\hat{A}, \kappa)$

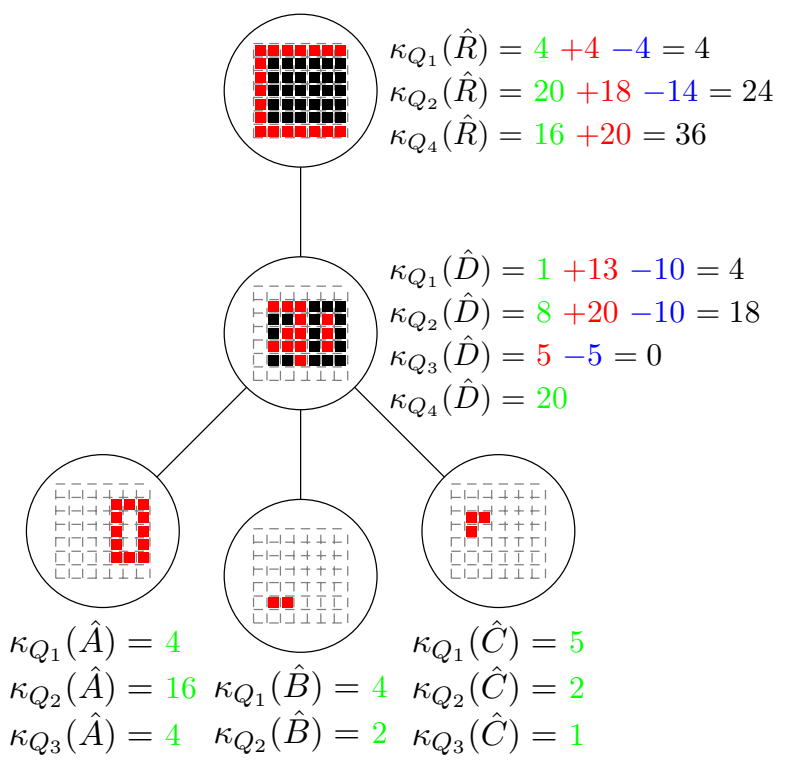

Fig. 3.5: Contagem de padrões utilizando o algoritmo incremental. Os números em verde indicam a contagem realizada pela função PRE-PROCESS, os números em vermelho indicam a contagens acumuladas dos padrões nos nós filhos realizadas pelo procedimento MERGE após todas as iteraçôes do laço do algoritmo, os números em azul indicam a remoção dos quads transformados realizada pelo procedimento POST-PROCESS. Os procedimentos que realizam operações com o valor 0 foram suprimidos.

A Fig. 3.5 exibe o funcionamento do algoritmo que conta todos os quads para todos os nós de uma árvore de componentes. O algoritmo inicia-se contando os padrões no nó raiz $\hat{R}$ pela chamada da função Pre-Process (número em verde), em seguida o algoritmo entra no laço dos filhos de $\hat{R}$ chamando a função Compute-ATTRIBUTE recursivamente para seu único filho $\hat{D}$. No processamento do nó $\hat{D}$, a contagem dos padrões é feita pela função PRE-PROCEss (número verde) e então são realizadas chamadas recursivas para os seus nós filhos $\hat{A}, \hat{B}, \hat{C}$. Os nós $\hat{A}, \hat{B}$ e $\hat{C}$ são nós folhas e por isso apenas a função PRE-Process tem efeito (número em verde), porque eles não têm nós filhos e consequentemente nenhuma iteração do laço é executada, e também porque nenhum padrão de transformação é contado (note que todos os padrões de transformação possuem um símbolo $G$ e os nós folhas são máximos locais, portanto, não existem padrões de transformação em nós folhas). Então o algoritmo volta a processar o nó $\hat{D}$ acumulando as contagens de padrões dos nós $\hat{A}, \hat{B}$ e $\hat{C}$ com a operação Merge (em vermelho), em seguida o algoritmo remove os quads que foram transformados na transição entre os nós filhos e o nó $\hat{D}$ chamando o procedimento Post-Process (número em azul) e finalizando a contagem de padrões do nó $\hat{D}$. Por último, o processamento do algoritmo volta ao nó $\hat{R}$ acumulando sua contagem a contagem no nó $\hat{D}$ pela chamada a MERGE (número em vermelho) e removendo as transformações de quads pela chamada a PosT-PROCEss (número em azul), finalizado assim a contagem de quads para todos os nós da árvore.

Em cada chamada o algoritmo proposto percorre todos os pixels de um nó compacto e não redundante e para cada pixel a função de contagem é chamada uma vez. Cada pixel da imagem está armazenado em apenas um nó da max-tree ou min-tree, portanto, a função de contagem é chamada apenas uma vez para cada pixel da imagem gastando tempo proporcional a $O(|\mathcal{D}|)$. Além disso, o algoritmo proposto executa as operações MERGE para cada aresta da árvore e a função Post-Process para cada nó. Cada uma dessas operações gasta tempo constante. Consequentemente, para uma árvore de componentes formada pelo conjunto $V_{T}$ de nós e $E_{T}$ de arestas(definido pela relação de parentesco) o custo do algoritmo é dado por

$$
O(|\mathcal{D}|)+O\left(\left|V_{T}\right|\right)+O\left(\left|E_{T}\right|\right) .
$$

Como o números de nós de uma árvore de componentes não é maior que o número de pixels da imagem que ela representa, e por ser tratar de uma árvore, vale a relação $\left|E_{T}\right|=\left|V_{T}\right|-1$. É 
concluido que o algoritmo gasta tempo linear no número de pixels $O(|\mathcal{D}|)$ em seu pior caso.

\subsubsection{Corretude}

A corretude do algoritmo se baseia no fato de que a contagem de quads de cada tipo (conjunto) na imagem binária do nó ser a mesma obtida pelo algoritmo proposto, isso pode ser resumido no seguinte teorema.

Teo 1. Seja $f: \mathcal{D} \rightarrow \mathbb{K}$ uma imagem em niveis de cinza, $T$ a árvore de componentes construída a partir dos conjuntos de niveis superiores de $f$ e uma relação de adjacência $\mathcal{A}\left(\mathcal{A}_{4}\right.$ ou $\left.\mathcal{A}_{8}\right)$ e $V_{T}$ os nós dessa árvore, então para qualquer nó $A \in V_{T}$ vale a seguinte equação:

$$
\kappa_{Q_{i}}(A)=\kappa_{P_{i}}(A),
$$

para qualquer $i \in\{1,2,3,4, D\}$ quando $T$ é uma árvore de componentes de adjacência 8-conectividade e $i \in\{1,2,3,4\}$ quando $T$ é uma árvore de componentes de adjacência de 4-conectividade.

A prova do Teo. 1 é construída analisando as translações dos domínios dos padrões propostos e verificando que para cada conjunto de pixels vizinhos formado por uma translação do domínio de um padrão contado tem um quad correspondente com o mesmo índice e vice-versa (considerando a remoção dos padrões de transformação). A translação do domínio de um padrão resulta em um conjunto de quatro pontos vizinhos do $\mathbb{Z}^{2}$, para representar esses pontos, é definido o conceito de janela de tamanho $2 \times 2$. Uma quadrupla $\left\{\left(x_{1}, y_{1}\right),\left(x_{2}, y_{2}\right),\left(x_{3}, y_{3}\right),\left(x_{4}, y_{4}\right)\right\}$ de pixels é uma janela de tamanho $2 \times 2$, se e somente se, $\left(x_{2}, y_{2}\right)=\left(x_{1}+1, y_{1}\right),\left(x_{3}, y_{3}\right)=\left(x_{1}, y_{1}+1\right)$ e $\left(x_{4}, y_{4}\right)=$ $\left(x_{1}+1, y_{1}+1\right)$. Os pixels de uma janela são nomeados como $t l=\left(x_{1}, y_{1}\right), t r=\left(x_{2}, y_{2}\right), b l=\left(x_{3}, y_{3}\right)$

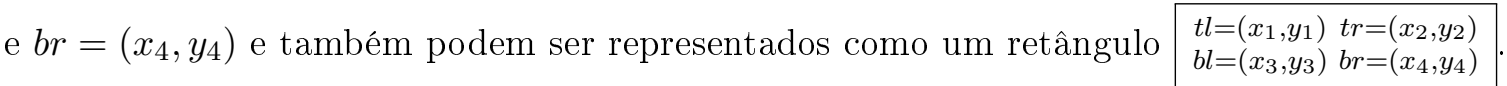

O conjunto de todas as possíveis janelas de tamanho $2 \times 2$ de $\mathbb{Z}^{2}$ é denotado por $\mathcal{W}$ e o conjunto de todas as janelas que tenham intersecção com um dado subconjunto de pixels $A \in \mathbb{Z}^{2}$ é denotado por $\mathcal{W}(A)=\{W \in \mathcal{W}:(W \cap A) \neq \emptyset\}$.

Dados o conceito de janela de tamanho $2 \times 2$ e um nó $A$ de uma árvore de componentes, é possível identificar os quads de um conjunto em $\mathcal{Q}$ que estão contidos em uma janela $W \in \mathcal{W}(A)$, ou seja, dada uma tripla $\left(W, B_{i}, A\right) \in \mathcal{W} \times \mathcal{B} \times \mathcal{P}(\mathcal{D})$, o conjunto de matching de quads contidos em uma janela é definido como:

$$
M W_{Q_{i}, A}(W)=\left\{Q \in Q_{i}: p \in(W \cap A), Q \in M_{Q_{i}, A}(p), W=D_{Q}+p\right\} .
$$

A partir da definição do conjunto de matching em uma janela, a função de contagem de quads em uma janela count $B W: W \times \mathcal{Q} \times \mathcal{P}(\mathcal{D}) \rightarrow \mathbb{Z}$ é definida para uma tripla $\left(W, Q_{i}, A\right) \in \mathcal{W} \times \mathcal{Q} \times \mathcal{P}(\mathcal{D})$ da seguinte maneira:

$$
\text { count } B W\left(W, Q_{i}, A\right)=\left|M W_{Q_{i}, A}(W)\right| .
$$

Quando um quad $Q \in M W_{Q_{i}, A}(W)$, é dito que o quad foi contando por count $B W\left(W, Q_{i}, A\right)$, caso contrário, é dito que o quad não foi contado por count $B W\left(W, Q_{i}, A\right)$. A Fig. 3.6 exibe exemplos de janelas em uma imagem binária.
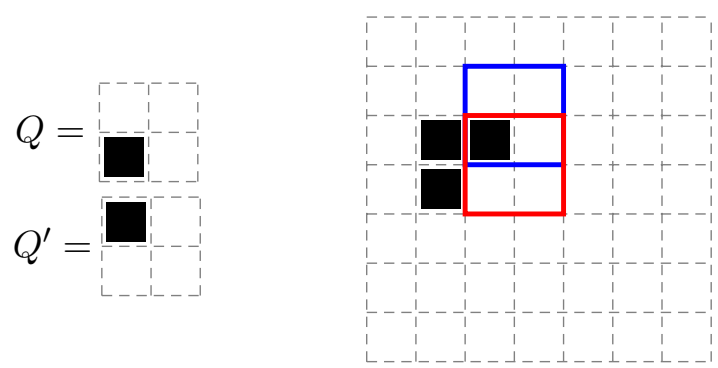

Fig. 3.6: Exemplos de duas janelas formadas por translaçôes dos dominios dos quads $Q$ e $Q^{\prime}$. A janela $W$ é formada por $D_{Q}+p$ (em azul) e a janela $W^{\prime}$ é formada por $D_{Q^{\prime}}+p$ (em vermelho). 
Nos exemplos da Fig. 3.6, são ilustradas duas janelas: $W=D_{Q}+p$ (em azul) e $W^{\prime}=$ $D_{Q^{\prime}}+p$ (em vermelho). Observando a Fig. 3.6, é possível verificar que o quad $Q$ será contado por count $B W\left(W, Q_{1}, A\right)$ (onde $A$ é a imagem binária representada na figura), porque $Q \in M_{A, Q_{1}}(p)$ e $W=\left(D_{Q}+p\right)$, porém, $Q^{\prime}$ não será contado, porque, $W \neq D_{Q^{\prime}}+p$. O quad $Q^{\prime}$ será contado por count $B W\left(W^{\prime}, Q_{1}, A\right)$, porque, $Q^{\prime} \in M_{A, Q_{i}}(p)$ e $W^{\prime}=D_{Q^{\prime}}+p$, porém, $Q$ não será contado, porque, $W^{\prime} \neq D_{Q}+p$. Analogamente a função count $B$ e o atributo $\kappa_{Q_{i}}$, é definido o atributo $\kappa W_{Q_{i}}(A)$ para uma imagem binária $A$ da seguinte maneira:

$$
\kappa W_{Q_{i}}(A)=\sum_{W \in \mathcal{W}(A)} \operatorname{count} B W\left(W, Q_{i}, A\right) .
$$

Similarmente é possível identificar os padrões propostos no algoritmo incremental contidos em uma janela de tamanho $2 \times 2$ de um nó $A$ de uma árvore de componentes. O conjunto de matching de padrões dentro de uma janela é definido pela quadrupla $\left(W, P_{i}, f, A\right) \in \mathcal{W} \times \mathbb{P} \times \mathcal{F} \times \mathcal{P}(\mathcal{D})$ da seguinte forma:

$$
\mathcal{M} W_{f, P_{i}, A}(W)=\left\{P \in P_{i}: p \in(W \cap A), P \in \mathcal{M}_{f, P_{i}}(p), W=\left(D_{P}+p\right)\right\}
$$

A função de contagem de padrões dentro de uma janela countW $: \mathcal{W} \times \mathbb{P} \times \mathcal{F} \times \mathcal{P}(\mathcal{D}) \rightarrow \mathbb{Z}$ é definida a partir do conjunto de matching de padrões dentro de uma janela para a quadrupla $\left(W, P_{i}, f, A\right) \in \mathcal{W} \times \mathbb{P} \times \mathcal{F} \times \mathcal{P}(\mathcal{D})$ da seguinte maneira:

$$
\operatorname{count} W\left(W, P_{i}, f, A\right)=\left|\mathcal{M} W_{f, P_{i}, A}(W)\right| .
$$

É dito que um padrão $P$ é contado por count $W\left(W, P_{i}, f, A\right)$, se $P \in \mathcal{M} W_{f, P_{i}, A}(W)$ e o padrão $P$ não é contado, caso $P \notin \mathcal{M} W_{f, P_{i}, A}(W)$. Exemplos de janelas utilizadas na função count $W$ são exibidos na Fig. 3.7.

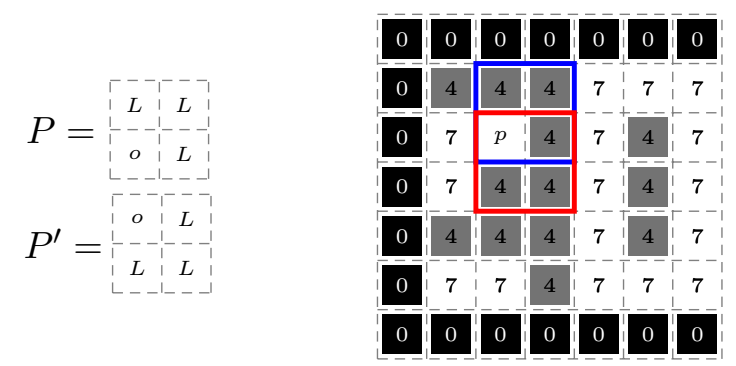

Fig. 3.7: Janelas em uma imagem em nivel de cinza definidas por translação dos domínios dos padrões $P$ (em azul) e $P^{\prime}$ (em vermelho).

É possível verificar na Fig. 3.7 que o padrão $P$ é contado pela função count $W\left(W, P_{i}, f, A\right)$, para $W=\left(D_{P}+p\right)$ e $A=\mathcal{S C}(\mathcal{T}, p)$ e $P^{\prime}$ não é contado por essa função. O padrão $P^{\prime}$ é contado pela função count $W\left(W^{\prime}, P_{i}, f, A\right)$, para $W^{\prime}=D_{P^{\prime}}+p$ e o padrão $P$ não é contado para essa função. Analogamente a contagem de quads por janelas, o atributo de contagem de padrões na janela para um nó $A$ de uma árvore de componentes é definido como:

$$
\kappa W_{P_{i}}(A)=\sum_{W \in \mathcal{W}(A)} \operatorname{count} W\left(W, P_{i}, f, A\right)-\operatorname{count} W\left(W, P_{i T}, f, A\right) .
$$

Para provar o Teo 1 são considerados: $(i)$ uma imagem em nível de cinza $f: \mathcal{D} \rightarrow \mathbb{K}$, (ii) uma árvore de componentes $T$ construída a partir dos conjuntos de níveis superiores de $f$ e a relação de adjacência $\mathcal{A}\left(\mathcal{A}_{4}\right.$ ou $\left.\mathcal{A}_{8}\right)$, $($ iii $) V_{T}$ o conjunto de nós de $T,(i v) A \in V_{T}$ um nó da árvore $T,(v)$ $Q_{i} \in \mathcal{Q}$ um conjunto de quads e (vi) $P_{i} \in \mathbb{P}$ um conjunto de padrões.

A prova do Teo. 1 consiste em vários passos que formam um conjunto de proposições. A visão geral da prova é apresentada abaixo: 
1. Provar que para qualquer $W \in \mathcal{W}(A)$, count $B W\left(W, Q_{i}, A\right)=1$ ou count $B W\left(W, Q_{i}, A\right)=0$.

2. Provar que $\kappa_{Q_{i}}(A)=\kappa W_{Q_{i}}(A)$.

3. Provar que para qualquer janela $W \in \mathcal{W}(A)$, count $W\left(W, P_{i}, f, A\right)=1$ ou $\operatorname{count} W\left(W, P_{i}, f, A\right)=$ 0 .

4. Provar que para qualquer janela $W \in \mathcal{W}(A)$, count $W\left(W, P_{i T}, f, A\right)=1$ ou count $W\left(W, P_{i T}, f, A\right)=$ 0 .

5. Provar que para qualquer janela $W \in \mathcal{W}(A)$, count $W\left(W, P_{i}, f, A\right)-\operatorname{count} W\left(W, P_{i T}, f, A\right)=1$ ou count $W\left(W, P_{i}, f, A\right)-\operatorname{count} W\left(W, P_{i T}, f, A\right)=0$.

6. Provar que $\kappa_{P_{i}}(A)=\kappa W_{P_{i}}(A)$.

7. Provar que count $W\left(W, P_{i}, f, A\right)-\operatorname{count} W\left(W, P_{i T}, f, A\right)=\operatorname{count} B W\left(W, Q_{i}, A\right)$.

8. Provar que $\kappa W_{Q_{i}}(A)=\kappa W_{P_{i}}(A)$.

O teorema exige uma prova para todos os valores possíveis de $i(1,2,3,4, D)$. A estratégia adotada para a prova apresentada no texto é demonstrar que o teorema é verdadeiro para cada valor de $i$ diferente, porém, como a prova é muita extensa e também análoga ou semelhante para qualquer valor de $i$, no texto apenas a prova para $i=3$ é exibida:

Prop 3.1. (passo 1.) Seja $W$ uma janela de tamanho $2 \times 2$ do nó $A$, então count $B W\left(W, Q_{i}, A\right)=1$ ou count $B W\left(W, Q_{i}, A\right)=0$.

Demonstração. A prova é feita para o caso $i=3$. Outros casos podem ser provados de forma análoga.

Se $|W \cap A| \neq 3$, então count $B W\left(W, Q_{3}, A\right)=0$, por definição de $Q_{3}$, caso contrario $(|W \cap A|=3)$, é necessário avaliar as possíveis combinações de valores dentro de $W \cap A$, por exemplo:

- Se $W \cap A=\begin{array}{ll}1 & 1 \\ 0 & 1\end{array}$, então existe $p \in W$, tal que $\left.\begin{array}{ll}1 & 1 \\ 0 & o\end{array}\right] \in M_{A, Q_{3}}(p)$ e $\left(D_{P}+p\right)=W$, também é observável que os quads $\begin{array}{ll}0 & 0 \\ 1 & 1\end{array}, \begin{array}{ll}1 & 1 \\ o & 0\end{array}, \begin{array}{ll}o & 0 \\ 1 & 1\end{array}$ não estão em $M_{A, Q_{3}}(p)$ para qualquer $p \in(W \cap A)$, porque o pixel bl $\notin A$, portanto, count $B W\left(W, Q_{3}, A\right)=1$.

Os outros três casos são análogos ao caso apresentado acima, para verificar os outros casos é necessário verificar que a posição do pixel com valor 0 no quad analisado sempre terá valor 1 nos outros quads (não analisados). Logo é concluído que count $B W\left(W, Q_{3}, A\right)=1$ ou count $B W\left(W, Q_{3}, A\right)=0$.

Prop 3.2. (passo 2.)

$$
\kappa_{Q_{3}}(A)=\kappa W_{Q_{3}}(A)
$$

Demonstração. A prova é feita em dois passos:

- $\kappa_{Q_{i}}(A) \geq \kappa W_{Q_{i}}(A)$

Dada uma janela $W$ de tamanho $2 \times 2$, se não existe um quad $Q \in M W_{Q_{i}, A}(W)$, então $\kappa W_{Q_{i}}(A)=0$ e $\kappa_{Q_{i}}(A) \geq \kappa W_{Q_{i}}(A)$ é trivialmente verdade. Caso contrário, $\exists Q \in Q_{i}$, tal que $Q \in M W_{Q_{i}, A}(W)$, então por definição de $M Q_{Q_{i}, A}(W)$, existe $p \in(A \cap W) \subseteq A$, tal que $Q \in M_{Q_{i}, A}(p)$ isso implica que quando $Q$ é contado pela função count $B W$ ele também é contado pela função count $B$, logo é concluído que $\kappa_{Q_{i}}(A) \geq \kappa W_{Q_{i}}(A)$.

- $\kappa_{Q_{i}}(A) \leq \kappa W_{Q_{i}}(A)$

Dado um pixel $p \in A$, se não existe um quad $Q \in M_{Q_{i}, A}(p)$ então $\kappa_{Q_{i}}(A)=0$ e $\kappa_{Q_{i}}(A) \leq$ $\kappa_{Q_{i}}(A)$ é trivialmente verdade. Caso contrário, $\exists Q \in Q_{i}$, tal que $Q \in M_{Q_{i}, A}(p)$ então também existe $W=\left(D_{P}+p\right)$, e obviamente $W \in \mathcal{W}(A), \log 0 \quad Q \in M W_{Q_{i}, A}(W)$ e $Q$ é contado por count $B W\left(W, Q_{i}, A\right)$, portanto, é concluído que $\kappa_{Q_{i}}(A) \leq \kappa W_{Q_{i}}(A)$. 
Mostrando os dois casos acima, é possível verificar $\kappa_{Q_{i}}(A)=\kappa W_{Q_{i}}(A)$.

Prop 3.3. (passo 3.) Seja $W$ uma janela de tamanho $2 \times 2$ do nó $A$, então count $W\left(W, P_{i}, f, A\right)=1$ ou count $W\left(W, P_{i}, f, A\right)=0$.

Demonstração. A prova é feita para o caso $i=3$. Outros casos podem ser provados de forma análoga.

Se não existir algum $P \in \mathcal{M} W_{P_{3}, f, A}(W)$ então nada a provar, caso contrário existe um padrão $P \in \mathcal{M} W_{P_{3}, f, A}(W)$, suponha por contradição que exista um outro padrão $P^{\prime} \neq P$, tal que $P^{\prime} \in$ $\mathcal{M} W_{P_{3}, f, A}(W)$, então são analisadas todas as possibilidades que $P, P^{\prime} \in P_{3}$ possam assumir, por exemplo:

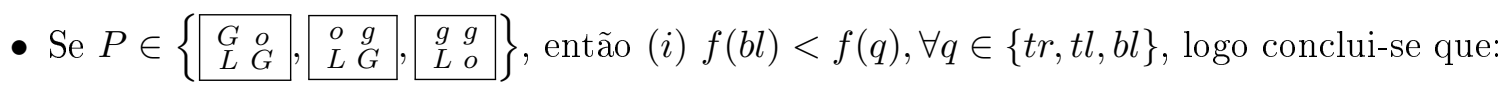

- $\left.P^{\prime} \notin\left\{\begin{array}{ll}o & G \\ G & L\end{array}, \begin{array}{ll}o & L \\ g & g\end{array}\right]\right\}$, caso contrário, $f(b l) \geq f(t l)$ que contradiz $(i)$.

$\left.-P^{\prime} \notin\left\{\begin{array}{ll}g & o \\ G & L\end{array}, \begin{array}{ll}L & o \\ g & g\end{array}\right]\right\}$, caso contrário, $f(b l) \geq f(t r)$ que contradiz $(i)$.

$-P^{\prime} \notin\left\{\begin{array}{ll}G & L \\ g & o\end{array},, \begin{array}{ll}L & G \\ G & o\end{array}\right\}$, caso contrário, $f(b l) \geq f(b r)$ que contradiz $(i)$.

$-P^{\prime} \neq \begin{array}{ll}g & g \\ o & L\end{array}$, caso contrário, $f(b l)>f(b r), P^{\prime} \neq \begin{array}{cc}G & L \\ o & G\end{array}$, caso contrário, $f(b l)>f(t r)$ e $P^{\prime} \neq \begin{gathered}L \\ o g\end{gathered}$, caso contrário $f(b l)>f(t l)$, em todos esses casos ocorrem uma contradição $\operatorname{com}(i)$.

Agora analisando se $\left.P^{\prime}, P \in\left\{\begin{array}{ll}G & o \\ L & G\end{array},, \begin{array}{ll}o & g \\ L & G\end{array},, \begin{array}{ll}g & g \\ L & o\end{array}\right]\right\}$, é concluído que:

$-P=\begin{array}{ll}G & o \\ L & G\end{array} \Rightarrow f(t l)>f(t r)$ e $f(b r)>f(t r), \log 0 P^{\prime} \neq \begin{array}{ll}o & g \\ L & G\end{array}$, caso contrário, $f(t l) \leq$ $f(t r)$ e $\left.P^{\prime} \neq \begin{array}{l}g \\ g \\ L\end{array}\right]$, caso contrário, $f(b r) \leq f(t r)$, portanto, para esse caso $P^{\prime}$ não existe.

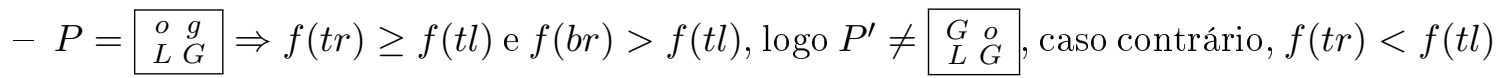
e $P^{\prime} \neq \begin{gathered}g \\ L\end{gathered}$

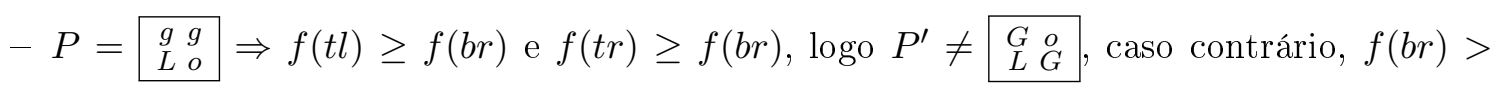
$f(t r)$ e $P^{\prime} \neq \begin{array}{ll}o & g \\ L & G\end{array}$, caso contrário, $f(b r)>f(t l)$, portanto, neste caso $P^{\prime}$ não existe.

Os casos em que $P \in\left\{\begin{array}{ll}o & G \\ G & L\end{array},, \begin{array}{ll}g & o \\ G & L\end{array},, \begin{array}{ll}g & g \\ o & L\end{array}\right], P \in\left\{\begin{array}{ll}G & L \\ o & G\end{array},, \begin{array}{ll}G & L \\ g & o\end{array},, \begin{array}{ll}o & L \\ g & g\end{array}\right\}$ e $P \in\left\{\begin{array}{lll}L & G \\ G & o\end{array},, \begin{array}{ll}L & G \\ o & g\end{array},, \begin{array}{ll}L & o \\ g & g\end{array}\right\}$ são análogos. A partir da análise desses casos é concluído que $P^{\prime}$ não existe, portanto, a função de contagem nas janelas só pode resultar em um ou zero.

Prop 3.4. (passo 4.) Seja $W$ uma janela de tamanho $2 \times 2$ do nó $A$, então count $W\left(W, P_{i T}, f, A\right)=$ 1 ou count $W\left(W, P_{i T}, f, A\right)=0$.

Demonstração. A prova é feita para o caso $i=3$. Outros casos podem ser provados de forma análoga.

Se count $W\left(W, P_{3 T}, f, A\right)=0$, então nada a provar. Caso contrário, existe pelo menos um padrão $P \in P_{3 T}$, tal que $P \in \mathcal{M} W_{P_{3 T}, f, A}(W)$, e um pixel $p \in(W \cap A)$ tal que $(i) f(p)<f(r), \forall r \in$ $((W \cap A)-p)$, agora suponha por contradição que também exista um padrão $P^{\prime} \in P_{3 T}\left(P \neq P^{\prime}\right)$ e $P^{\prime} \in \mathcal{M} W_{P_{3 T}, f, A}(W)$, isso implica que existe um pixel $q \in(W \cap A)$, tal que $f(q)<f(r), \forall r \in$ $((W \cap A)-q)$, mas isso contradiz $(i)$, por inspeção $\left(Q_{3 T}=\left\{\begin{array}{cc}G & G \\ 0 & G\end{array},, \begin{array}{cc}G & G \\ G & o\end{array},, \begin{array}{ll}G & o \\ G & G\end{array},, \begin{array}{cc}o & G \\ G & G\end{array}\right]\right)$ é verificado que isso é verdade para qualquer par de padrões $P$ e $P^{\prime}$, portanto, count $W\left(W, P_{3 T}, f, A\right)=1$ ou count $W\left(W, P_{3 T}, f, A\right)=0$. 
Prop 3.5. (passo 5.) Seja $W$ uma janela de tamanho $2 \times 2$ de $A$, então count $W\left(W, P_{i}, f, A\right)$

$-\operatorname{count} W\left(W, P_{i T}, f, A\right)=0$ ou count $W\left(W, P_{i}, f, A\right)-\operatorname{count} W\left(W, P_{i T}, f, A\right)=1$

Demonstração. A prova é feita para o caso $i=3$. Outros casos podem ser provados de forma análoga.

Pela Prop. 3.3, count $W\left(W, P_{3}, f, A\right)=1$ ou count $W\left(W, P_{3}, f, A\right)=0$ e pela Prop. 3.4, $\operatorname{count} W\left(W, P_{3 T}, f, A\right)=1$ ou count $W\left(W, P_{3 T}, f, A\right)=0$, portanto, se count $W\left(W, P_{3}, f, A\right)=1$ ou count $W\left(W, P_{3 T}, f, A\right)=0$, então a proposição é trivialmente verdadeira. A única combinação não válida para a proposição é count $W\left(W, P_{3 T}, f, A\right)=1$ e count $W\left(W, P_{3}, f, A\right)=0$, portanto, a prova é dada analisando cada possível padrão $P \in P_{3 T}$ que pode gerar count $W\left(W, P_{3 T}, f, A\right)=1$, um exemplo é exibido abaixo:

- Se $\left.P=\begin{array}{c}G \\ o \\ o\end{array}\right]$, então $f(b l)<f(q), \forall q \in\{t l, t r, b r\}$, analisando o valor mínimo $\lambda=\min \{f(q)$ : $q \in\{t l, t r, b r\}\}$ é possível concluir que:

- Se $f(b r)=\lambda$, então trivialmente $\left.\begin{array}{l}g \\ L \\ L\end{array}\right] \in \mathcal{M} W_{P_{3}, f, A}(W)$ e count $W\left(W, P_{3}, f, A\right)=1$ (prop. 3.3).

- Se $f(t l)=\lambda$, então pode ocorrer um dos casos abaixo:

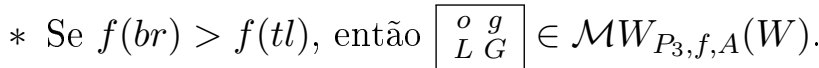

* Se $f(t l)=f(b r)$, então $\begin{array}{rl}g & g \\ L & o\end{array} \in \mathcal{M} W_{P_{3}, f, A}(W)$.

Em ambos os casos count $W\left(W, P_{3}, f, A\right)=1$ (prop. 3.3).

- Se $f(t r)=\lambda$, então pode ocorrer um dos casos abaixo:

* Se $f(t l)>f(t r)$ e $f(b r)>f(t r)$, então $\begin{array}{lll}G & \stackrel{o}{G}\end{array} \in \mathcal{M} W_{P_{3}, f, A}(W)$.

* Se $f(t r)=f(t l)$ e $f(b r)>f(t r)$, então $\begin{array}{ll}o & g \\ L & G\end{array} \in \mathcal{M} W_{P_{3}, f, A}(W)$.

* Se $f(t l)=f(b r)=f(t r)$, então $\left.\begin{array}{ll}g & g \\ L & o\end{array}\right] \in \mathcal{M}_{P_{3}, f, A}(W)$.

Em todos os casos count $W\left(W, P_{3}, f, A\right)=1$ (prop. 3.3).

Os casos em que $P=\underset{G}{G} \underset{G}{G}, \quad, \begin{array}{cc}G & o \\ G & G\end{array}$ ou $\begin{array}{cc}o & G \\ G & G\end{array}$ são análogos. A partir da análise de todos os casos, é possível concluir que count $W\left(W, P_{3}, f, A\right)=1$, portanto, a proposição é verdadeira.

Prop 3.6. (passo 6.) $\kappa_{P_{i}}(A)=\kappa W_{P_{i}}(A)$.

Demonstração. Seja $\Phi_{P_{i}}(A)=\sum_{p \in A} \operatorname{count}\left(p, P_{i}, f\right)$ e $\Phi W_{P_{i}}(A)=\sum_{W \in \mathcal{W}(A)} \operatorname{count} W\left(W, P_{i}, f, A\right)$, então como passo intermediário para a prova da proposição é demonstrado que $\Phi_{P_{i}}(A)=\Phi W_{P_{i}}(A)$ (para qualquer $i \in\{1,2,3,4, D, 1 T, 2 T, 3 T, D T\}$ ), analisando as seguintes relações:

- $\Phi_{P_{i}}(A) \geq \Phi W_{Q_{i}}(A)$.

Dada uma janela $W \in \mathcal{W}(A)$, se não existir um padrão $P \in \mathcal{M} W_{P_{i}, f, A}(W)$ então $\Phi W_{P_{i}}(A)=0$ e $\Phi_{P_{i}}(A) \geq \Phi W_{P_{i}}(A)$ é trivialmente verdade, caso contrário, existe um padrão $P \in \mathcal{M} W_{P_{i}, f, A}(W)$ então por definição existe um pixel $p \in(W \cap A)$, tal que $P \in \mathcal{M}_{P_{i}, f}(p)$, ou seja, quando o padrão $P$ é contado por uma função count $W$ ele também é contado por uma função count, portanto, $\Phi_{P_{i}}(A) \geq \Phi W_{P_{i}}(A)$.

- $\Phi_{P_{i}}(A) \leq \Phi W_{P_{i}}(A)$.

Dado um pixel $p \in A$, se não existir um padrão $P \in \mathcal{M}_{P_{i}, f}(p)$ então $\Phi_{P_{i}}(A)=0$ e $\Phi_{P_{i}}(A) \leq$ $\Phi W_{Q_{i}}(A)$ é trivialmente verdade, caso contrário, existe um padrão $P \in \mathcal{M}_{P_{i}, f}(p)$, então por definição existe uma janela $W=D_{P}+p$ de tamanho $2 \times 2$, tal que $p \in(W \cap A)$ e $P \in \mathcal{M} W_{P_{i}, f, A}(W)$, ou seja, quando o padrão $P$ é contado por uma função count, ele também é contado por uma função count $W$, logo é concluído que $\Phi_{P_{i}}(A) \leq \Phi W_{P_{i}}(A)$. 
Dadas as decomposições: $\kappa_{P_{i}}(A)=\Phi_{P_{i}}(A)-\Phi_{P_{i T}}(A)$ e $\kappa W_{P_{i}}(A)=\Phi W_{P_{i}}(A)-\Phi W_{P_{i T}}(A)$, pela demonstração acima é conhecido que: $\Phi_{P_{i}}(A)=\Phi W_{P_{i}}(A)$ e $\Phi_{P_{i T}}(A)=\Phi W_{P_{i T}}(A)$, portanto, é concluído que $\kappa_{P_{i}}(A)=\kappa W_{P_{i}}(A)$.

Prop 3.7. (passo 7.) Seja $W$ uma janela de tamanho $2 \times 2$ de $A$, então count $W\left(W, P_{i}, f, A\right)$ $-\operatorname{count} W\left(W, P_{i T}, f, A\right)=\operatorname{count} B W\left(W, Q_{i}, A\right)$.

Demonstração. A prova é feita para o caso $i=3$. Outros casos podem ser provados de forma análoga. ${ }^{1}$ A prova para essa proposição foi feita em duas etapas.

- count $B W\left(W, Q_{3}, A\right)=0$

Se count $B W\left(W, Q_{3}, A\right)=0$ então ou $|A \cap W|<3$ ou $|A \cap W|=4$. Portanto, é verificado que:

- Se $|A \cap W|<3$, então para qualquer $W \in \mathcal{W}(A)$ na imagem $f$, a intersecção $W \cap A$ possui no máximo dois pixels $p, q$, tal que $f(p) \geq f(q)>f(r), \forall r \in W \backslash(W \cap A)$, isso implica que qualquer padrão $P$ contado nessa janela tenha pelo menos dois símbolos $L$ e/ou $l$, por inspeção é verificado que nenhum padrão em $P_{3}$ e $P_{3 T}$ possui essa propriedade, portanto, count $W\left(W, P_{3}, f, A\right)-\operatorname{count} W\left(W, P_{3 T}, f, A\right)=0$.

- Se $|A \cap W|=4$, então pode ocorrer de count $W\left(W, P_{3}, f, A\right)=1$, ou seja, existir $P \in$ $\mathcal{M} W_{P_{3}, f, A}(W)$, então, é preciso analisar todos os padrões que $P$ possa ser:

* Se $P \in\left\{\begin{array}{ll}G & 0 \\ L & G\end{array}\right],\left[\begin{array}{ll}o & g \\ L & G\end{array},\left[\begin{array}{ll}g & g \\ L & o\end{array}\right]\right\}$ então $b l \in(W \cap A)$ pela hipótese $|W \cap A|=4 \mathrm{e}$

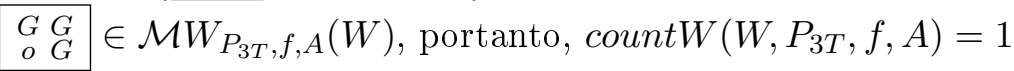

Os casos em que $P \in\left\{\begin{array}{ll}o & G \\ G & L\end{array},, \begin{array}{ll}g & o \\ G & L\end{array},, \begin{array}{ll}g & g \\ o & L\end{array}\right\}, P \in\left\{\begin{array}{ll}G & L \\ o & G\end{array},, \begin{array}{ll|l}G & L \\ g & o\end{array},, \begin{array}{ll}L & G \\ o & g\end{array}\right\}$ ou $P \in\left\{\begin{array}{ll}L & G \\ G & o\end{array}, \begin{array}{ll}L & G \\ o & g\end{array},, \begin{array}{ll}L & 0 \\ g & g\end{array}\right\}$ são análogos ao caso de exemplo acima. Portanto, se $|W \cap A|=$ 4 e count $W\left(W, P_{3}, f, A\right)=1$ então count $W\left(W, P_{3 T}, f, A\right)=1$ e count $W\left(W, P_{3}, f, A\right)-$ count $W\left(W, P_{3 T}, f, A\right)=0$, pela Prop. 3.5, se count $W\left(W, P_{3}, f, A\right)=0$ então

count $W\left(W, P_{3 T}, f, A\right)=0$, portanto, quando $|W \cap A|=4$ então count $W\left(W, P_{3}, f, A\right)-$ count $W\left(W, P_{3 T}, f, A\right)=0$.

Portanto, se count $B W\left(W, Q_{3}, A\right)=0$ então count $W\left(W, P_{3}, f, A\right)-\operatorname{count} W\left(W, P_{3 T}, f, A\right)=0$.

- count $B W\left(W, Q_{3}, A\right)=1$

Se count $B W\left(W, Q_{3}, A\right)=1$, então existe algum quad $Q \in M W_{Q_{3}, A}(W)$. Neste caso, é necessário analisar os possíveis valores de $Q$, um exemplo dessa análise é exibida abaixo:

- Se $\left.Q=\begin{array}{ll}1 & 1 \\ 0 & o\end{array}\right]$ e $\lambda=\min \{f(q): q \in(W \cap A)\}$ então pode ocorrer os seguintes casos.

$$
\begin{aligned}
& \text { * } \begin{array}{ll}
g & g \\
L & o
\end{array} \in \mathcal{M} W_{P_{3}, f, A}(W) \text {, se } f(b r)=\lambda \text {. } \\
& \text { * } \begin{array}{cc}
o & g \\
L & G
\end{array} \in \mathcal{M} W_{P_{3}, f, A}(W) \text {, se } f(t l)=\lambda \text { e } f(b r)>f(t l) \text {. } \\
& \text { * } \begin{array}{cc}
G & g \\
L & G
\end{array} \in \mathcal{M} W_{P_{3}, f, A}(W) \text {, se } f(t r)=\lambda, f(b r)>f(t r) \text { e } f(t l)>f(t r) \text {. }
\end{aligned}
$$

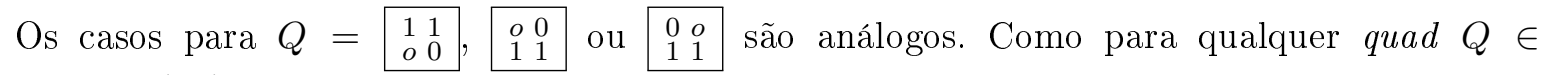
$M W_{P_{3}, A}(W)$, independente da combinação de níveis de cinza, também existe um padrão $P \in$ $\mathcal{M} W_{P_{3}, f, A}(W)$ logo é concluído que se count $B W\left(W, Q_{3}, A\right)=1$ então count $W\left(W, P_{3}, f, A\right)=$ 1.

\footnotetext{
${ }^{1}$ Uma prova computadorizada foi desenvolvida para essa proposição. Nessa prova, todas as janelas de tamanho $2 \times 2$ possíveis (considerando as relações de ordem entre os pixels) foram geradas e as funções countW e count $B W$ foram verificadas uma por uma. Mais detalhes da prova computadorizada e o seu código-fonte em linguagem Python estão disponíveis em http://www.vision.ime.usp.br/ dennis/hidden/proof/.
} 
Provando os casos acima, a condição "necessária" é provada por contra-posição. A Prop. 3.5 garante que $(i)$ count $W\left(W, P_{3}, f, A\right)-$ count $W\left(W, P_{3 T}, f, A\right)=1$ ou 0 e pela Prop. 3.1 é verdade que $\left(\right.$ ii) count $B W\left(W, Q_{3}, A\right)=1$ ou 0 . Desses resultados, é concluído que a negação de $(i)$ é dada por count $W\left(W, P_{3}, f, A\right)-\operatorname{count} W\left(W, P_{3 T}, f, A\right) \neq 1$ que implica em count $W\left(W, P_{3}, f, A\right)$ - count $W\left(W, P_{3 T}, f, A\right)=0$ e a negação de $(i i)$ é dada por count $B W\left(W, Q_{3}, A\right) \neq 1$ que implica em count $B W\left(W, Q_{3}, A\right)=0$. Portanto, a prova da condição "necessária" é dada por:

- $\operatorname{count} W\left(W, P_{3}, f, A\right)$ - count $W\left(W, P_{3 T}, f, A\right)=1 \Rightarrow$ count $B W\left(W, Q_{3}, A\right)=1$, utilizando contra-posição, isso é equivalente a count $B W\left(W, Q_{3}, A\right)=0 \Rightarrow \operatorname{count} W\left(W, P_{3}, f, A\right)-$ count $W\left(W, P_{3 T}, f, A\right)=0$ que foi provado acima.

- $\operatorname{count} W\left(W, P_{3}, f, A\right)-\operatorname{count} W\left(W, P_{3 T}, f, A\right)=0 \Rightarrow$ count $B W\left(W, Q_{3}, A\right)=0$, utilizando contra-posição, isso é equivalente a count $B W\left(W, Q_{3}, A\right)=1 \Rightarrow \operatorname{count} W\left(W, P_{3}, f, A\right)-$ count $W\left(W, P_{3 T}, f, A\right)=1$ que foi provado acima.

Prop 3.8. item 8. $\kappa W_{Q_{i}}(A)=\kappa W_{P_{i}}(A)$

Demonstração. Pela Prop. 3.7 é obtido a seguinte relação para uma dada janela $W \in \mathcal{W}(A)$ :

$$
\operatorname{count} B W(W, Q i, A)=\operatorname{count} W\left(W, P_{i}, f, A\right)-\operatorname{count} W\left(W, P_{i T}, f, A\right),
$$

somando esse resultado para cada janela de $\mathcal{W}(A)$, é obtido a seguinte equação:

$$
\sum_{W \in \mathcal{W}(A)} \operatorname{count} B W\left(W, Q_{3}, A\right)=\sum_{W \in \mathcal{W}(A)} \operatorname{count} W\left(W, P_{3}, f, A\right)-\operatorname{count} W\left(W, P_{3 T}, f, A\right),
$$

que demonstra a proposição.

A Prop. 3.2 garante que $\kappa_{Q_{i}}(A)=\kappa W_{Q_{i}}(A)$ e a Prop.3.6 garante que $\kappa_{P_{i}}(A)=\kappa W_{P_{i}}(A)$. Considerando esses resultados e a Prop. 3.8, é concluído que:

$$
\kappa_{Q_{i}}(A)=\kappa_{P_{i}}(A),
$$

Provando assim o Teo. 1. 


\section{Capítulo 4}

\section{Otimização por Árvore de Decisão}

Árvore de decisão é uma forma de classificar itens de maneira hierárquica de acordo com um conjunto de perguntas realizadas sobre esses itens. Os nós que não são folhas de uma árvore de decisão representam perguntas sobre os itens a serem classificados e as arestas que ligam um nó pai aos nós filhos representam as possíveis respostas para a pergunta representada pelo nó pai. Os nós folhas representam classes. A classificação de um dado item na árvore de decisão é dada pela folha que o item alcançar no percurso que corresponde as respostas das perguntas para esse item na árvore de decisão [KS08].

Suponha que dados os pontos $A, B, C$ de um triângulo e uma função $l$ que mede o comprimento de um segmento de reta entre dois pontos, é desejado classificar esse triângulo em equilátero, isósceles ou escaleno. Essa classificação pode ser modelada por uma árvore de decisão conforme exibido na Fig. 4.1

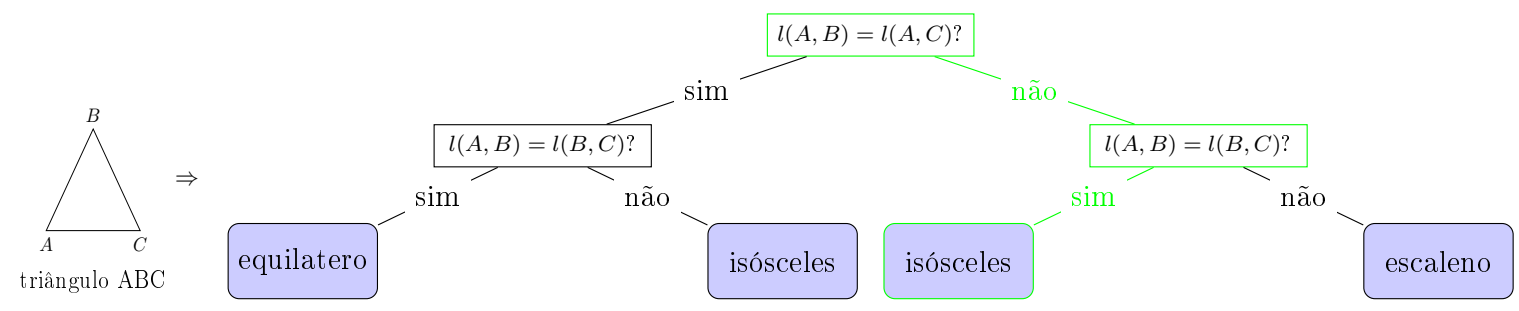

Fig. 4.1: Exemplo de árvore de decisão para classificação de um triângulo em equilátero, isósceles ou escaleno. As arestas em verde na árvore de decisão mostram o caminho percorrido para o triângulo $A B C$.

$\mathrm{Na}$ árvore de decisão da Fig. 4.1, primeiro são verificados se os segmentos $A B$ e $A C$ possuem o mesmo comprimento, se eles possuírem, então o próximo nó no caminho do triângulo na árvore de decisão classifica o triângulo como equilátero se os segmentos $A B$ e $A C$ também são iguais ou isósceles se são diferentes. Caso os comprimentos de $A B$ e $A C$ sejam diferentes, o próximo nó no caminho do triângulo na árvore de decisão classifica o triângulo como isósceles caso os segmentos $A B$ e $A C$ sejam iguais (e.g. triângulo $\mathrm{ABC}$ ) ou escaleno caso contrário.

Neste trabalho a árvore de decisão é utilizada para a otimização do número de comparações realizadas pela função count diminuindo o número de comparações realizadas de 180 (3 comparações para cada um dos 60 padrões) para 16.

\subsection{Otimização da Função count Utilizando Árvore de Decisão}

A função count definida na Sec. 3.1.2 realiza diversas comparações entre pixels de origem $(o)$ e vizinhanças definidas pelos domínios de todos os padrões de um conjunto $P_{i}$ definido por parâmetro. Além disso, o algoritmo de contagem de quads executa 9 vezes a função count (uma para cada conjunto de padrões: $\left.P_{1}, P_{2}, P_{3}, P_{4}, P_{D}, P_{1 T}, P_{2 T}, P_{3 T}, P_{D T}\right)$ para cada pixel da imagem.

A otimização realizada é baseada no fato de todos os pixels comparados pela função count estarem em uma vizinhança de 8-conectividade com o pixel de origem. Portanto, são definidos os 
pixels $v_{1}, v_{2}, v_{3}, v_{4}, v_{5}, v_{6}, v_{7}, v_{8} \in \mathcal{A}_{8}(p)$ como os pixels vizinhos de $p$ em $\mathcal{A}_{8}(p)$, conforme ilustrado pela Fig. 4.2 .

\begin{tabular}{|c|c|c|}
\hline$v_{1}$ & $v_{2}$ & $v_{3}$ \\
\hline$v_{4}$ & $p$ & $v_{5}$ \\
\hline$v_{6}$ & $v_{7}$ & $v_{8}$ \\
\hline
\end{tabular}

Fig. 4.2: Pixels vizinhos de um pixel $p$ em uma vizinhança de 8-conectividade.

A Fig. 4.2 exibe a posição relativa dos vizinhos de $p$ através das posições na grade, i.e. $v_{1}=$ $p+(-1,-1), v_{2}=p+(0,-1), \ldots, v_{8}=p+(1,1)$. Conhecendo a relação de ordem $(=,<,>)$ de todos os pixels dessa vizinhança com $p$, é possível determinar o resultado da função count para qualquer conjunto de padrões.

Para otimização da função count, é definido uma árvore de decisão, onde cada nó não folha é uma pergunta de qual relação de ordem os pixels em $\mathcal{A}_{8}(p)$ tem com $p$ e cada nó folha é uma função $n p: \mathbb{P} \rightarrow \mathbb{Z}$ que indica a contagem (função count) dos conjuntos de padrões para o pixel $p$. A Fig. 4.3 representa de forma resumida a árvore de decisão para a função count.

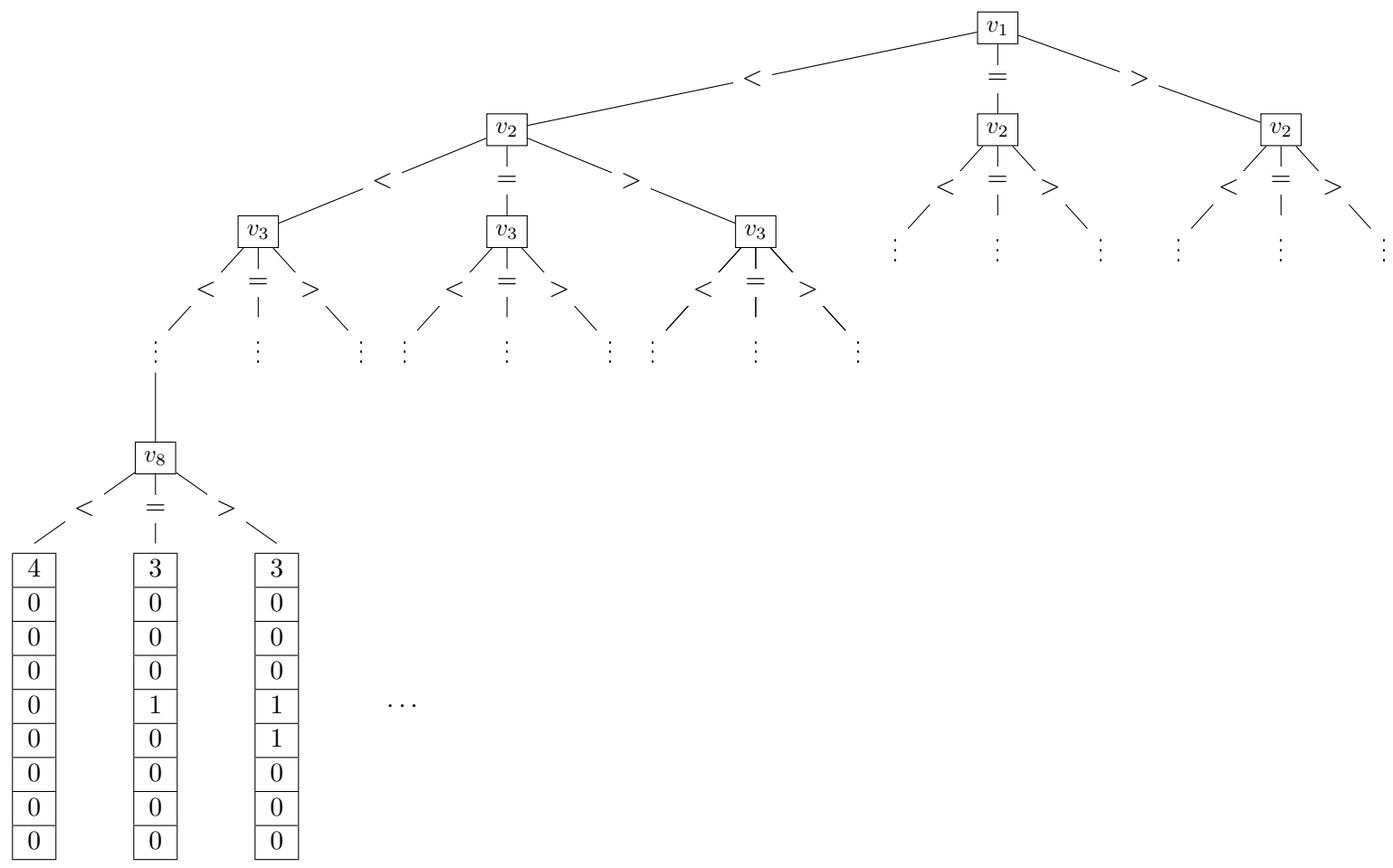

Fig. 4.3: Árvore de decisão resumida para a função count. Cada vértice representa um pixel vizinho de $p$, cada aresta representa a relação de ordem de $p$ com o vizinho e as folhas representam funções np onde cada valor é a quantidade de padrões de determinado tipo $\left(P_{1}, P_{2}, P_{3}, P_{4}, P_{D}, P_{1 T}, P_{2 T}, P_{3 T}, P_{D T}\right.$ respectivamente de cima para baixo na figura) contados por count.

Dado um pixel p em uma imagem $f$ qualquer, é possível utilizar a árvore apresentada na Fig. 4.3 para encontrar o valor da função count para qualquer conjunto de padrões. Cada vértice da árvore representa um pixel vizinho e cada aresta denota uma relação de ordem entre o pixel denotado pelo nó da árvore e o pixel $p$. Ao alcançar um nó folha em um percurso nessa árvore, é possível obter o valor de $\operatorname{count}\left(p, P_{i}, f\right)$ para qualquer $i \in\{1,2,3,4, D, 1 T, 2 T, 3 T, D T\}$ realizando apenas uma operação de consulta. 


\subsection{Implementação da Otimização Utilizando Árvore de Decisão}

O primeiro passo para a implementação da otimização proposta foi escolher a representação de matriz da árvore de decisão, onde o índice da coluna representa um caminho na árvore de decisão e todas linhas de uma coluna representam um nó folha (função $n p$ ), de tal maneira que cada linha é a contagem de um conjunto de padrões.

$\mathrm{Na}$ árvore de decisão proposta, cada nível representa a verificação da relação de ordem de um pixel $p$ com um dos seus 8 pixels vizinhos. Para cada nó de um determinado nível na árvore de decisão são adicionados 3 novos nós no próximo nível (um para cada tipo de relação: $<,=,>$ ). Logo a quantidade de nós em um determinado nível $l$ é dada por $3^{l}$, e consequentemente é concluído que a árvore de decisão proposta tem $3^{8}=6561$ nós folhas (um nível para cada pixel na vizinhança de 8-conectividade).

É possível representar a árvore de decisão com uma matriz de 6561 colunas e 9 linhas. O índice da coluna indica o caminho percorrido até o nó folha, para tal, cada possível caminho na árvore de decisão foi codificado em um número de base 3. Neste caso, cada tipo de aresta é representado por um dígito de base 3 , i.e. < é representado por $0,=$ é representado por 1 e $>$ é representado por 2. A posição do dígito no número de base 3 codificado indica o pixel (nível da árvore) comparado. Utilizando essa codificação é possível converter o número da base 3 para a base 10 e encontrar o índice de sua coluna na matriz que representa a árvore de decisão. A Fig. 4.4 exibe um exemplo de codificação de um caminho na árvore de decisão representada como matriz.

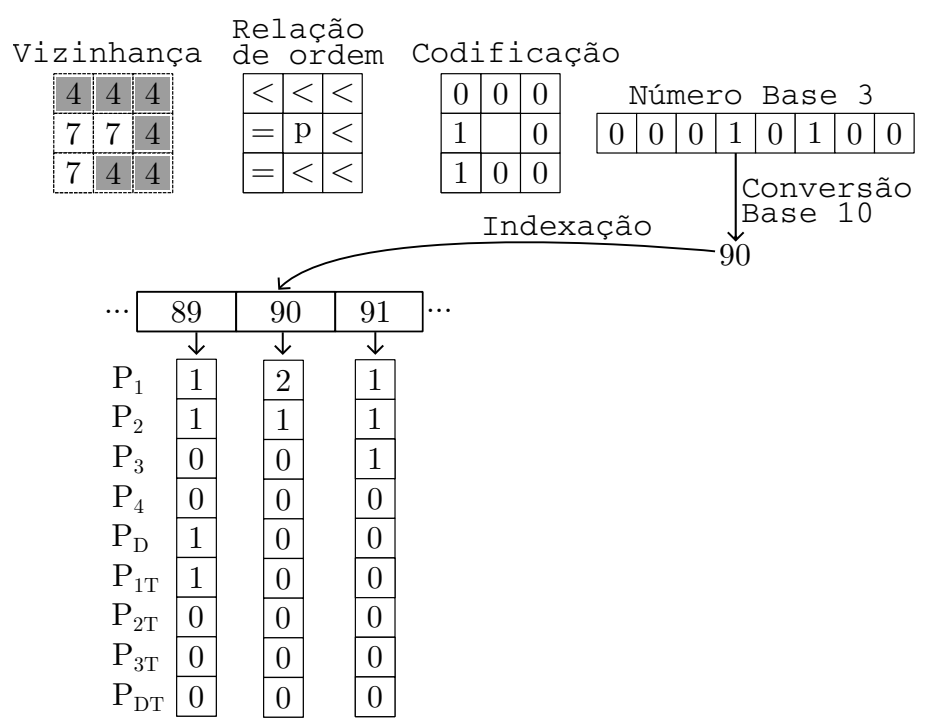

Fig. 4.4: Exemplo de codificação e indexação de uma vizinhança na matriz da árvore de decisão da função count.

A Fig. 4.4 exibe a vizinhança $\mathcal{A}_{8}(p)$ de um pixel $p$ e o processo de mapeamento na matriz da árvore de decisão. Esse processo se inicia verificando a relação de ordem de cada pixel vizinho de $p$ com o próprio $p$ e codificando essa relação em um dígito na base 3 , então o número formado por todos os dígitos codificados é convertido para a base 10 e utilizado como índice de coluna da matriz que representa a árvore de decisão.

Escolhido a representação de matriz para a árvore de decisão, foi necessário o desenvolvimento de um método para criar essa matriz. O método escolhido foi escrever um programa (em linguagem Python) para a geração de um arquivo que contém a matriz e ler esse arquivo na implementação do algoritmo de contagem de quads. O algoritmo implementado para a criação da matriz é apresentado no Alg. 12. 
Alg 12: Função Generate-Decision-Tree que cria a árvore de decisão da função count como uma matriz e grava essa matriz em um arquivo binário.

\section{Generate-Decision-Tree()}

1 let $S=\{0,1,2\}$

2 let $p=(1,1)$

3 foreach $N \in S^{8}$

$4 \quad i m g=$ CREATE-IMAGE $(p, N)$

$5 \quad$ index $=$ To-BASE10 $(N)$

$6 \quad$ foreach $P_{i} \in \mathbb{P}$

$7 \quad d t\left(\right.$ index,$\left.P_{i}\right)=\operatorname{count}\left(p, P_{i}, i m g\right)$

8 write $d t$ in a binary file.

O Alg. 12, inicia-se definindo o conjunto $S$ como os 3 símbolos de relação de ordem já codificados em dígitos de base 3 e o pixel p como o centro de uma vizinhança de 8-conectividade. Depois é tomado oito vezes o produto cartesiano de $S$ que representa todas as possíveis relações de ordem entre $p$ e seus oito vizinhos. Então para cada vizinhança com suas relações de ordem $N$, é gerado uma imagem img: $\{0,1,2\}^{2} \rightarrow S$ com o pixel $p$ no centro e as relações de ordem codificadas $N$ em seus vizinhos conforme exibido na Fig. 4.5.

\begin{tabular}{|c|c|c|}
\hline$N(0)$ & $N(1)$ & $N(2)$ \\
\hline$N(3)$ & 1 & $N(4)$ \\
\hline$N(5)$ & $N(6)$ & $N(7)$ \\
\hline
\end{tabular}

Fig. 4.5: imagem img gerada no Alg. 12

O Alg. 12 continua a criação da matriz calculando o índice da coluna através da conversão da codificação $N$ (número da base 3) em um número na base 10. Então para esse índice é armazenado a quantidade de cada conjunto de padrões calculada pela chamada da função count para a imagem img (note que os números utilizados na codificação da relação de ordem mantém a relação com a origem que tem valor 1, i.e. 0 que representa < é menor que 1,1 que representa $=$ é igual a 1 e 2 que representa > é maior que 1), portanto, a aplicação da função count na imagem img obtém a contagem desejada com referência as relações de ordem).

A última linha do algoritmo apenas grava um arquivo com os valores obtidos para todas as possíveis codificações em $S^{8}$, esses números podem ser representados em um byte (conjunto de 8 bits) já que o maior valor obtido para cada conjunto de padrões é 4 . Portanto, o arquivo gerado ao final do algoritmo contém 6561 conjuntos de 9 bytes, ou seja, o arquivo final tem $9 \times 6561=59049 \approx 59 k b$.

O segundo passo para a implementação da otimização foi integrar a árvore de decisão gerada (o arquivo binário) com o algoritmo de contagem de padrões. Essa integração é dada pela reescrita da função Pre-Process conforme mostrado no Alg. 13. 


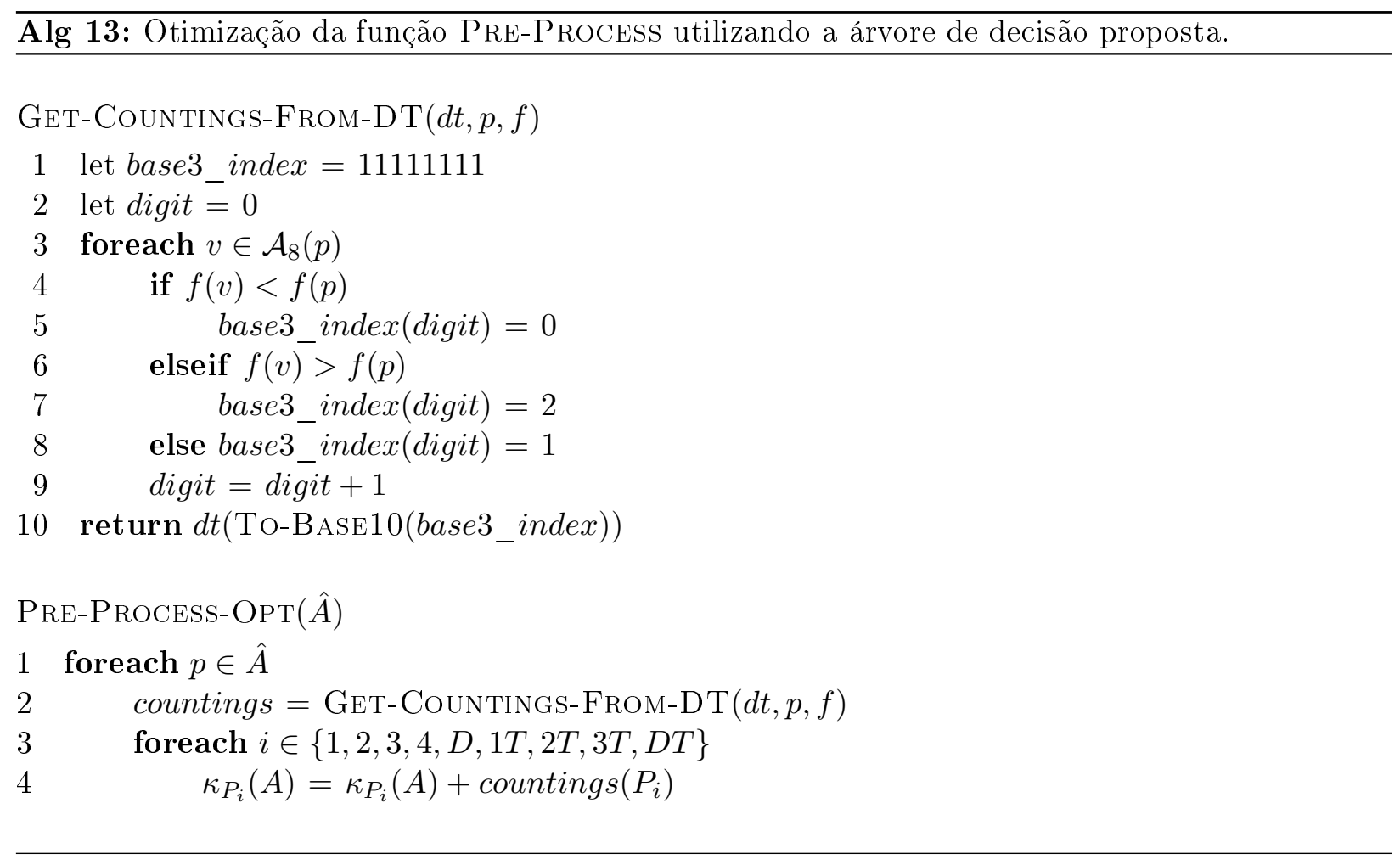

A função Pre-Process-Opt exibida no Alg. 13 é a versão otimizada da função Pre-Process do algoritmo de contagem de quads (Alg. 9 apresentado na Sec. 3.2). A versão otimizada considera que o arquivo que armazena a árvore de decisão já foi lido e disponibilizado pelo objeto $d t$. Nessa versão, a função count é substituída pelo procedimento GeT-CountingS-From-DT que calcula o caminho na árvore de decisão considerando o pixel analisado e sua vizinhança de 8-conectividade e devolve as contagens de todos os padrões para aquele pixel. O resultado da contagem para cada conjunto de padrões é feito através de acessos ao vetor countings retornado pela chamada a função Get-Countings-From-DT.

Considerando que o tempo gasto para ler o arquivo que contém a árvore de decisão codificada gasta tempo constante $R$ e a função de converter um número de base 3 para base 10 gasta tempo constante $C$, então é possível concluir que o algoritmo gasta tempo proporcional a $|\mathcal{D}| C+R$, logo, se $C$ é muito menor que $|\mathcal{D}|(C<<|\mathcal{D}|)$, que é verdadeiro para quase todas as imagens exceto para as imagens muito pequenas, o algoritmo gasta tempo linear no tamanho da imagem. Embora os dois algoritmos propostos apresentem tempo gasto linearmente proporcional ao tamanho da imagem original, o algoritmo otimizado realiza 16 comparações para cada pixel da imagem (2 comparações para cada pixel vizinho nas linhas 4 e 6) e o algoritmo não otimizado realiza 180 comparações por pixel da imagem (4 comparações por cada padrão dos 60 existentes). Mesmo considerando os tempos $C$ e $R$ gastos a mais na otimização, a Sec. 5.1 exibe que o algoritmo otimizado foi mais rápido em todas as imagens testadas. 


\section{Capítulo 5}

\section{Experimentos}

Implementações dos algoritmos propostos foram desenvolvidas em linguagem Java para a realização de experimentos práticos e a análise dos mesmos. Os métodos propostos utilizam a implementação da max-tree da biblioteca mmlib $4 j^{1}$. Apesar do algoritmo otimizado já estar implementado na biblioteca, os experimentos apresentados nessa seção não utilizam essa implementação para evitar que qualquer processamento extra que possa ocorrer altere os resultados dos experimentos.

Os experimentos comparam 3 métodos distintos que calculam os atributos: área, perímetro e número de Euler para todos os nós de uma árvore de componentes com os algoritmos propostos nesse trabalho. Os métodos a serem comparados são descritos abaixo:

- Métodos Incrementais (MI): Esse método junta três algoritmos incrementais diferentes para calcular os atributos: área, perímetro e número de Euler. Nesse método, em cada passo do algoritmo incremental (pré-processamento, intercalação e pós-processamento) são executados os respectivos passos dos algoritmos que calculam a área, perímetro e número de Euler. Com essa adaptação os três atributos são calculados realizando apenas um percurso na árvore de componentes. Os algoritmos utilizados foram:

- O algoritmo incremental apresentado na Sec. 2.2.2 para o cálculo da área.

- O algoritmo apresentado em [JWR04] para o cálculo do perímetro.

- Uma adaptação do algoritmo de Climent e Oliveira discutido na Sec. 2.3.2 para o cálculo do número de Euler.

- Chain Code: O método de chain code é um conhecido método que resulta no contorno dos componentes conexos de uma imagem binária. A implementação disponível no livro [BB16] foi adaptada para sua utilização na max-tree, de modo que a execução é realizada apenas dentro de uma janela que contém todos os pixels do componente conexo representado pelo nó. O algoritmo varre todos os nós da max-tree, executa o algoritmo de chain code em cada nó (apenas dentro da janela) e do contorno resultante extrai o valor de perímetro.

- Contagem de Quads Não Incremental (CQNI): Neste método os atributos são calculados através da contagem de quads em cada nó da árvore de componentes (sem a utilização de computação incremental). Neste caso, a contagem dos quads também é limitado pela janela que cobre o componente conexo para melhorar a eficiência do método.

Dois critérios foram utilizados na análise: $(i)$ tempo de execução e (ii) precisão do método em relação à aproximação do espaço contínuo do cálculo de perímetro e circularidade de círculos e elipses.

\footnotetext{
${ }^{1}$ Biblioteca com implementação em Java de diversas funcionalidades de processamento de imagens, incluindo muitos métodos de morfologia matemática como árvore de componentes. Disponível em https://github.com/ wonderalexandre/mmlib4j
} 


\subsection{Análise de Tempo}

Os experimentos para a análise de tempo foram realizados utilizando 17 imagens da base disponível em https://www.lrde.epita.fr/wiki/Olena/MaxtreeReview (a imagem "washingtonDC.pgm" é muito grande e o computador utilizado nos experimentos não teve memória suficiente para processála). Os experimentos foram conduzidos, tal que dada a max-tree calculada pela biblioteca mmlib $j j$ os seguintes passos eram executados: $(i)$ contagem de tempo era iniciada; (ii) os atributos eram calculados pelo método analisado, (iii) o cronômetro era pausado; (iv) o tempo gasto era calculado.

Os experimentos foram realizados em um computador com processador Intel(R) Core (TM) i5 $M 430$ de $2.27 G H z$ e $4 \mathrm{~GB}$ de memória (separados em dois pentes $D D R 3$ de $2 G B$ cada). O código foi executado pela JVM (Java Virtual Machine) versão 1.8.0_101 - b13 com os seguintes parâmetros: "-Xms2500m -Xmx2500m" para aumentar a quantidade de memória disponível para a JVM. Os gráficos plotados com os dados dos experimentos representam o tempo médio gasto em relação ao tamanho da imagem, ou seja, todas as imagens de mesmo tamanho foram agrupadas e a média do tempo gasto para execução dos experimentos para imagens dessa determinada dimensão foi calculada. Os experimentos que comparam as médias de tempo do algoritmo proposto e de sua versão com a otimização são apresentados como um gráfico na Fig. 5.1.

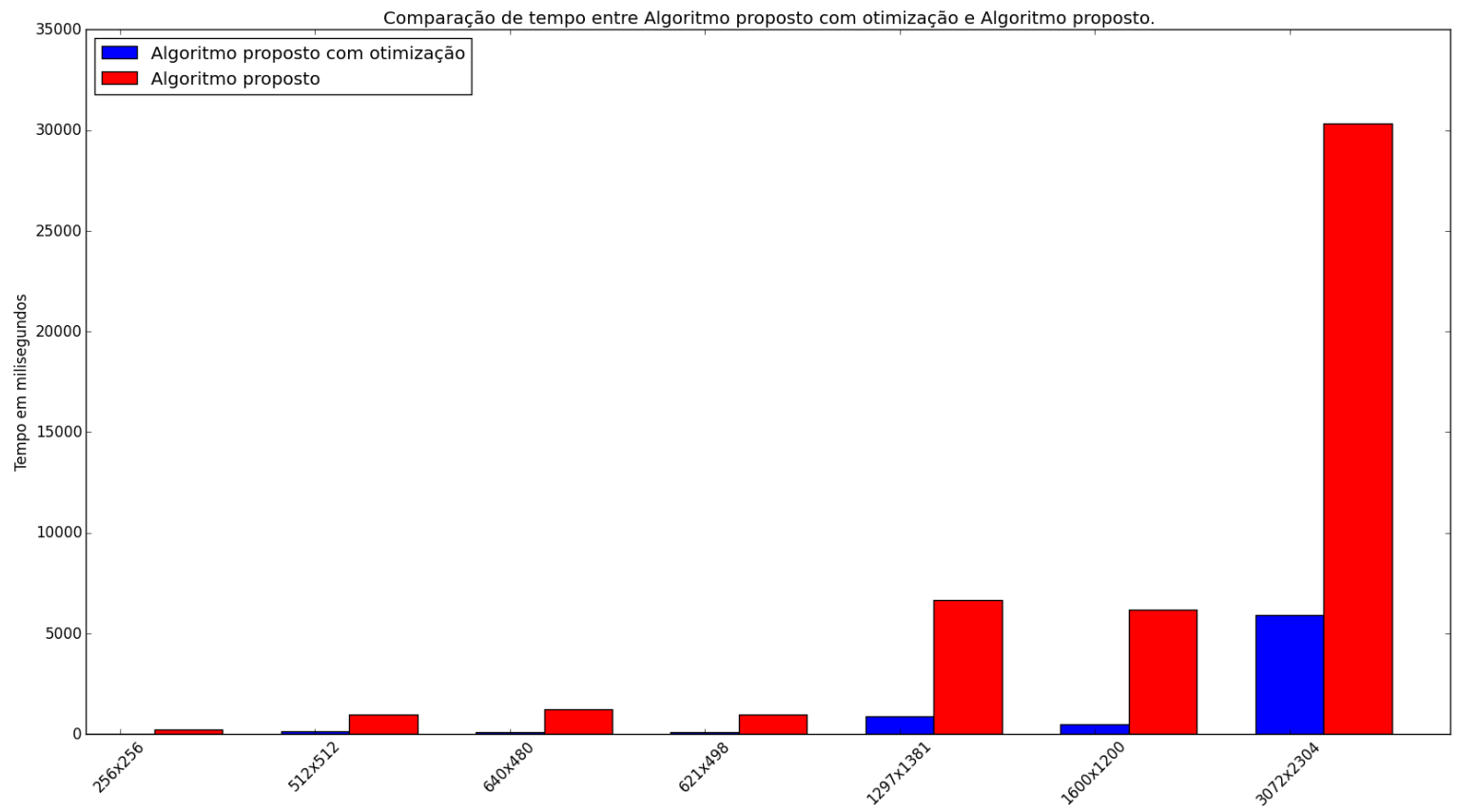

Fig. 5.1: Gráfico comparativo de tempo entre o algoritmo proposto e sua otimização.

Os experimentos mostram que o algoritmo proposto foi mais lento que sua versão otimizada em todas as dimensões avaliadas, permitindo assim concluir que os procedimentos de ler o arquivo da árvore de decisão e converter um número da base 3 para um número de base 10 para cada pixel são procedimentos com baixo custo computacional e que mesmo para imagens com dimensão $256 \times 256$ os custos dessas operações são mais baixos que o custo de executar 180 comparações por pixel executadas no algoritmo sem otimização. Os dados colhidos nos experimentos mostram que a versão otimizada do algoritmo foi em média aproximadamente 9,3 vezes mais rápida que a versão sem otimização.

A partir desse ponto, os resultados são comparados com a versão otimizada do algoritmo proposto, porque a mesma é a implementação mais competitiva do algoritmo apresentado nesse trabalho.

Os resultados dos experimentos que comparam o algoritmo proposto otimizado com $M I$ para o cálculo dos atributos são resumidos graficamente na Fig. 5.2. 


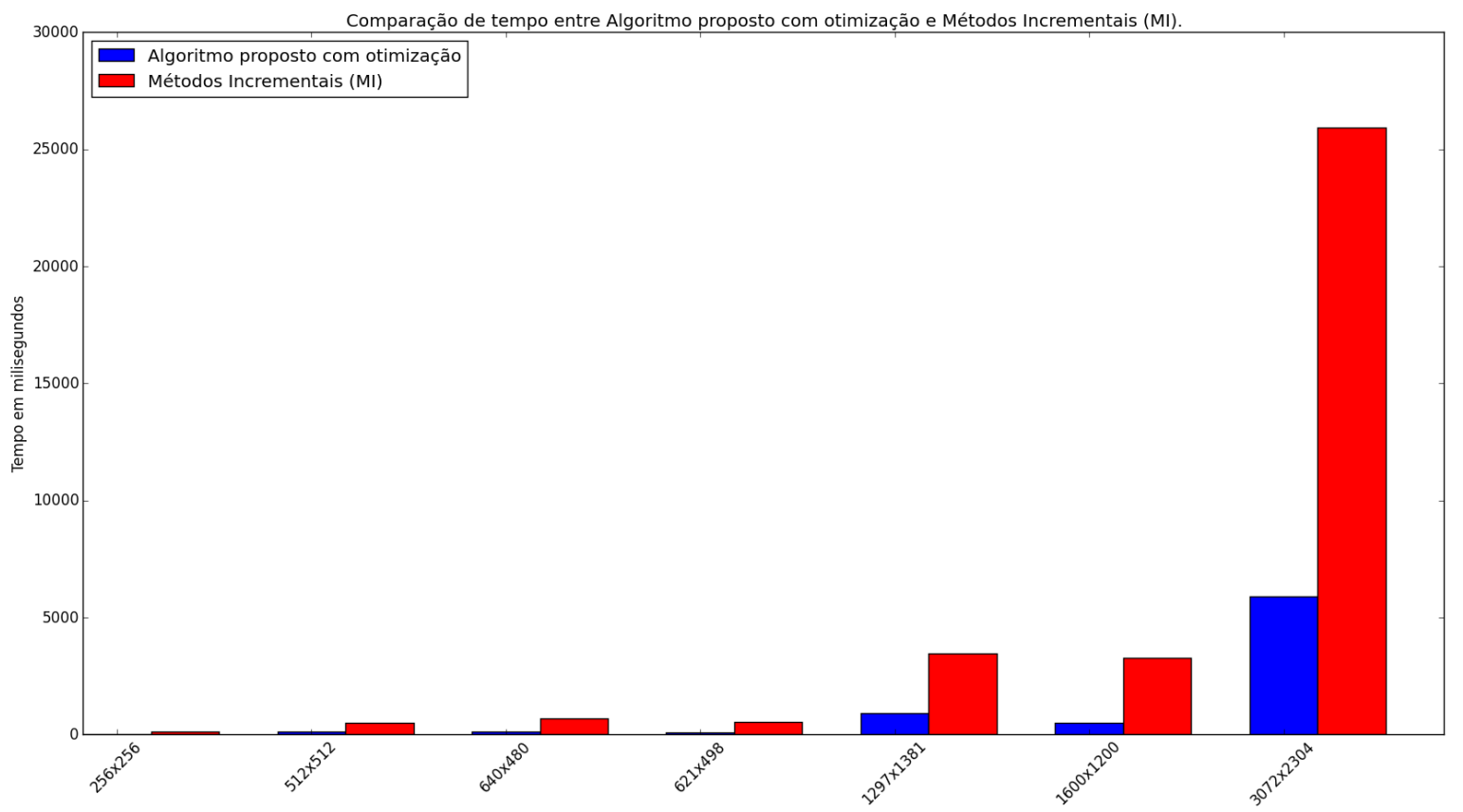

Fig. 5.2: Gráfico comparativo de tempo do algoritmo proposto otimizado e MI.

Os experimentos mostram que a implementação do algoritmo proposto com a otimização da árvore de decisão foi mais rápida que a junção dos métodos incrementais conhecidos para o cálculo dos atributos: área, perímetro e número de Euler, o speedup obtido foi de 5,3. Embora os métodos incrementais sejam muito eficientes para calcular um atributo individualmente, o seu desempenho pode se degradar ao calcular vários atributos de uma só vez. Isso não ocorre com o método proposto, porque todos os atributos podem ser extraídos das contagens de quads rapidamente, ou seja, calcular um atributo individualmente ou os possíveis atributos juntos não tem diferença significativa no tempo de execução.

A Fig. 5.3 exibe a plotagem dos dados comparativos de tempo entre a versão otimizada do algoritmo proposto e o algoritmo de chain code.

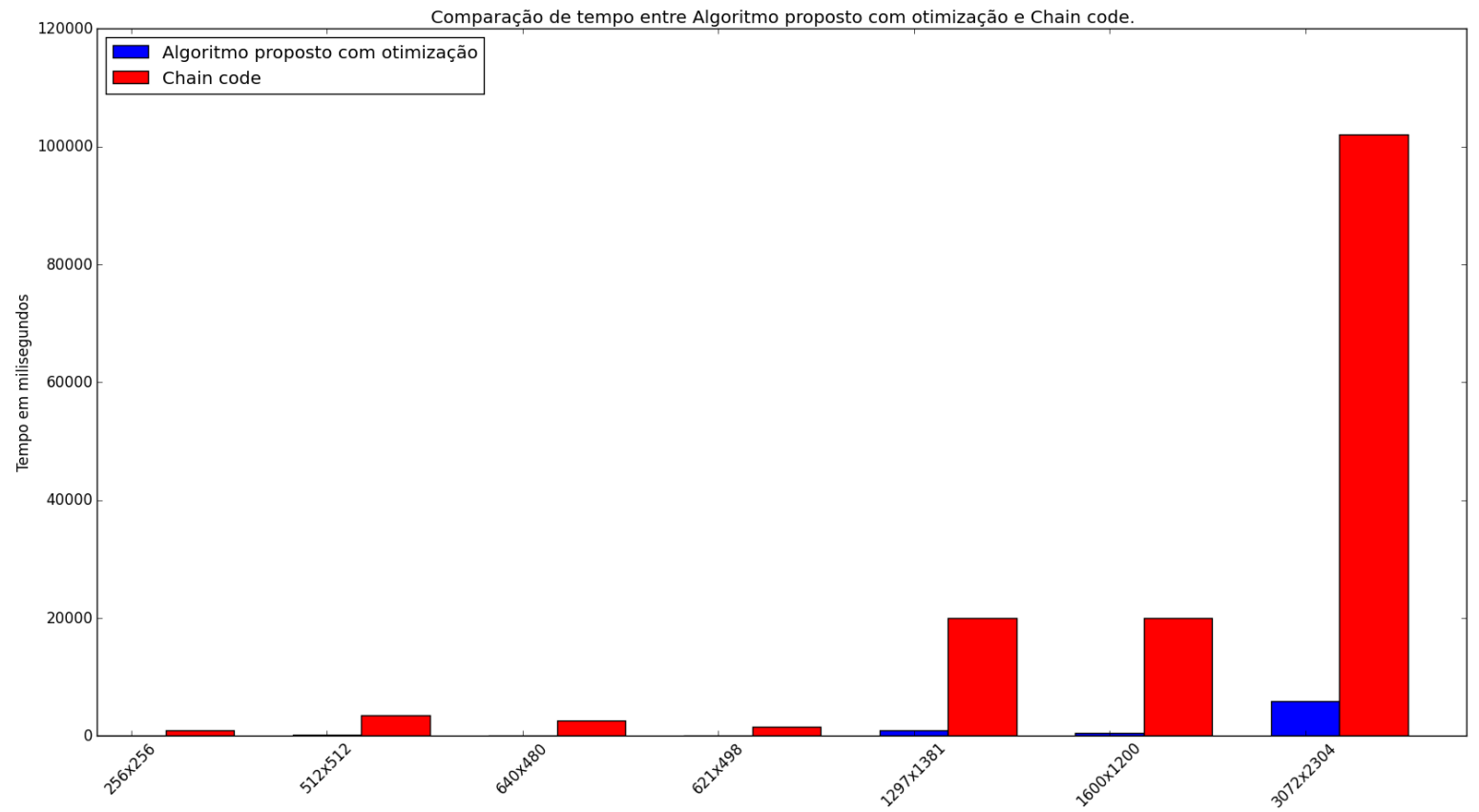

Fig. 5.3: Gráfico comparativo de tempo do algoritmo proposto otimizado e o algoritmo de chain code. 
O algoritmo de chain code extrai os contornos dos componentes conexos e calcula o perímetro por meio desses contornos. Embora flexível (pode calcular o perímetro considerando apenas borda interna, apenas borda externa ou ambas bordas) e preciso (conforme será exibido na Sec. 5.2), o mesmo é lento em relação à versão otimizada do algoritmo proposto que também possui uma aproximação ao espaço contínuo. Os experimentos mostram que o método otimizado para contar quads na árvore de componentes calculou o perímetro em média 26,9 vezes mais rápido que o algoritmo de chain code.

A comparação de tempo entre o algoritmo proposto otimizado e o método ingênuo de calcular os quads na árvore de componentes é exibida na Fig. 5.4.

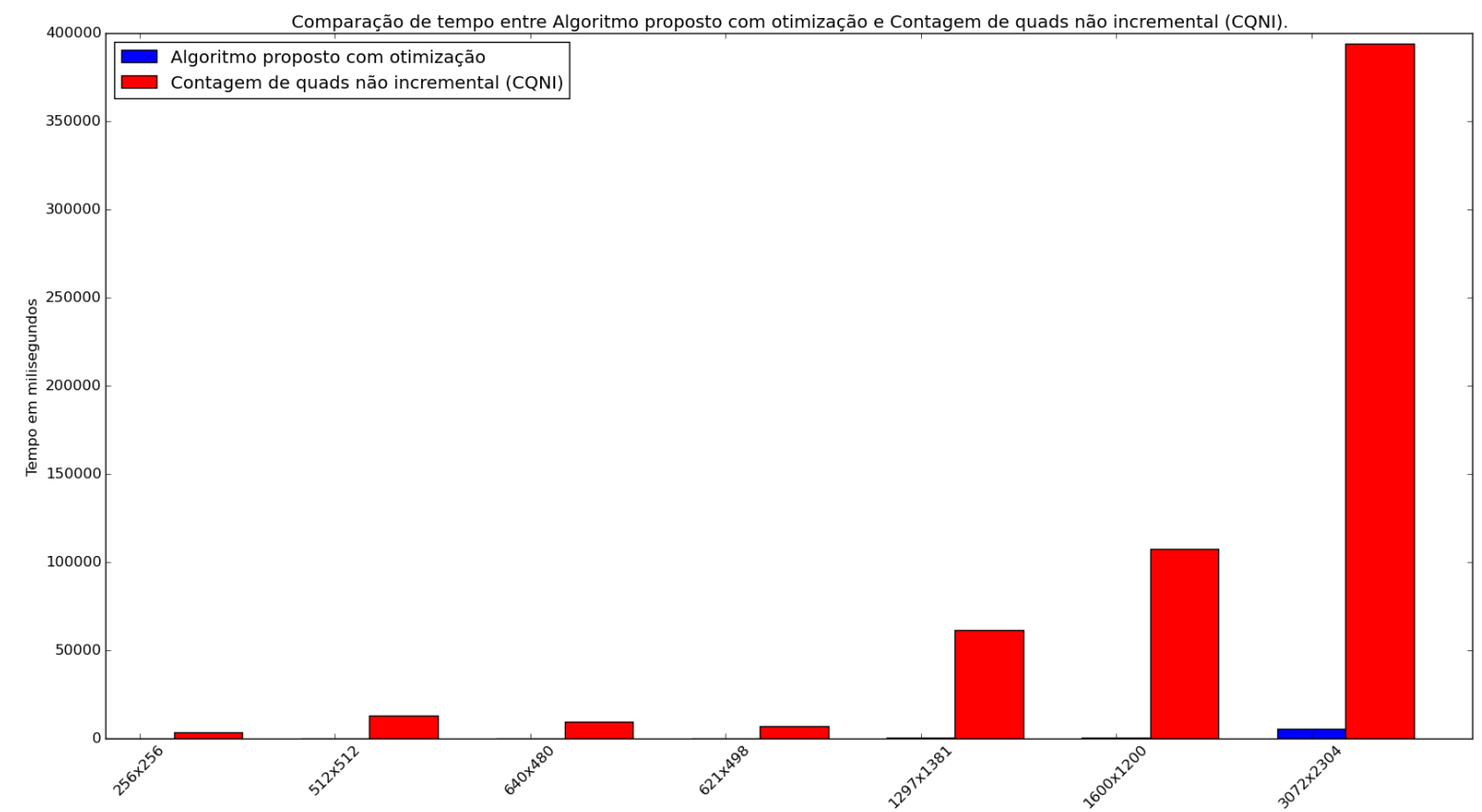

Fig. 5.4: Gráfico comparativo de tempo do algoritmo proposto otimizado e o algoritmo ingênuo de contagem de quads.

Os experimentos mostram que a contagem de quads de maneira não-incremental na árvore de componentes pode ser muito lenta em relação a sua implementação otimizada e incremental. O speedup neste caso foi de 111, 6 .

Os speedups do método proposto otimizado com os métodos analisados nos experimentos são exibidos na Tab.5.1.

\begin{tabular}{|c|c|c|c|c|}
\hline Dimensão & MI & CNBI & chain code & Algoritmo proposto sem otimização \\
\hline $256 \times 256$ & 6,023 & 150,759 & 39,281 & 9,668 \\
$512 \times 512$ & 3,956 & 104,559 & 27,696 & 7,842 \\
$640 \times 480$ & 6,383 & 91,297 & 24,599 & 11,528 \\
$621 \times 498$ & 6,022 & 77,490 & 16,078 & 10,819 \\
$1297 \times 1381$ & 3,858 & 68,530 & 22,271 & 7,427 \\
$1600 \times 1200$ & 6,763 & 222,010 & 41,148 & 12,762 \\
$3072 \times 2304$ & 4,386 & 66,665 & 17,260 & 5,131 \\
\hline Média & 5,342 & 111,616 & 26,905 & 9,311 \\
\hline
\end{tabular}

Tab. 5.1: speedup do algoritmo proposto otimizado em relação aos outros métodos analisados.

Conforme exibido pela Tab. 5.1, o algoritmo proposto otimizado foi o mais rápido entre todos os métodos analisados em todas as dimensões de imagem utilizadas nos experimentos. 


\subsection{Análise de Precisão}

Para a análise de precisão, é medido a proximidade que cada método consegue atingir do valor no espaço contínuo dos atributos: área, perímetro e circularidade de objetos em uma imagem sintética. A precisão discutida nesta seção refere-se a mesma discutida em [YALG94]. Neste sentido, é considerado que um objeto circular digitalizado é amostrado de um objeto circular com bordas arredondas e consequentemente um círculo tem valor de circularidade (relação entre perímetro e área) igual a 1. Dado um objeto digitalizado, é medindo qual método está mais próximo de alcançar os valores de atributo do objeto original amostrado. Como objetos retangulares geralmente possuem boas aproximações, independente do método utilizado, foram gerados apenas círculos e elipses para a análise neste caso. Os círculos foram aproximados utilizando a seguinte fórmula:

$$
x^{2}+y^{2} \leq r^{2},
$$

onde $r$ é o raio do círculo e $x$ e $y$ são coordenadas. Pela natureza discreta de imagens digitais as coordenadas $x$ e $y$ são valores inteiros e a imagem gerada é apenas uma aproximação do círculo com raio $r$. A elipse é aproximada pela seguinte fórmula:

$$
x^{2} h^{2}+y^{2} w^{2} \leq w^{2} h^{2},
$$

onde $x$ e $y$ são coordenadas discretas, $h$ é o comprimento do eixo vertical e $w$ o comprimento do eixo horizontal. A imagem gerada consiste em três círculos com raio 60, 120 e 200 pixels e três elipses com 300, 200 e 80 pixels de eixo vertical e 200, 150 e 120 pixels de eixo horizontal. A imagem gerada é exibida na Fig. 5.5

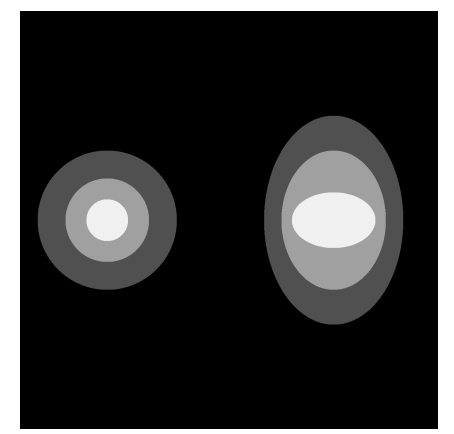

Fig. 5.5: Imagem utilizada para a análise de precisão.

Os valores esperados foram calculados utilizando as fórmulas analíticas para o cálculo de perímetro e de área de círculos conforme exibidas abaixo:

$$
\begin{gathered}
A_{C}=2 \pi r^{2} \\
P_{C}=2 \pi r,
\end{gathered}
$$

onde $P_{C}$ e $A_{C}$ são a área e o perímetro de um círculo respectivamente. O valor esperado para a área de cada elipse foi calculado utilizando a fórmula analítica e o perímetro foi utilizado a aproximação de Ramanujan, porque não existe uma fórmula analítica para o cálculo do perímetro de elipses. As fórmulas utilizadas para calcular a aproximação do perímetro e a área de elipses são exibidas abaixo:

$$
\begin{aligned}
& A_{E}=\pi a b \\
h= & \frac{(a-b)^{2}}{(a+b)^{2}} \quad P_{E} \approx \pi(a+b)\left(1+\frac{3 h}{10+\sqrt{4-3 h}}\right),
\end{aligned}
$$


onde $A_{E}$ e $P_{E}$ são área e o perímetro de uma elipse respectivamente, $a$ é o valor do maior eixo e $b$ o valor do menor eixo. Dados esses cálculos a medição de erro é feito de duas maneiras: $(i)$ calculando a distância do valor obtido pelo algoritmo utilizado ao valor desejado (calculado analiticamente) e (ii) porcentagem de erro [YALG94] dado por $E=100\left(\frac{\left|V_{E}-V_{A}\right|}{V_{E}}\right)$, onde $E$ é a percentagem de erro, $V_{A}$ o valor aproximado (calculado pelos algoritmos) e $V_{E}$ o valor exato (calculado analiticamente). A Tab. 5.2 exibe os valores calculados.

\begin{tabular}{|c|c|c|c|c|c|c|c|c|c|c|c|c|}
\hline & \multicolumn{6}{|c|}{ Métodos MI } & \multicolumn{6}{|c|}{ Algoritmo Proposto } \\
\hline & \multicolumn{2}{|c|}{ Área } & \multicolumn{2}{|c|}{ Perímetro } & \multicolumn{2}{|c|}{ Circularidade } & \multicolumn{2}{|c|}{ Área } & \multicolumn{2}{|c|}{ Perímetro } & \multicolumn{2}{|c|}{ Circularidade } \\
\hline & erro & \%erro & erro & $\%$ erro & erro & \%erro & erro & \%erro & erro & $\%$ erro & erro & \%erro \\
\hline & 44,559 & 0,023 & 429,204 & 27,3 & 0,367 & 38,3 & 24,941 & 0,013 & 102,34 & 6,5 & 0,113 & 11,8 \\
\hline & 36,706 & 0,029 & 343,363 & 27,3 & 0,383 & 38,3 & 21,794 & 0,017 & 68,043 & 5,4 & 0,099 & 9,9 \\
\hline & 36.78 & 0,039 & 300,443 & 27,3 & 0,375 & 38,3 & 12,72 & 0,013 & 67,300 & 6,1 & 0,109 & 11,1 \\
\hline & 15,934 & 0,035 & 206,018 & 27,3 & 0,383 & 38,3 & 19,066 & 0,042 & 40,826 & 5,4 & 0,099 & 9,9 \\
\hline & 24.289 & 0,080 & 171,681 & 27,3 & 0,368 & 38,3 & 3,211 & 0,010 & 41,636 & 6,6 & 0,115 & 12,0 \\
\hline & 22,733 & 0,201 & 103,009 & 27,3 & 0,384 & 38,4 & $-5,233$ & 0,046 & 19,827 & 5,3 & 0,097 & 9,7 \\
\hline Média & & 0,068 & & 27,3 & & 38,3 & & 0,023 & & 5,9 & & 10,8 \\
\hline
\end{tabular}

Tab. 5.2: Erros de área, perímetro e circularidade entre os MI e o algoritmo proposto.

A análise da Tab. 5.2 mostra que os algoritmos de contagem de quads tem uma aproximação melhor dos atributos perímetro e área e consequentemente a circularidade $\left(\frac{4 \pi A}{P^{2}}\right.$, onde $A$ é área do objeto analisado e $P$ é o perímetro). Porém, nota-se que a área possui aproximações com menos de $1 \%$ de erro em ambos os algoritmos por outro lado existe uma diferença significativa dos erros nos perímetros que impacta na circularidade. A análise de precisão do algoritmo de chain code é apresentado na Tab. 5.3.

\begin{tabular}{lll|ll}
\hline & \multicolumn{2}{c|}{ chain } & code & \multicolumn{2}{c}{ Algoritmo Proposto } \\
erro & \%erro & erro & \%erro \\
\hline & 15,991 & 1,01 & 102,335 & 6,5 \\
& 0,877 & 0,06 & 68,043 & 5,4 \\
& 6,270 & 0,57 & 67,300 & 6,1 \\
& 1,601 & 0,21 & 40,826 & 5,4 \\
& 2,700 & 0,86 & 41,636 & 6,6 \\
& 5,482 & 0,71 & 19,827 & 5,3 \\
Média & & 0,5 & & 5,9 \\
\hline
\end{tabular}

Tab. 5.3: Erro no cálculo de perímetro do algoritmo de chain code e do algoritmo proposto.

Como é possível notar na Tab. 5.3, o algoritmo de chain code consegue uma aproximação melhor que o algoritmo proposto, porém conforme mostrado na Sec. 5.1 ele é mais lento.

\subsection{Conclusão dos Experimentos}

Os experimentos mostraram que o algoritmo proposto com otimização têm bons resultados para o cálculo da área, perímetro e número de Euler na árvore de componentes. Esse algoritmo foi o mais rápido de todos os algoritmos testados, sendo em média 5,3 vezes mais rápido que o segundo algoritmo de maior desempenho (MI).

Apesar do algoritmo de chain code ter uma precisão maior que o algoritmo proposto para aproximações contínuas do perímetro, o mesmo foi em média 26, 9 vezes mais lento que algoritmo proposto. A contagem de quads na árvore de componentes pode calcular a aproximação contínua do perímetro com $5,9 \%$ de erro que é menor que os $27,7 \%$ obtido sem a realização da aproximação. Essa diferença é um fator importante para atributos que são compostos por outros atributos conforme mostrado com o exemplo da circularidade.

Portanto, é concluído que o algoritmo proposto com sua otimização é o mais adequado quando se deseja calcular os atributos: área, perímetro e número de Euler na árvore de componentes. O algoritmo proposto é o mais rápido e possui aproximações contínuas do perímetro com apenas $5,9 \%$ de erro, o que o torna um método interessante para calcular atributos compostos de área, perímetro e número de Euler na árvore de componentes. 


\section{Capítulo 6}

\section{Conclusões}

Neste trabalho foi apresentado o desenvolvimento de padrões baseados em bit-quads [Gra71] para o cálculo de atributos como: área, perímetro e o número de Euler para todos os nós de uma árvore de componentes de maneira incremental. Com esses padrões é possível calcular medidas com aproximação contínua que não é possível de calcular utilizando algoritmos incrementais conhecidos na literatura.

A otimização utilizando árvore de componentes apresentada no texto, conseguiu reduzir o tempo gasto para calcular a contagem dos chamados quads (uma adaptação equivalente aos bit-quads) em relação ao algoritmo proposto em [SAMH16]. O texto apresenta uma análise teórica que conclui assintoticamente que os algoritmos conhecidos na literatura e o algoritmo otimizado proposto gastam tempo linear no número de pixels, porém, a análise experimental mostrou que o algoritmo otimizado proposto é o mais rápido para calcular os três atributos calculáveis pelos bit-quads em conjunto.

O trabalho também enunciou o teorema que diz que a contagem dos quads nos nós da árvore de componentes é igual à contagem dos padrões propostos no trabalho e uma prova foi apresentada.

Em resumo, o trabalho apresenta um algoritmo eficiente para o cálculo de quads (bit-quads) na árvore de componentes e a partir dos quads extrai os atributos: área, perímetro e número de Euler em conjunto gastando menos tempo que os métodos conhecidos na literatura. O Algoritmo também é capaz de realizar aproximações contínuas do perímetro e da área que não são possíveis utilizando métodos incrementais conhecidos na literatura.

\subsection{Trabalhos Futuros}

Com a conclusão do desenvolvimento do algoritmo de contagem de quads (bit-quads) na árvore de componentes de maneira incremental apresentada nesse trabalho, os seguintes tópicos são sugeridos para estudos futuros:

- Adaptação dos padrões e/ou do algoritmo para serem utilizados na árvore de formas [GCCN13].

- Relacionar os padrões com o operador morfológico Hit-Miss [DL03], e se possível desenvolver um método que transforme uma imagem binária (elemento estruturante de composição) em um conjunto de padrões para serem utilizados de forma incremental na árvore de componentes.

- Estudar a aplicabilidade do algoritmo na árvore de partição binária [SW09]. 


\section{Referências Bibliográficas}

[AMCH13] W. A. L. Alves, A. Morimitsu, J. S. Castro e R. F. Hashimoto. Extraction of numerical residues in families of levelings. Em 2013 XXVI Conference on Graphics, Patterns and Images, páginas 349-356, Aug 2013. 3

[AMH15] W. A. L. Alves, A. Morimitsu e R. F. Hashimoto. Scale-space representation based on levelings through hierarchies of level sets. Em Proceedings of the 12th International Symposium on Mathematical Morphology and its Applications to Image and Signal Processing, ISMM '15, 2015. 3

[BB16] W. Burger e M.J. Burge. Digital Image Processing: An Algorithmic Introduction Using Java. Texts in Computer Science. Springer London, 2016. 51

$\left[\mathrm{BGL}^{+} 07\right]$ C. Berger, T. Geraud, R. Levillain, N. Widynski, A. Baillard e E. Bertin. Effective component tree computation with application to pattern recognition in astronomical imaging. Em 2007 IEEE International Conference on Image Processing, volume 4, páginas IV - 41-IV - 44, Sept 2007. 1

[BJ96] E. J. Breen e R. Jones. Attribute openings, thinnings, and granulometries. Computer Vision and Image Understandin, 64(3):377 - 389, 1996. 4

[CO14] J. Climent e L. S. Oliveira. A new algorithm for number of holes attribute filtering of grey-level images. Pattern Recognition Letters, 53:24-30, 2014. xv, 2, 3, 4, 5, 24, 25, $26,28,29,31,32,35$

[com16] The Scipy community. scipy.misc.imread - scipy v0.17.1 reference guide. http://docs. scipy.org/doc/scipy/reference/generated/scipy.misc.imread.html, 2016. acessado em 1507-2016. 1

[DL03] E. R. Dougherty e R. A. Lotufo. Hands-On Morphological Image Processing. SPIE, Junho 2003. 1, 57

[FSL04] A. X. Falcão, J. Stolfi e R. A. Lotufo. The Image Foresting Transform: Theory, Algorithms, and Applications. IEEE Transactions on Pattern Analysis and Machine Intelligence, 26(1):19-29, 2004. 1

[GCCN13] T. Géraud, E. Carlinet, S. Crozet e L. Najman. A quasi-linear algorithm to compute the tree of shapes of n-D images. Em R. Strand C.L. Luengo Hendriks, G. Borgefors, editor, International Symposium on Mathematical Morphology, volume 7883 of Lecture Notes in Computer Science, páginas 97-108, Uppsala, Sweden, Maio 2013. Springer. 57

[Gra71] S. B. Gray. Local properties of binary images in two dimensions. IEEE Transactions on Computers, C-20(5):551-561, May 1971. xiv, 2, 4, 20, 21, 22, 23, 24, 29, 31, 35, 57

[HM11] J. Hernandez e B. Marcotegui. Shape ultimate attribute opening. Image and Vision Computing, 29(8):533 - 545, 2011. 3 
[JWR04] A. C. Jalba, M. H. F. Wilkinson e J. B. T. M. Roerdink. Morphological hat-transform scale spaces and their use in pattern classification. Pattern Recognition, 37(5):901 915, 2004. 51

[KS08] C. Kingsford e S. L. Salzberg. What are decision trees? Nature biotechnology, 26(9):1011-1013, 2008. 45

[LMG05] M. León, S. Mallo e A. Gasull. A tree structured-based caption text detection approach. Em In Fifth IASTED VIIP. Citeseer, 2005. 3, 4, 5

[MAH15] A. Morimitsu, W. A. L. Alves e R. F. Hashimoto. Incremental and efficient computation of families of component trees. Em International Symposium on Mathematical Morphology and Its Applications to Signal and Image Processing, páginas 681-692. Springer, 2015. 9

[Mat16] MathWorks. Read image from graphics file - matlab imread. http://www.mathworks. $\mathrm{com} / \mathrm{help} / \mathrm{matlab} / \mathrm{ref} / \mathrm{imread} . \mathrm{html}$ ?s_tid=gn_loc_drop, 2016. acessado em 15-072016. 1

[NC06] L. Najman e M. Couprie. Building the component tree in quasi-linear time. IEEE Transactions on Image Processing, 15(11):3531-3539, Nov 2006. 1, 4

[Ope15] OpenCV. Opencv: Image file reading and writing. http://docs.opencv.org/3.1.0/d4/ da8/group__imgcodecs.html, 2015. acessado em 15-07-2016. 1

[SAMH16] D. J. Silva, W. A. L. Alves, A. Morimitsu e R. F. Hashimoto. Efficient incremental computation of attributes based on locally countable patterns in component trees. Em 2016 IEEE International Conference on Image Processing (ICIP), páginas 3738-3742, Sept 2016. 3, 4, 5, 57

[SG00] P. Salembier e L. Garrido. Connected operators based on region-tree pruning strategies. Em Proceedings 15th International Conference on Pattern Recognition. ICPR-2000, volume 3, páginas 367-370 vol.3, 2000. 4

[SOG98] P. Salembier, A. Oliveras e L. Garrido. Antiextensive connected operators for image and sequence processing. IEEE Transactions on Image Processing, 7(4):555-570, Apr 1998. 26

[SW09] P. Salembier e M. H. F. Wilkinson. Connected operators. IEEE Signal Processing Magazine, 26(6):136-157, November 2009. 1, 4, 9, 57

[TSR $\left.{ }^{+} 15\right]$ L. A. Tavares, R. M. Souza, L. Rittner, R. C. Machado e R. A. Lotufo. Interactive maxtree visualization tool for image processing and analysis. Em Image Processing Theory, Tools and Applications (IPTA), 2015 International Conference on, páginas 119-124, Nov 2015. 1

[URW07] E. R. Urbach, J. B. T. M. Roerdink e M. H. F. Wilkinson. Connected shape-size pattern spectra for rotation and scale-invariant classification of gray-scale images. Pattern Analysis and Machine Intelligence, IEEE Transactions on, 29(2):272-285, Feb 2007. 1

[Vin93] L. Vincent. Grayscale area openings and closings, their efficient implementation and applications. Em First Workshop on Mathematical Morphology and its Applications to Signal Processing, páginas 22-27, 1993. 4

[YALG94] L. Yang, F. Albregtsen, T. Lgnnestad e P. Grgttum. Methods to estimate areas and perimeters of blob-like objects: a comparison. Em In Proc. IAPR Workshop on Machine Vision Applications, pp.272276, páginas 27227-6, 1994. 55, 56 


\section{Índice Remissivo}

4-conectividade, 9

8-conectividade, 9

count, 32

count $B, 30$

count $B W, 38$

count $W, 39$

área, 23

árvore compacta e não redundante, 15

árvore de componentes, 13

árvore de decisão, 45

árvore de formas, 57

árvore de partição binária, 57

Chain Code, 51

Hit-Miss, 57

Python, 47

bit-quads, 22

cell-bit, 22

cell-group, 22

max-tree, 15

min-tree, 15

pixel, 7

quads, 24

speedup, 53

altitude, 14

altura de um nó na árvore, 14

aninhamento de conjuntos de níveis, 11

aproximação contínua, 23

arestas, 8

atributo, 16

atributo incremental, 17

círculos, 55

caminho, 8

circularidade, 55

componente conexo, 8

comprimento de caminho, 8

computação incremental, 16

conjunto cruz, 9

conjunto de matching em uma janela, 38

conjunto de componentes conexos, 10

conjunto de matching de padrões, 32

conjunto de matching de quads, 30 conjunto de nível, 11

conjunto de nível inferior de valor $\lambda, 11$

conjunto de nível superior de valor $\lambda, 11$

conjunto potência, 8

conjunto quadrado, 9

Contagem de quads não Incremental (CQNI), 51

corretude, 38

distância entre dois vértices, 8

elipses, 55

erro, 56

experimentos, 52

grafo, 8

grafo com pesos nos vértices, 9

grafo conexo, 8

imagem, 7

imagem multibanda, 7

intensidade associada, 14

janela de tamanho $2 \times 2,38$

limiarização, 11

localização de texto, 4

máximo local, 14

Métodos Incrementais (MI), 51

mínimo local, 14

menor componente conexo, 15

mosaico, 22

nó ancestral, 14

nó descendente, 14

nó filho, 14

nó pai, 14

nó raiz, 14

nós associados, 15

nós folhas, 14

número de base 3,47

número de Euler, 21

nível, 9

O conjunto de matching de padrões dentro de uma janela, 39 
padrões, 25

perímetro, 23

percurso de pós-ordem, 16

peso, 9

precisão do método, 51

prova computadorizada, 43

segmentação de aspirinas, 3

segmentação de placas, 3

tempo de execução, 51

vértices, 8

valor de limiar, 11

vizinho, 8 